\title{
A novel role for aconitase during erythroid development
}

\author{
Anne-Laure Talbot \\ Paris, France \\ BA, University of California at Berkeley, 2004 \\ MS, University of Virginia, 2009
}

\begin{abstract}
A Dissertation presented to the Graduate Faculty of the University of Virginia in Candidacy for the Degree of

Doctor of Philosophy
\end{abstract}

Department of Pathology

University of Virginia

December, 2011

Adam Goldfarb, M.D.

James Mandell, M.D., Ph.D.

Prabakhara Reddi, Ph.D

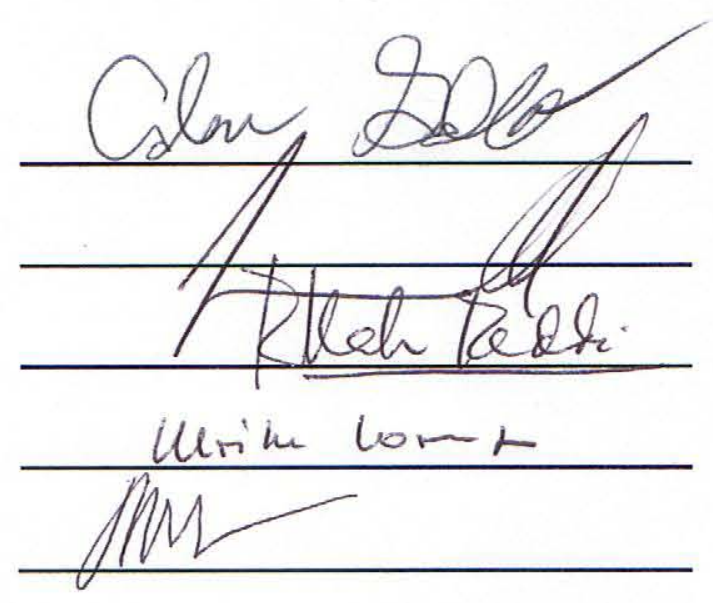

Ulrike Lorenz, Ph.D.

John Densmore, M.D., Ph.D. 


\section{ABSTRACT}

Erythropoiesis is the biological process by which red blood cells are produced from multipotent progenitors in the adult bone marrow. Due to their short 120 day lifespan, there is a constant demand for new red cells placed on the marrow. Proliferation, differentiation and survival of erythroid progenitors are tightly controlled by multiple factors, including levels of the hormone erythropoietin (EPO) and iron bioavailability. While abundant data indicate that EPO and iron cooperate to optimize red cell production, the precise mechanisms coupling these two factors remain poorly understood. Prior studies identified the aconitase enzymes as mediators of the erythroid response to iron deficiency. In the current study, a role for aconitase during erythropoiesis was evaluated in iron-replete conditions.

Based on a targeted pharmacological approach, results presented here demonstrate that aconitase activity regulates erythroid differentiation. Inhibition of aconitase with fluoroacetate (FA) impaired the proliferation and differentiation of primary human erythroid progenitors, but not viability or total ATP content. In vivo, aconitase inhibition caused a normochromic, normocytic anemia with low reticulocyte counts and elevated serum EPO, but spared other lineages. FA treatment also reduced the red cell mass in a mouse model of erythrocytosis caused by mutant JAK2V617F. A likely mechanism involves disruption of a subset of ERK1/2-mediated signaling events. Activation of the downstream ERK1/2 target RSK was severely diminished under aconitase inhibition, and inhibition of ERK activation recapitulated all the effects of the aconitase inhibitor. Furthermore, aconitase was found to physically interact with ERK1 and ERK2. 
Aconitase may act as a scaffold for ERK1/2 activation and target the kinase to key downstream effectors of the erythroid development program. Interestingly, ERK1 -/mice were partially resistant to the effects of the aconitase inhibitor, supporting a role for ERK1 in mediating the effects of aconitase inhibition. Taken together, these results support a regulatory role for aconitase in erythropoiesis, possibly as an integrator of EPO and iron-mediated signals. Targeting this pathway may yield novel therapeutic options in several diseases of dysregulated erythroid production. 


\section{TABLE OF CONTENTS}

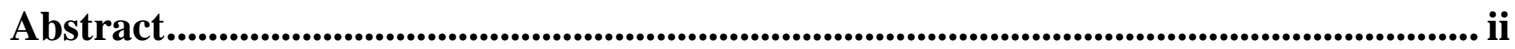

List of figures..................................................................................................... vii

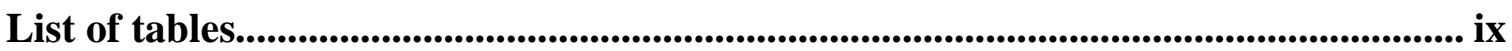

List of abbreviations ...................................................................................................................

Acknowledgements and dedication ........................................................................... xiv

CHAPTER 1: General Introduction ..........................................................................................1

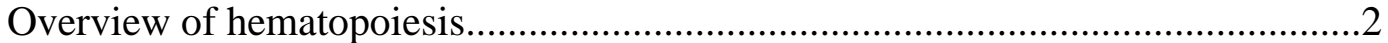

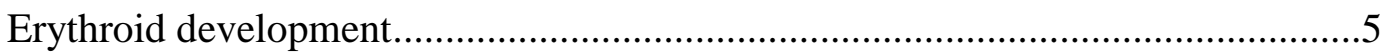

Commitment to the erythroid lineage ……………………………………....5

Erythroid developmental stages ................................................................6

The erythroblastic island ......................................................................10

Regulation of erythropoiesis ...........................................................................11

EPO-EPOR signaling ............................................................................11

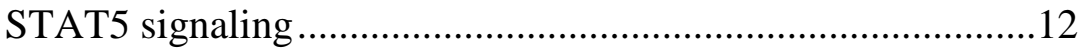

ERK signaling ........................................................................

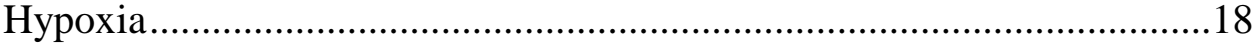

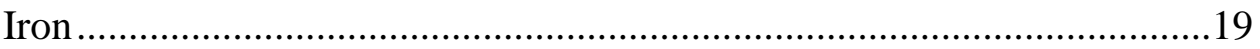

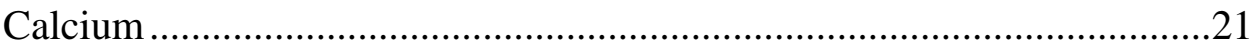

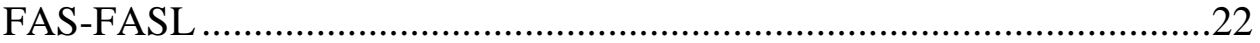

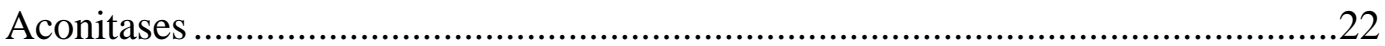

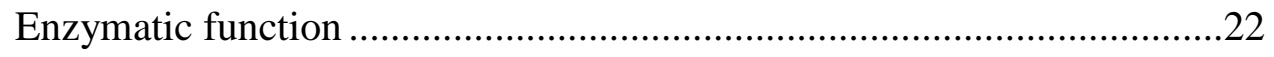

Regulation of catalytic activity ………………………………………....23

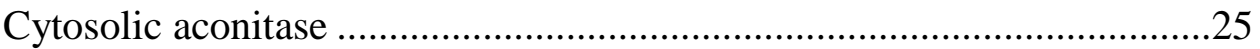

Mitochondrial aconitase .....................................................................28

Aconitases in erythropoiesis ..................................................................29

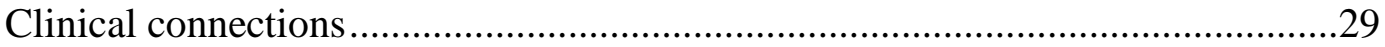

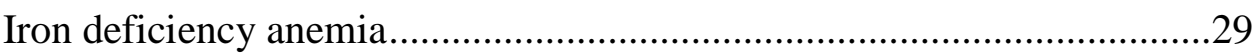


Polycythemia vera ..................................................................... 31

Clinical evidence for synergy between iron and EPO ............................34

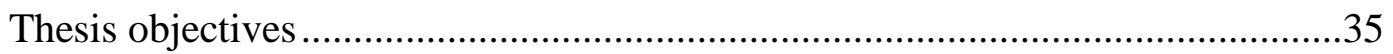

\section{CHAPTER 2: Aconitase Regulation of Erythropoiesis Correlates with a Novel}

Licensing Function in Erythropoietin-induced ERK Signaling .................................. 37

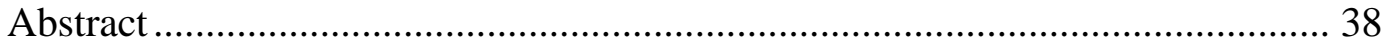

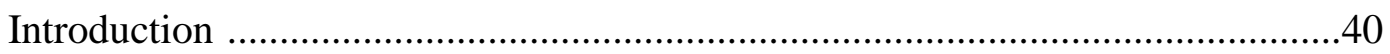

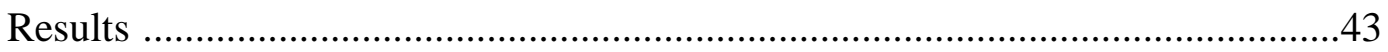

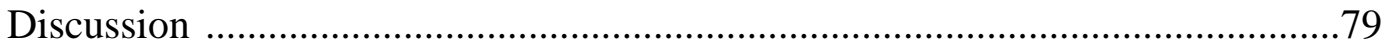

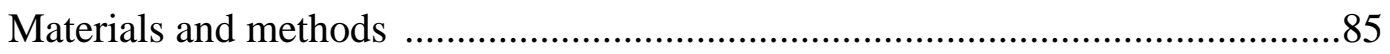

CHAPTER 3: Related Data ................................................................................................ 91

Are RSKs mediating the effects of aconitase on erythropoiesis? ......................92

Aconitase blockade impairs ERK-mediated activation of MNK ......................104

MEK activation is not dynamically regulated by EPO ...................................107

Elk-1 activation is not dynamically regulated by EPO ................................110

Deregulated ERK signaling is not caused by defects in

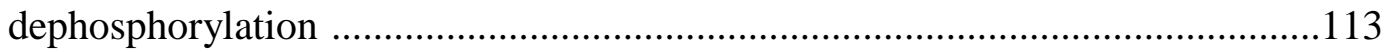

Aconitase inhibition correlates with increased ERK1/2 in an insoluble cellular

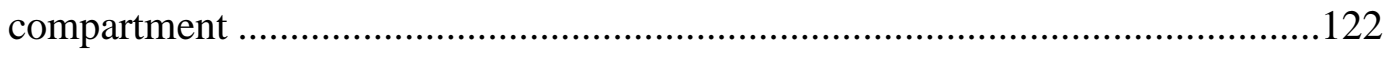

EPO does not promote ERK2 dimer formation ...........................................126

A role for ERK1 in mediating the effects of FA in vivo .................................129

Isocitrate does not rescue erythropoietic defects due to FA ...........................136

Materials and methods ..........................................................................141

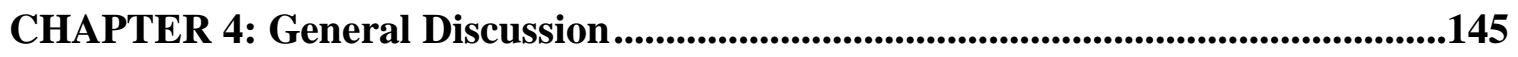

Aconitase activity regulates erythropoiesis .............................................146

Inhibition of aconitase leads to decreased red cell production ................146 
The erythroid lineage is highly sensitive to changes in aconitase activity 148

Iron deficiency and FA blockade of aconitase. 149

A role for ERK signaling during erythropoiesis 151

Inhibition of ERK activation impairs erythropoiesis in vitro 151

Signaling downstream of ERK1/2 155

Evidence for MEK independent activation of ERK 159

Mechanistic insights. 163

A novel aconitase-ERK interaction 163

Subcellular site of ERK activation: location matters 166

A mitochondrial signaling complex? 167

ERK1 vs. ERK2 171

A role for aconitase in unexplained anemia of the elderly?. .171

Clinical perspectives

Targeting aconitase in polycythemia vera 173

Augmenting or preserving erythroid-specific ERK signaling to treat

IDA

Summary of future directions 175

CHAPTER 5: References 


\section{LIST OF FIGURES}

\section{CHAPTER 1}

Figure 1.1 Current and alternative models for hematopoietic stem cell and blood lineage commitment

Figure 1.2 The erythroid differentiation pathway ………...........................................

Figure 1.3 Schematic representation of the ERK signaling cascade ...............................17

Figure 1.4 Iron-dependent regulation of IRP binding activity .......................................27

\section{CHAPTER 2}

Figure 2.1 Elevated serum citrate levels in mice treated with fluoroacetate .47

Figure 2.2 In vivo effects of aconitase

Figure 2.3 Analysis of the bone marrow erythroid maturation using Ter119 and CD44 51

Figure 2.4 Inhibition of aconitase impairs responsiveness to anemic challenge .54

Figure 2.5 Effects of aconitase inhibition on erythroid proliferation, survival and maturation in vitro.

Figure 2.6 FA inhibits erythropoiesis in the absence of changes in cellular ATP, ROS, or MMP

Figure 2.7 Aconitase inhibition interferes with EPO activation of ERK signaling in a pathway-specific manner

Figure 2.8 The effects of MEK inhibition on erythropoiesis recapitulate those of aconitase inhibition

Figure 2.9 Identification of an ERK-aconitase protein complex that is disrupted by aconitase inhibition

Figure 2.10 Aconitase inhibition does not prevent ERK dimerization.

Figure 2.11 Aconitase inhibition normalizes red cell counts in a murine model of polycythemia vera

Figure 2.12 Effects of FA and EPO on Ba/F3 cells expressing EPOR or EPOR JAK2V617F

Figure 2.13 Mice lacking cytosolic aconitase respond normally to FA infusion 


\section{CHAPTER 3}

Figure 3.1 Effects of RSK inhibition on erythroid maturation and proliferation resemble those of aconitase inhibition .94

Figure 3.2 Bi-D1870-mediated inhibition of RSK does not impair erythroid differentiation or proliferation

Figure 3.3 Enforced expression of RSK kinase domain mutants does not affect erythroid differentiation of primary progenitors

Figure 3.4 Transient knockdown of RSKs 1 and 2 does not impair erythroid differentiation .103

Figure 3.5 Aconitase inhibition prevents ERK-directed phosphorylation of MNK .....106 Figure 3.6 MEK activation is not dynamic in EPO-stimulated erythroid progenitors...109

Figure 3.7 Elk-1 activation is not dynamic in EPO-stimulated erythroid progenitors...112

Figure 3.8 Increased MKP-3 total protein in FA-treated erythroid progenitors .115

Figure 3.9 Aconitase blockade does not affect methylation of PP2A catalytic subunit 118

Figure 3.10 Pharmacological modulation of PP2A catalytic domain activity does not affect erythroid proliferation, differentiation, or viability

Figure 3.11 Increased levels of ERK1/2 in the insoluble compartment of fractionated FA-treated erythroid progenitors

Figure 3.12 ERK2 dimers are constitutively assembled in $\mathrm{CD}_{3} 4^{+}$erythroid progenitors in the absence of EPO.

Figure 3.13 The red cell compartment of ERK1 -/- mice is partially resistant to the effects of FA

Figure 3.14 Effect of aconitase inhibition on the bone marrow erythroid compartment of WT and ERK1 -/- mice.

Figure 3.15 Exogenous isocitrate does not reverse FA-mediated differentiation and signaling defect in primary erythroid cultures 138

Figure 3.16 Isocitrate does not correct FA-induced anemia in wild type mice 140 


\section{LIST OF TABLES}

\section{CHAPTER 1}

Table 1.1 Signaling pathways activated by EPO-EPOR interactions in erythroid progenitors

Table 1.2 Characteristic CBC findings in anemias of varying etiologies

\section{CHAPTER 2}

Table 2.1 Hematologic parameters of C57BL/6 mice treated for 14 days with saline $(0.9 \%)$ or FA $(4 \mathrm{mg} / \mathrm{kg} / \mathrm{day})$

Table 2.2 CBC parameters of WT mice treated for four weeks with saline $(0.9 \%)$ or FA $(2 \mathrm{mg} / \mathrm{kg} / \mathrm{day})$

Table 2.3 CBC parameters of PV mice treated for four weeks with saline $(0.9 \%)$ or FA $(2 \mathrm{mg} / \mathrm{kg} / \mathrm{day})$

\section{CHAPTER 3}

Table 3.1 Baseline platelet and white blood cell counts of WT and ERK1 -/- mice .....132 Table 3.2 Platelet and white blood cell counts of WT and ERK1 -/- mice treated for four weeks with $2 \mathrm{mg} / \mathrm{kg} /$ day FA 


\section{LIST OF ABBREVIATIONS}

$\begin{array}{ll}\text { AI } & \text { Anemia of Inflammation } \\ \text { AMPK } & \text { AMP Kinase } \\ \text { ANC } & \text { Absolute Neutrophil Count } \\ \text { ANOVA } & \text { Analysis Of Variance } \\ \text { APF } & \text { 3'-(aminophenyl)-fluorescein } \\ \text { ATP } & \text { Adenosine Triphosphate } \\ \text { BFU-E } & \text { Burst Forming Unit-Erythroid } \\ \text { BSA } & \text { Bovine Serum Albumin } \\ \text { CBC } & \text { Complete Blood Count } \\ \text { CD } & \text { Cluster of Differentiation } \\ \text { CFU-E } & \text { Colony Forming Unit-Erythroid } \\ \text { CLP } & \text { Common Lymphoid Progenitor } \\ \text { CLS } & \text { Coffin Lowry Syndrome } \\ \text { CMP } & \text { Common Myeloid Progenitor } \\ \text { DDF } & \text { 1,9-Dideoxy Forskolin } \\ \text { DUSP } & \text { Dual Specificity Phosphatase } \\ \text { ERK } & \text { Extracellular-Regulated Kinase } \\ \text { EGF } & \text { Epidermal Growth Factor } \\ \text { EPO } & \text { Erythropoietin } \\ \text { EPOR } & \text { Erythropoietin Receptor } \\ \text { ESA } & \text { Erythropoiesis Stimulating Agent } \\ \text { ET } & \text { Essential Thrombocythemia } \\ \text { FA } & \text { Fluoroacetate } \\ \text { FASL } & \text { FAS Ligand } \\ \text { FACS } & \text { Fluorescence Assisted Cell Sorting } \\ \text { FC } & \text { Fluorocitrate } \\ \text { FSC } & \text { Forward Scatter } \\ & \end{array}$




\begin{tabular}{|c|c|}
\hline GMP & Granulocyte Macrophage Progenitor \\
\hline GPA & Glycophorin A \\
\hline $\mathrm{Hb}$ & Hemoglobin \\
\hline HCT & Hematocrit \\
\hline HEK & Human Embryonic Kidney \\
\hline $\mathrm{HG}$ & Hydroxyglutarate \\
\hline HIF & Hypoxia Inducible Factor \\
\hline HSC & Hematopoietic Stem Cell \\
\hline IC & Isocitrate \\
\hline $\mathrm{IC}_{50}$ & Inhibitory Concentration 50 \\
\hline IDA & Iron Deficiency Anemia \\
\hline $\mathrm{IDH}$ & Isocitrate Dehydrogenase \\
\hline IL & Interleukin \\
\hline IMDM & Iscove's Modified Dulbecco Medium \\
\hline IP3 & Inositol $1,4,5$ triphosphate \\
\hline IRE & Iron Response Element \\
\hline IRP & Iron Response Protein \\
\hline ISC & Iron Sulfur Cluster \\
\hline JAK & Janus Kinase \\
\hline $\mathrm{LDH}$ & Lactate Dehydrogenase \\
\hline LMPP & Lymphoid Primed Multipotent Progenitor \\
\hline MAPK & Mitogen Activated Protein Kinase \\
\hline $\mathrm{MCV}$ & Mean Corpuscular Volume \\
\hline $\mathrm{MCHC}$ & Mean Corpuscular Hemoglobin Concentration \\
\hline MDS & Myelodysplastic Syndrome \\
\hline MEK & MAPK/ERK Kinase \\
\hline MEP & Megakaryocyte-Erythroid Progenitor \\
\hline MFI & Mean Fluorescence Intensity \\
\hline MKP & MAP Kinase Phosphatase \\
\hline MMP & Mitochondrial Membrane Potential \\
\hline
\end{tabular}




\begin{tabular}{|c|c|}
\hline MNK & MAPK-Interacting Kinase \\
\hline MPP & Multipotent Progenitor \\
\hline MSK & Mitogen- and Stress-activated Kinase \\
\hline n.s. & Not significant \\
\hline $\mathrm{OA}$ & Okadaic Acid \\
\hline PBS & Phosphate Buffered Saline \\
\hline PHZ & Phenylhydrazine \\
\hline $\mathrm{PI} 3 \mathrm{~K}$ & Phosphatidylinositol 3-Kinase \\
\hline $\mathrm{PKC}$ & Protein Kinase $\mathrm{C}$ \\
\hline PLC & Phospholipase C \\
\hline PLK & Polo-Like Kinase \\
\hline $\mathrm{PMF}$ & Primary Myelofibrosis \\
\hline $\mathrm{PP} 2 \mathrm{~A}$ & Protein Phosphatase 2A \\
\hline PSP & Protein Serine Phosphatase \\
\hline PTP & Protein Tyrosine Phosphatase \\
\hline PV & Polycythemia Vera \\
\hline $\mathrm{RBC}$ & Red Blood Cell \\
\hline RPMI & Roswell Park Memorial Institute \\
\hline RSK & Ribosomal S6 Kinase \\
\hline ROS & Reactive Oxygen Species \\
\hline $\mathrm{SCF}$ & Stem Cell Factor \\
\hline SiRNA & small interfering RNA \\
\hline SD & Standard Deviation \\
\hline SDS-PAGE & Sodium Dodecyl Sulfate Poly Acrilamide Gel Electrophoresis \\
\hline SEM & Standard Error of the Mean \\
\hline $\mathrm{SSC}$ & Side Scatter \\
\hline STAT & Signal Transducer and Activator of Transcription \\
\hline TfR & Transferrin Receptor \\
\hline TRPC & Transient Receptor Potential Cation Channel \\
\hline pVHL & von Hippel Lindau \\
\hline
\end{tabular}


UA Unexplained Anemia

UTR Untranslated Region

WT Wild Type 


\section{ACKNOWLEDGMENTS AND DEDICATION}

I have been incredibly fortunate to receive lots of support and encouragements throughout my time as a graduate student. There are many people whom I need to acknowledge who have made my completion of this work possible. First and foremost, I wish to thank my mentor, Adam Goldfarb, a dedicated advisor with a genuine love for teaching. His scientific curiosity and rigor, combined with his continued involvement with clinical work, have shown me how to be a successful physician scientist. I also must acknowledge all members of the Goldfarb lab: Lorrie Delehanty, Chanté Richardson, Grant Bullock, Kamal Elagib, Jeremy Rubinstein, and Ivo Mihaylov (former member). I am indebted to Lorrie and Grant in particular; this work would not have come together without their guidance and technical expertise. Many thanks also to Dr. Janet Cross and Michael Kidd in the Department Pathology for their continued support. I would like to thank fellow graduate students in the Department of Pathology and my classmates in the UVA Medical Scientist Training Program for wonderful friendships, my family for their encouragements throughout my life, and last but not least my partner and greatest supporter, David Talbot. This work is dedicated to my parents, Claude and Claudine Allary. 
CHAPTER 1:

General Introduction 


\subsection{Overview of hematopoiesis}

Hematopoiesis is the physiological process by which a small population of selfrenewing pluripotent hematopoietic stem cells (HSCs) give rise to all differentiated blood cell types [1]. Upon transplant, HSCs can reconstitute the entire hematopoietic system of a lethally irradiated host, a seminal discovery made fifty years ago by Till and McCulloch [2]. A single HSC can undergo symmetric division, yielding two identical daughter HSCs, or asymmetric division, where one daughter retains the pluripotent characteristics of its parent (self-renewal) and the other commits to a specific differentiation program [3]. The factors regulating HSC fate are still under investigation; the current model emphasizes key roles for intrinsic (i.e., transcription factors) and extrinsic factors (i.e., growth factors) [4].

According to the prevalent model, HSCs give rise to two multipotent progenitors; the common lymphoid progenitor (CLP) cell and the common myeloid progenitor (CMP) cell (Figure 1.1). The CLP eventually gives rise to T and B lymphocytes and NK cells, while the CMP is a common progenitor for red cells, platelets, neutrophils, monocytes, basophils, and eosinophils [5,6]. As progenitor cells become lineage-restricted, they progressively lose their self-renewing potential; mature blood cells have no regenerative capabilities. Stage-specific upregulation and downregulation of a cohort of transcription factors is critical to ensure hematopoietic differentiation. The recent discovery of a subset of HSCs with myeloid and lymphoid potential but unable to yield megakaryocyte 
Figure 1.1 Current and alternative models for hematopoietic stem cell and blood lineage commitment. (A) Current model of blood lineage commitments based on studies of Weissman and colleagues. (B) Alternative model proposed by Adolfsson et al. based on the identification of a multipotent progenitor that cannot yield erythroid and megakaryocytic colonies. (C) Composite model devised by Adolfsson et al., integrating the findings of the Weissman group with their own. LT-HSC, long-term hematopoietic stem cell; ST-HSC, short-term hematopoietic stem cell; MPP, multipotent progenitor; LMPP, lymphoid-primed multipotent progenitor; CLP, common lymphoid progenitor; CMP, common myeloid progenitor; GMP, granulocyte/macrophage progenitor; MkEP, megakaryocyte/erythroid progenitor; B, B cell; T, T cell. (Adapted from Adolfsson et al., 2005) 
Figure 1.1 Current and alternative models for hematopoietic stem cell and blood lineage commitment.

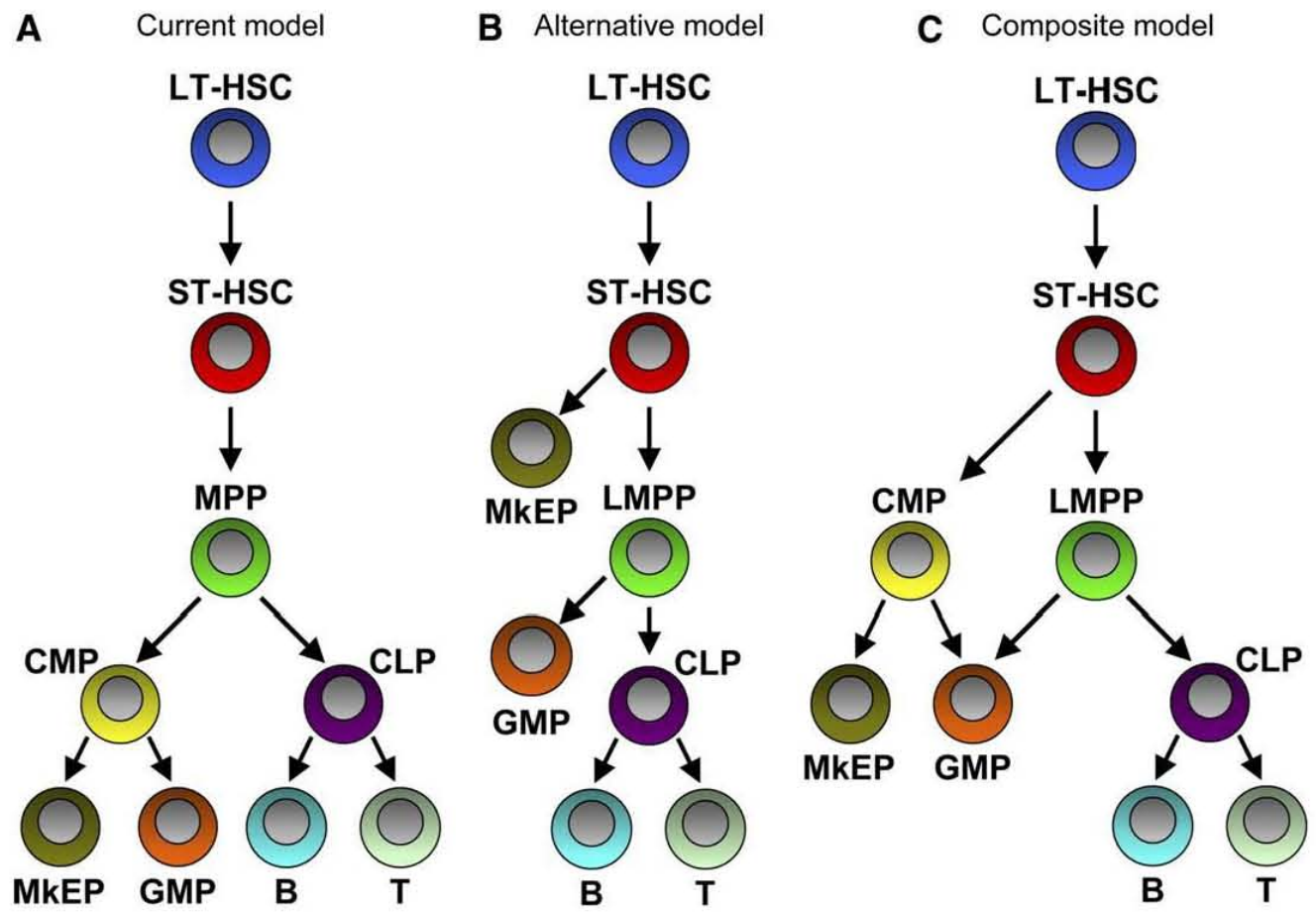


or erythroid colonies suggests that the roadmap of blood lineages may need to be redrawn [7]. This alternate model argues for earlier divergence between the MEP and a common Lymphoid-Primed Multipotent Progenitor capable of yielding lymphoid and myeloid cells (Figure 1.1B). While fascinating, a discussion of HSC biology and hematopoietic lineages other than the erythroid lineage is beyond the scope of this dissertation.

\subsection{Erythroid development}

\subsubsection{Commitment to the erythroid lineage}

Under the influence of extrinsic and intrinsic factors, the CMP cell can give rise to two progenitor cells, Megakaryocyte-Erythroid Progenitors (MEPs or MkEPs) and Granulocyte and Macrophage Progenitors (GMP) (Figure 1.1A). MEP cells are the last bipotent progenitors before lineage commitment. MEPs give rise to either megakaryocyte or erythroid progenitors. Several genetic factors that control fate decision in MEP cells have been identified; these include the transcriptions factors GATA1, FOG1, RUNX1, Fli-1, c-Myb, EKLF, and more recently, STAT5 [8-13]. Some of these factors are lineage-specific; this is the case of RUNX1 and Fli-1, two critical factors for megakaryocytic differentiation, and EKLF, an erythroid-specific transcription factor essential for $\beta$-globin synthesis [14]. Several factors, however, are shared between the megakaryocytic and erythroid lineages, and specificity is provided by gene dosage rather than by the presence or absence of a given transcription factor [14]. For instance, 
GATA1 exerts a regulatory role during the ontogeny of megakaryocytes, erythrocytes, mast cells, and eosinophils; four cell types with vastly different functions [15-17]. One way to achieve specificity is through lineage-specific GATA1 regulatory sequences in promoters of target genes. This allows fine-tuning of the concentration of GATA1 within a given cell. In addition, regulatory sequences in the GATA1 gene itself ensures appropriate GATA1 levels in a given cell [18].

\subsubsection{Erythroid developmental stages}

Definitive erythropoiesis takes places in the bone marrow, in contrast to embryonic and fetal erythropoiesis, which take place in the yolk sac and liver, respectively [19]. Upon commitment to the erythroid lineage, cells are classified functionally into progenitor (early) and precursor (late) populations. As detailed in Figure 1.2, erythroid progenitor cells are composed of morphologically indistinguishable Burst Forming Colony-erythroid (BFU-e) cells and Colony Forming Unit-erythroid (CFU-e) cells that represent a continuum of differentiation. BFU-e cells are the earliest committed erythroid progenitors and are identified by their ability to form large colonies of hemoglobinized cells when plated in semi-solid medium [20]. BFU-e cells have some self-renewing capabilities, as evidenced by the appearance of colonies upon replating. Most BFU-e cells are dormant, but are highly proliferative in vitro upon entering the cell cycle. The average time to erythroid colony formation upon plating is 14 days. The next stage is the Colony Forming Unit-erythroid. CFU-e cells are for the most part cycling at 
any given time, have less proliferative capacity than BFU-e cells, and cannot self-renew. The average time from CFU-e plating to erythroid colony formation is 7 days. In addition to replating characteristics, molecules expressed on the surface of progenitors can be used to distinguish BFU-e from CFU-e cells. Cluster of differentiation 34 (CD34) is a cell surface glycoprotein expressed in BFU-e cells, but not in CFU-e cells [21]. It is also expressed in all other hematopoietic progenitors and in vascular endothelial cells.

Erythroid precursors can be staged developmentally using a combination of morphological features and cell surface markers. The first morphologically recognizable erythroid precursor and direct progeny of the CFU-e is the proerythroblast. Precursor cells go through three additional stages - basophilic, polychromatophilic, and orthochromatic stages - before undergoing enucleation and becoming reticulocytes (Figure 1.2). In addition to nuclear extrusion, terminal maturation requires clearance of cytoplasmic organelles, including mitochondria, Golgi remnants, and ribosomes, and extensive membrane remodeling [22-24]. Mature erythrocytes enter the bloodstream carrying little else but hemoglobin and have highly deformable membranes, which make them optimally suited to transport oxygen to all tissues. Cell surface markers commonly used to stage erythroid precursors include Glycophorin A (GPA) and Transferrin Receptor (TfR). Glycophorin A, also referred to as CD235a, is sialoglycoprotein expressed on the surface of erythroid precursors, beginning at the CFU-e stage (Figure 1.2) [25]. Its expression increases continually throughout terminal differentiation. TfR molecules, or CD71, are abundantly expressed in erythroid precursors, and their 
Figure 1.2 The erythroid differentiation pathway. The differentiation pathway from the first committed erythroid progenitor cell (BFU-e) to the mature circulating red cell is depicted based on the morphological, functional and antigenic characteristics of erythroid progenitor cells during differentiation. The association of membrane expression of a particular receptor or antigen is not meant to imply functionality but only the potential for expression at the particular stage of erythroid differentiation. CD34, an adhesive protein that is expressed by primitive hematopoietic progenitor cells; CD36, an adhesive protein expressed by erythroid progenitor cells; CD41, a membrane protein expressed by MEPs, megakaryocytes and endothelial cells; CD71, the transferrin receptor; CXCR4, the stromal-cell-derived factor-1 $\alpha$ receptor; EPOR, erythropoietin receptor; KIT, Stem Cell Factor receptor; IL-3R, interleukin 3 receptor. (Adapted from Spivak, 2005) 
Figure 1.2 The erythroid differentiation pathway.

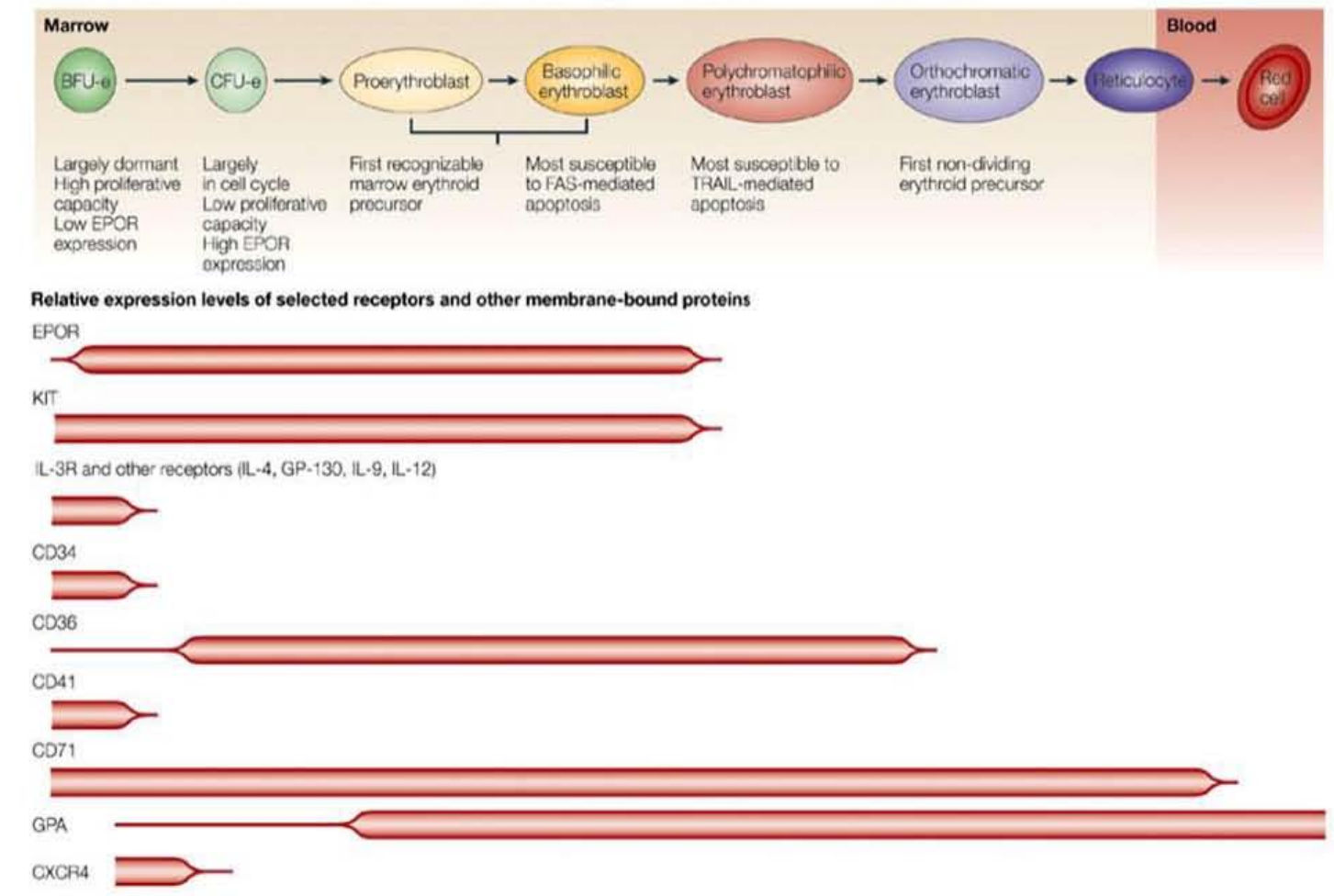


expression is downregulated as cells transition from reticulocytes to mature red blood cells [25]. The combined pattern of GPA and CD71 expression has been extensively used to break down erythroid precursor populations into distinct stages of maturation by flow cytometric analysis [26].

\subsubsection{The erythroblastic island}

Erythroid development from the proerythroblast stage through the orthochromatic stage takes places in specialized structures within the bone marrow called erythroblastic niches or islands. These structures consist of a centrally-located macrophage cell surrounded by a ring of erythroblasts [27]. Adhesions between the macrophage and erythroid progenitors appear to be key for the development of the latter. These points of contact are created by cytoplasmic protrusions emanating from the macrophage, as identified by electron microscopy [28]. Of the several molecules shown to mediate macrophage-erythroblast interaction, expression of $\alpha 4 \beta 1$ integrin on the erythroblast cell surface and VCAM-1 on the macrophage cell surface is essential [29]. The macrophages within erythroblastic islands have been shown to serve functions essential for erythroid maturation: secretion of cytokines such as IGF-1 with positive effects on growth of CFUe progenitors and phagocytosis of extruded nuclei [30]. They may also facilitate iron transit and acquisition by the erythroid precursors. Lastly, direct contact between neighboring erythroblasts are thought act as a sensor of precursor mass and can regulate final erythroid output through FAS-FAS ligand-mediated signaling (see section 1.3.5). 


\subsection{Regulation of erythropoiesis}

\subsubsection{EPO-EPOR signaling}

Erythropoietin is a 166 amino acid, $35 \mathrm{kDa}$ glycopeptide synthesized by the peritubular cells of the kidney that is absolutely required for definitive erythropoiesis [31]. Its existence was postulated as early as the 1950s and was first isolated from urine of patients with aplastic anemia. The EPO gene was cloned in 1985 [32]. The cell surface receptor for EPO, EPOR, was cloned in 1989 and was shown to be essential for the proliferative and survival functions of EPO, despite lacking a functional cytoplasmic kinase domain $[33,34]$. EPOR is a 507 amino acid protein with a single transmembrane domain that belongs to the cytokine receptor superfamily. Binding of EPO induces dimerization of the receptor and interaction with cytoplasmic kinases. Shortly after the EPOR was cloned, the cytoplasmic kinase JAK2 was identified and shown to be required to mediate the mitogenic effects of EPO [35]. The EPO-induced conformational change in EPOR triggers activation of JAK2 molecules pre-bound to its cytoplasmic tail. Autophosphorylation of JAK2 and subsequent cross phosphorylation of tyrosine residues on the EPOR cytoplasmic tail creates recruitment sites for adapter proteins with Src homology 2 (SH2) domains and further binding of signal transducing proteins, including p85 and STAT5 [36,37].

Functional requirement for EPO, EPOR, and JAK2 during erythropoiesis has been demonstrated in mouse knockout models. Germline deletions of any of these genes are 
embryonic lethal [38-40]. EPOR is expressed on BFU-e progenitors and upregulated as the cells transition to the CFU-e stage. Autoradiography-based studies have established that cell surface EPOR expression is at its highest at the transition from CFU-e to proerythroblast [41]. BFU-e and CFU-e colonies can be derived in the absence of EPO or EPOR, but these colonies cannot produce terminally differentiated erythrocytes.

\subsubsection{STAT5 signaling}

The actions of EPO are mediated by multiple intracellular signaling pathways. A list of experimentally described EPO-stimulated pathways is presented in Table 1.1. Historically, JAK2-mediated activation of STAT5 has been touted as the critical pathway in erythroid development. Activation of JAK2 upon EPO-EPOR interaction allows recruitment and activation of Signal Transducer and Activator of Transcription 5 (STAT5). Specifically, it was shown that a single tyrosine residue, Y343, in the EPOR cytoplasmic tail was necessary for STAT5 activation to occur [42]. STAT5 was identified in the mid 1990s and found to be activated by the hormone prolactin [43]. Cloning of the murine homolog revealed that STAT5 consists of two closely related proteins: STAT5a and STAT5b. Initial characterization of STAT5a -/- b -/- mice failed to show any defect in the red cell compartment of these animals [44]. EPO-induced ex vivo colony formation of bone marrow-derived cells was also unimpaired. However, studies of fetal erythropoiesis in these animals revealed profound anemia in 
Table 1.1. Signaling pathways activated by EPO-EPOR interactions in erythroid progenitors

\begin{tabular}{|c|c|c|}
\hline Pathway & Effect on erythropoiesis & References \\
\hline JAK2 - STAT5 & Survival & $\begin{array}{l}{[45]} \\
{[26]}\end{array}$ \\
\hline ERK1/2 & Proliferation & [46] \\
\hline p38 & Terminal differentiation & $\begin{array}{l}{[47]} \\
{[48]}\end{array}$ \\
\hline$\overline{\text { JNK }}$ & Proliferation & $\begin{array}{l}{[47]} \\
{[49]}\end{array}$ \\
\hline PI3K - Akt & Survival & $\begin{array}{l}{[50]} \\
{[51]} \\
{[52]}\end{array}$ \\
\hline PKC $\alpha / \beta I I$ & $\begin{array}{l}\text { Differentiation } \\
\text { Regulation of EPOR signaling }\end{array}$ & $\begin{array}{l}{[53]} \\
{[54]}\end{array}$ \\
\hline PLC $\gamma$ & $\begin{array}{l}\text { Differentiation } \\
\text { EPO-mediated calcium signaling }\end{array}$ & $\begin{array}{l}{[55]} \\
{[56]}\end{array}$ \\
\hline
\end{tabular}


STAT5a -/- b -/- embryos due to increased apoptosis [45]. The studies of Socolovsky et al. demonstrated direct regulation of $\mathrm{Bcl}-\mathrm{X}_{\mathrm{L}}$, a transcription factor well-known to promote survival in erythroid progenitors by preventing apoptosis, by the EPO-JAK2STAT5 axis [57]. Follow-up studies showed that STAT5a/b is specifically required for the survival of early erythroblasts, and that adult mice lacking STAT5a/b with normal red cell parameters at baseline fail to respond to acute anemic stress [26]. The precise contribution of STAT5a/b signaling during EPO-mediated erythropoiesis was challenged when mice bearing a truncated form of EPOR were studied. Zang et al. generated mice lacking all cytoplasmic tyrosine residues in EPOR but Y343 (EPO H mice) and Y343F mutant mice also lacking all other tyrosine residues (EPO HM mice) [58]. The EPO HM mice were predicted to show severe, possibly fatal, anemia due to failure to activate STAT5a/b. However, these mice had a rather mild red cell phenotype, characterized by a modest decrease in hematocrit. EPO HM mice had no splenomegaly, and embryos were indistinguishable from their wild type counterparts, a finding divergent from that reported by Socolovsky et al. [45,58]. Thus significant phenotypic differences between STAT5a /- $\mathrm{b}$-/- and EPO HM mice have raised new questions about the precise role of STAT5a/b in erythropoiesis. One recent study suggests that EPOR Y343 and STAT5a/b activation are critical during the response to acute anemic stress [59]. Surprisingly, BCL- $\mathrm{X}_{\mathrm{L}}$ was not found to mediate this phenotype. Rather additional STAT5a/b target genes as well as surface receptor-activated pathways other than EPO appear to be involved [59]. Thus the pleiotropic effects of STAT5 signaling during erythropoiesis remain to be fully dissected. 


\subsubsection{ERK1/2 signaling}

Another relevant pathway is EPO-mediated activation of Extracellular-Regulated Kinases 1 and 2 (ERK1/2). ERK1/2, also referred to as Mitogen-Activated Protein Kinases (MAPKs), are proline-directed kinases that regulate many cellular processes, including proliferation in response to mitogenic stimuli. The MAPK cascade, a three-tier cascade comprised of the kinases Raf, MEK, and ERK, is activated in response to EPO binding to EPOR. Phosphorylated tyrosine residues in the cytoplasmic tail of EPOR recruit adaptor proteins such as Grb2, which in turn activate Ras and Raf [60]. The importance of ERK signaling in erythroid development was first inferred from studies looking at the consequences of abnormal Ras signaling. Mice lacking K-Ras die in utero with anemia, while overexpression of mutant N-Ras in primary hematopoietic progenitors impairs erythroid differentiation [61,62]. Further studies in fetal livers, a site of abundant erythropoiesis during the fetal period, revealed that oncogenic Ras inhibits differentiation of CFU-e progenitors while promoting EPO-independent growth [63]. These effects were entirely due to constitutive signaling via MEK and ERK [46]. Pharmacologic inhibition of MEK1/2 in erythroleukemic cell lines and primary hematopoietic progenitors favored the formation of erythroid colonies over megakaryocytic colonies and favored erythroid differentiation [64,65]. In addition to proliferation and differentiation, ERK signaling may regulate survival of erythroid progenitors by promoting degradation of the pro-apoptotic protein Bim [66]. 
Figure 1.3 Schematic representation of the ERK signaling cascade. Activation and inactivation processes are indicated. Bold arrows stand for the main pathway upon growth factor activation. Red, activating phosphorylation; green, accessory phosphorylation; blue, inhibitory phosphorylation and dephosphorylation. (Adapted from Shaul and Seger, 2007) 
Figure 1.3 Schematic representation of the ERK signaling cascade.

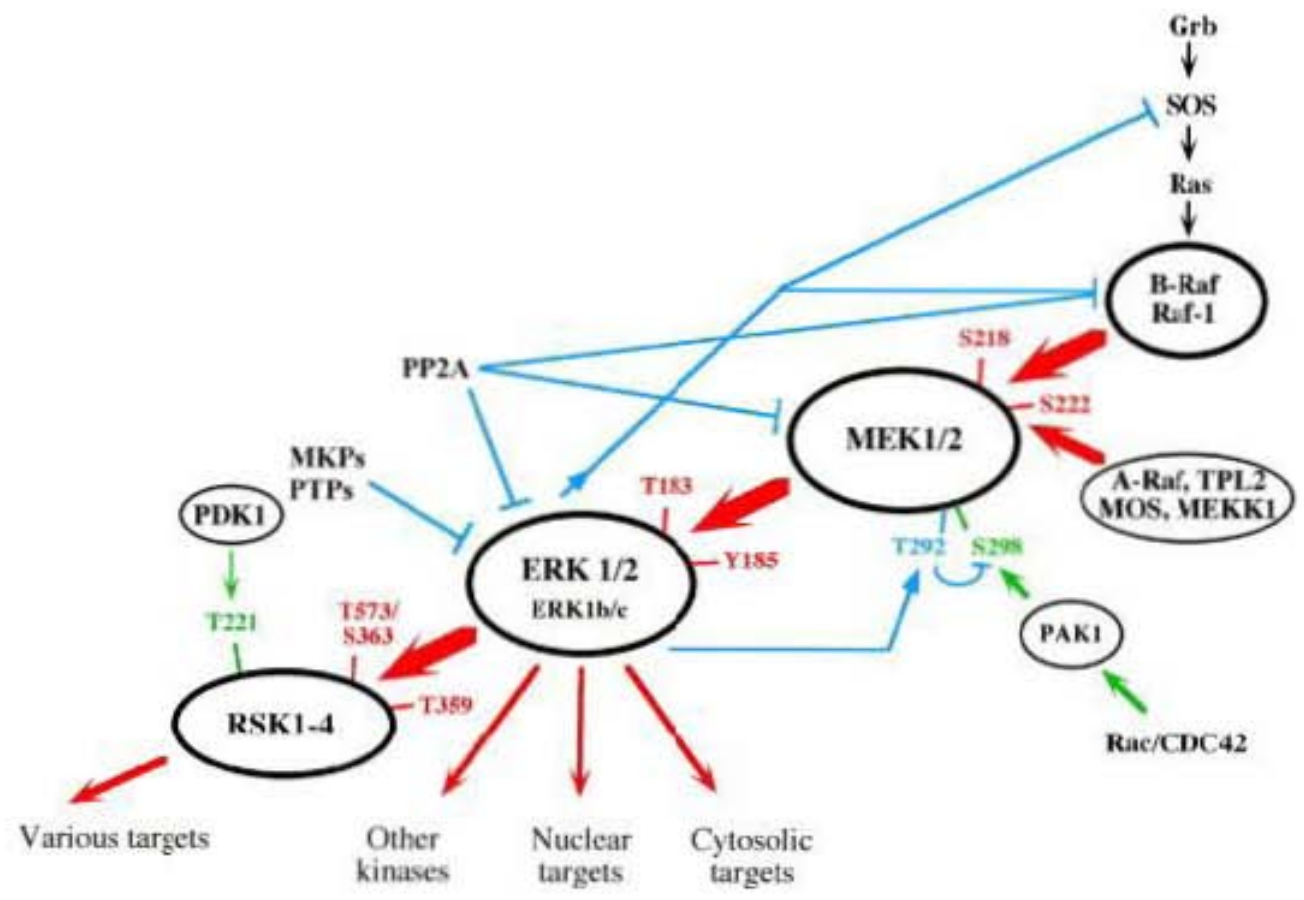


ERK1 and ERK2 are $83 \%$ identical in their protein sequences and are expressed in all tissues at varying levels [67]. They do, however, have non-redundant functions. Analysis of the erythroid compartment in ERK1 -/- mice has suggested that ERK1 may be a negative regulator of erythropoiesis [68]. Mice lacking ERK1 had slightly increased red cell parameters at baseline and responded to acute anemic stress more robustly than wild type mice. ERK2 deletion in mice is embryonic lethal due to severe placental defects; and its specific contribution to erythroid development is not known [69]. ERK1/2 have over 150 know targets located in various subcellular compartments, and the outcome of ERK activation is further regulated by interaction with scaffolds, other signaling cascades, and duration of activation [70].

\subsubsection{Hypoxia}

Decreased oxygen tension has been known to stimulate red cell production since the seminal observations that high altitude correlates with increased levels of circulating red blood cells. Efforts to understand the mechanistic underpinnings of this surge of erythropoietic activity ultimately led to the discovery of the EPO gene and the Hypoxia Inducible Factor (HIF) family of transcription factors. Hypoxic conditions lead to activation of the EPO gene, a response that is orchestrated by HIF-1 [71]. HIF-1 consists of an oxygen-sensitive $\alpha$ subunit and a constitutively expressed $\beta$ subunit. HIF-1 heterodimers bind the EPO gene via a hypoxia response element to activate transcription. Under normoxic conditions, HIF-1 $\alpha$ molecules are hydroxylated on specific proline 
residues by HIF prolyl-hydroxylases, a post-translational modification that is oxygendependent [72][73]. Hydroxylation subsequently targets HIF-1 for degradation via recognition by the von Hippel-Lindau tumor suppressor protein (pVHL). pVHL is part of an E3 ubiquitin ligase complex and targets HIF-1 to the proteasome for degradation [74]. Under hypoxic conditions, failure to hydroxlate HIF-1 leads to its stabilization, formation of active transcription complexes, and upregulation of $E P O$ transcription. In addition to EPO production, the HIF-mediated response to hypoxia enhances iron uptake and utilization and acts directly on the bone marrow to modulate erythroid proliferation and maturation [75-77]. HIF-1 $\alpha$ has been shown to regulate the transcription of key iron metabolism players, including hepcidin and transferrin [78,79].

\subsubsection{Iron}

Bioavailable iron is required by all tissues for multiple processes. Ferrous iron is incorporated in iron-sulfur clusters (ISCs) in the catalytic site of a variety of enzymes participating in cellular metabolism. In developing erythrocytes, there is a large additional demand for iron during heme synthesis. Continuous production of red blood cells at an estimated rate of 200 billion new erythrocytes daily accounts for about $80 \%$ of all iron demands in humans [80]. Upon absorption, dietary iron is transported from the intestinal cells to sites of utilization by transferrin, a carrier protein with a very high

affinity for ferric iron $\left(\mathrm{Fe}^{3+}\right)$. Iron-bound transferrin interacts with transferrin receptors at the cell surface. Transferrin Receptor 1 (TfR1) is ubiquitously expressed. Iron-loaded 
transferrin binds TfR1 and the complex is internalized via endocytosis. Acidification of early endosomes promotes iron release from transferrin, conversion to ferrous iron $\left(\mathrm{Fe}^{2+}\right)$, and release into the cytosol, or possibly direct delivery to the mitochondria, the site of most iron requiring processes $[81,82]$. Transferrin and TfR 1 are subsequently recycled to the cell surface. Transferrin Receptor 2 (TfR2) is expressed only on hepatocytes, duodenal cells, and erythroid cells. TfR2 was recently shown to interact with EPOR complexes and be required for efficient erythropoiesis [83].

Iron levels are critical regulators of erythropoiesis. Genetic deletion of TfR1 causes embryonic death prior to embryonic day E12.5 [84]. TfR1 +/- mice are viable but have hypochromic and microcytic red cells, two hallmarks of iron deficiency anemia. Knockdown of TfR2 delays terminal erythroid maturation [83]. Iron restriction, i.e. diminished amounts of bio-available iron, results in diminished red cell production due to decreased erythroid proliferation and maturation [85]. Iron regulation of erythropoiesis has also been documented in a rat experimental model, where iron deficiency causes a defect in the transition from the CFU-E stage to the proerythroblast stage [86]. We have shown that erythroid progenitors cultured in low iron conditions proliferate and differentiate less, and have a decreased survival capacity when compared to iron-replete control cultures [87]. 


\section{$\underline{1.3 .4 \text { Calcium }}$}

Activation of cytokine receptors such as EPOR does not trigger rapid calcium influx as occurs with antigen receptor activation [88]. Nevertheless, calcium levels can modulate erythropoiesis. Calcium signaling contributes to the differentiation of murine erythroleukemia cells $[89,90]$. Furthermore, EPO was shown to mediate a dosedependent increase in calcium in human BFU-e progenitors via a voltage-independent ion channel [91]. Systematic deletion studies identified key tyrosine residues in the cytoplasmic tail of EPOR necessary for EPO-mediated calcium influx [92].

Subsequently, the Transient Receptor Potential Cation (TRPC) channel family was investigated as possible voltage-independent $\mathrm{Ca}^{2+}$-permeable channels on erythroid progenitors. Studies spearheaded by the Miller lab have shown that TRPC3 is the primary EPO-modulated $\mathrm{Ca}^{2+}$ channel in developing red blood cells [56][93]. EPO promotes $\mathrm{Ca}^{2+}$ influx in a dose-dependent manner, likely via TRPC3-Phospholipase C- $\gamma$ (PLC $\gamma$ ) interactions. PLC $\gamma$ catalyzes the hydrolysis of phosphatidylinositol 4,5bisphosphate to inositol 1,4,5, triphosphate (IP3) and diacylglycerol. IP3 is known to trigger $\mathrm{Ca}^{2+}$ release from the endoplasmic reticulum [94].

\section{$\underline{1.3 .5}$ FAS-FAS ligand}

FAS ligand (FASL), or CD95L, is a member of the Tumor Necrosis Alpha (TNF) family of death ligands. Its cognate receptor, FAS, or CD95, belongs to the TNF death 
receptor family [95]. FASL and FAS are expressed on the surface of erythroid progenitors, starting at the BFU-e stage [96]. Cross-linking of ligand and receptor leads to caspase-mediated apoptosis [97]. Interestingly, the ability of FAS-FASL to trigger apoptotic cell death requires that the cells be cycling; quiescent $\mathrm{CD} 34^{+}$cells were shown to be resistant to FAS-mediated apoptosis. Furthermore, detailed studies revealed that immature erythroid precursors express more FAS, while more mature precursors have high levels of FASL. Thus mature precursors can negatively regulate the expansion of early precursors by FAS-FASL cross-linking. This interaction is inhibited when EPO levels are high, suggesting that FAS-FASL functions as a negative feedback loop to blunt ineffective proliferation when EPO levels are suboptimal [98]. In a model of stress erythropoiesis, EPO-mediated downregulation of FAS-FASL expression on splenic erythroblasts allowed for the rapid expansion of progenitors [99]. In a separate study, caspase-dependent cleavage of GATA1 secondary to FAS-FASL interactions was proposed as the mechanism mediating arrest of erythroid differentiation under low EPO conditions [100].

\subsection{Aconitases}

\subsubsection{Enzymatic function}

The aconitase proteins are members of the hydratase family of enzymes (EC 4.2.1.3) [101]. They reversibly interconvert the metabolites citrate and isocitrate in a 
two-step reaction that yields a short-lived intermediate, cis-aconitate [101]. This catalytic reaction depends on the presence of an intact [4Fe-4S] ISC in the enzyme's active site [101]. Shortly after their discovery in 1937, it was found that cells have a mitochondrial aconitase isoform (m-aconitase) and a cytosolic one (c-aconitase). Each isoform is encoded by separate genes located on different chromosomes. M-aconitase is encoded by a nuclear gene located on chromosome 22 [102]. The gene coding for c-aconitase is located on chromosome 9 [103]. In the mitochondria, aconitase participates in the citric acid cycle and contributes to the production of ATP and NADPH. Cytosolic aconitase is a free-standing enzyme. Citrate is an important intermediate in several cellular energyproducing metabolic pathways. Cytoplasmic citrate can be converted to acetyl-coenzyme A by ATP-citrate lyase, which is a critical building block for cholesterol and fatty acid synthesis $[104,105]$.

\subsubsection{Regulation of catalytic activity}

Aconitase activity can be regulated by several mechanisms. A first layer of regulation occurs at level of the ISC. Defects in the ISC biogenesis machinery, such as deficiency in the mitochondrial protein frataxin, precludes assembly of functional clusters [106]. Frataxin has been shown to interact with scaffold proteins involved in ISC assembly and may function as a chaperone for iron incorporation in the ISC. Clinically, frataxin deficiency causes Friedreich's ataxia, a neurodegenerative disease characterized by decreased heme synthesis, mitochondrial iron overload, and oxidative damage 
$[107,108]$. The alpha iron of the ISC is extremely labile and [3Fe-4S] clusters are unstable. Reactive oxygen species, including hydrogen peroxide and peroxynitrite, can rapidly inactive aconitase [109-111]. The enzymatic activity of both aconitase isoforms can also be modulated by phosphorylation. In diabetic rat hearts, mitochondrial aconitase was found to be phosphorylated after PKC activation [112]. Interestingly, this phosphorylation event correlated with increased reverse catalytic activity, while the rate of citrate to isocitrate conversion remained unchanged. Conversely, phosphorylation of serine residue 711 of cytosolic aconitase by PKC impairs the forward reaction, while the conversion of isocitrate back to citrate is unimpaired [113].

Aconitase activity can be inhibited pharmacologically by the small molecule fluoroacetate (FA), a precursor of fluorocitrate (FC). Over fifty years ago, FC was shown to be produced by citrate synthase and to inhibit the catalytic activity of aconitase [114,115]. Reaction of FC with aconitase and subsequent defluorination yields hydroxyaconitate, a reactive intermediate that cannot be displaced from the active site despite addition of up to 20 molar excess citrate, leading to stable inhibition [116]. These data were later confirmed by solving the crystal structure of FC-aconitase complexes [117]. It has also been suggested that FC can inhibit transport of certain carboxylic acids [118]. Excessive FA intake primarily affects the cardiovascular and central nervous systems, and can cause death quickly $[119,120]$. At lower doses however, FA has been a useful tool to dissect the relative contribution of mitochondrial aconitase activity to overall ATP production and citrate level regulation in astrocytes and prostate cell lines [121,122]. 


\section{$\underline{\text { 1.4.3 Cytosolic aconitase }}$}

In addition to its catalytic function, c-aconitase has RNA-binding properties and can function as an iron regulatory protein, hence its alternate name, Iron Regulatory Protein 1 (IRP1) [123]. These two functions are mutually exclusive; the protein exists either as an aconitase or as an IRP [124]. When iron levels dip below a certain threshold, IRP1 binds Iron Responsive Element (IRE) sequences in the 3' or 5' untranslated region (UTR) of messenger RNA (mRNA) of many genes involved in iron metabolism. IREs are short hairpin structures composed of 30 nucleotides on average. There may be several IREs in the UTR of a given iron metabolism gene. The opposing regulation of $T f R 1$ and ferritin, an intracellular iron storage protein, is the classical example and is depicted in Figure 1.4 [125-127]. Under low iron conditions, TfR1 mRNA is stabilized while translation of ferritin mRNA is reduced, leading to increase iron uptake and decrease storage in order to maximize intracellular iron availability. Under high iron conditions, IRE binding is decreased, leading to less TfR1 and increased ferritin protein synthesis for enhanced iron storage.

IRP2 is another cytoplasmic post-transcriptional regulator of IRE-containing

mRNAs. IRP1 and IRP2 are close homologues, sharing 57\% sequence homology [123]. IRP2 -/- mice show abnormal regulation of iron homeostasis in their intestinal mucosa and central nervous system, as well as cerebellar neurodegeneration, and develop a microcytic anemia $[128,129]$. These results indicated that IRP1 cannot compensate for loss of IRP2. Generation of IRP1 -/- mice further helped tease out important functional 
Figure 1.4 Iron-dependent regulation of IRP binding activity. Under conditions of iron deficiency, IRP1 and IRP2 bind to IREs, and thus repress mRNA translation (A) or prevent mRNA degradation (B). Increased iron levels lead to the loss of IRP affinity for IREs, and increase the translation of 5' IRE-containing mRNAs and the degradation of 3' IRE-containing mRNAs. (Adapted from Recalcati, 2010) 
Figure 1.4 Iron-dependent regulation of IRP binding activity.
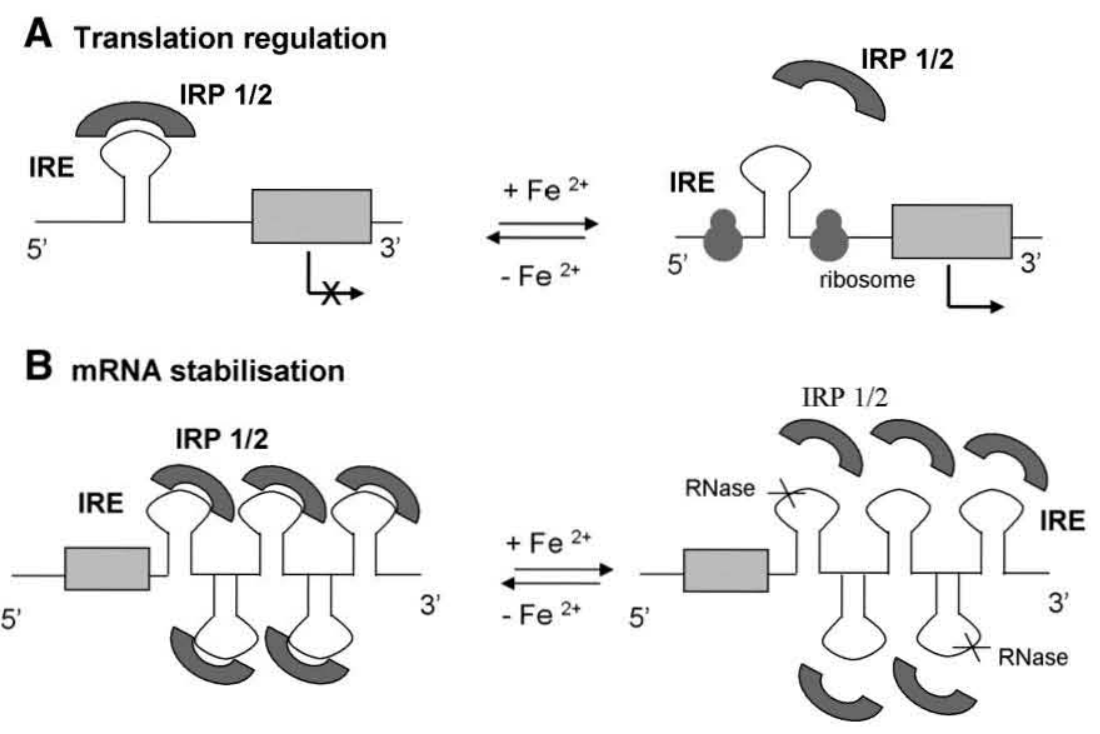
differences between the two IRP proteins. As opposed to their IRP2 -/- counterparts, mice lacking IRP1 have no overt phenotype [130]. Studies have shown that IRP1 exists predominantly in its c-aconitase form and is not recruited to regulate iron metabolism, even when mice are kept on a low iron diet, and that IRP2 levels increase in IRP1 -/animals. Thus IRP2 appears to be the primary regulator of iron responsive genes [130].

\subsubsection{Mitochondrial aconitase}

Mitochondrial aconitase is an essential member of the citric acid cycle, converting citrate to isocitrate. This step is reversible and not rate-limiting. Isocitrate is further metabolized to $\alpha$-ketoglutarate by isocitrate dehydrogenase, a reaction that yields one NADH molecule. The 5' UTR of the m-aconitase mRNA contains an IRE, and the relative abundance of $\mathrm{m}$-aconitase can thus be regulated by iron levels via IRP proteins. [131]. As predicted, iron loading increased m-aconitase protein levels while iron depletion diminishes its abundance [132]. It has been suggested that iron regulation of m-aconitase levels may couple citrate metabolism to iron content, possibly because citrate is a known iron chelator $[132,133]$. More recently, m-aconitase was found in mitochondrial nucleoids, a cluster of proteins that assist in packaging of mitochondrial DNA (mt DNA) and shown to be essential for mtDNA maintenance [134]. M-aconitase was subsequently shown to have intrinsic double-stranded mtDNA binding activity [135]. Direct binding of m-aconitase to mtDNA appears to be essential to protect the mitochondrial genome from point mutation and other forms of DNA damage [135]. 


\subsubsection{Aconitases in erythropoiesis}

Both the cytosolic and mitochondrial isoforms of aconitase are intimately linked to erythropoiesis. C-aconitase, in its IRP1 form, can regulate translation of iron metabolism genes, while m-aconitase contributes to heme synthesis by generating isocitrate, a precursor for the heme building block succinyl CoA $[123,136]$. Recent studies in our laboratory identified the aconitases as mediators of the response to iron deprivation in vitro and in vivo [87]. Primary erythroid progenitor cells maintained in low iron conditions exhibited decreased proliferation and differentiation and diminished aconitase activity in a lineage-restricted manner. Only progenitors cultured in erythroid differentiation medium and low iron underwent aconitase inactivation. Importantly, provision of isocitrate rescued erythroid differentiation in these cultures. Isocitrate was also shown to transiently reverse anemia in mice fed an iron-deficient diet [87].

\subsection{Clinical connections}

\section{$\underline{1.5 .1 \text { Iron deficiency anemia }}$}

Iron deficiency anemia (IDA) has been a long-standing public health issue worldwide, with an estimated affected population of nearly 3 billion [137]. The anemic condition has been recognized for centuries, and formally proven to be due to iron deficiency in the 1930s when parenteral administration of iron was shown to correlate 
with an increase in hemoglobin [138]. Iron deficient status most often results from blood loss, which can arise from trauma or gastrointestinal bleeding secondary to a parasitic infection such as Helicobacter pylori (H. pylori), as well as inadequate dietary intake. Menstruating women are at an increased risk of IDA due to low iron reserves at baseline. Rare mutations in genes involved in iron metabolism and homeostasis can also be the cause of IDA. This is the case with a point mutation in the DMT1 gene, which encodes an iron transporter at the apical surface of duodenal enteroctytes, and transferrin mutations, which prevent transport and delivery of gut-absorbed iron to erythroid progenitors [139-141]. As detailed in section 1.3.3, iron deficiency impairs erythroid proliferation and differentiation [85]. The classic findings in the complete blood count of a patient with IDA include microcytic, hypochromic red cells that are diminished in number. This is in contrast to anemias not due to iron deficiency, as outlined in Table 1.2. First-line treatment of IDA consists in replenishing iron stores via oral iron therapy. Intravenous iron can be used as second line therapy in patients with intestinal malabsorption or on concurrent EPO therapy secondary to chronic kidney disease. In the case of IDA caused by H. pylori infection, eradication of the bacterium can lead to complete reversal of the anemia [142]. Red cell transfusion is not recommended for the treatment of IDA. 


\subsubsection{Polycythemia Vera}

Disorders of red cell production can also manifest as excess red cell production; a condition called polycythemia. Congenital polycythemia can arise due to mutations in EPOR or $p V H L[143,144]$. Acquired polycythemias are classified as primary, when caused by a clonal expansion of hematopoietic stem cells, or secondary, when due to sustained hypoxia caused by several conditions, including high altitude living, chronic lung disease, or EPO injections [145]. Only primary polycythemia vera (PV) will be discussed further. PV has been recognized as a disease entity since the late 1800s, but its etiology remained unknown until a point mutation in the $J A K 2$ gene was shown to cause disease in the great majority of PV patients [146]. This mutation, a substitution of valine to phenylalanine at codon 617 (JAK2V617F), affects a pseudo kinase domain of JAK2 and is believed to impair auto regulation of its kinase activity. The resulting constitutive signaling is thought to mediate the disease phenotype. PV is a clonal disease that arises at the level of multipotent hematopoietic stem cells [147]. Clinically, hallmarks of the disease include elevated red cell counts, splenomegaly, low serum EPO, pruritus, and EPO-independent erythroid colony formation [148]. PV patients are at increased risk of thrombotic events (40\% of cases) and transformation to acute myelocytic leukemia (5$20 \%$ of cases) $[148,149]$. 
Table 1.2 Characteristic CBC findings in anemias of varying etiologies.

\begin{tabular}{|c|c|c|c|c|c|}
\hline & $\begin{array}{l}\text { Normal } \\
\text { value* }\end{array}$ & IDA & AI & $\begin{array}{c}\text { Sideroblastic } \\
\text { Anemia }\end{array}$ & $\begin{array}{c}\text { Megaloblastic } \\
\text { Anemia } \\
\end{array}$ \\
\hline $\begin{array}{c}\text { RBC } \\
(\mathrm{M} / \mu \mathrm{l})\end{array}$ & 5.2 & Low & Low & Low & Low \\
\hline $\begin{array}{l}\text { Het } \\
(\%)\end{array}$ & 47 & Low & Low & Low & Low \\
\hline $\begin{array}{l}\mathbf{H b} \\
(\mathrm{g} / \mathrm{dl})\end{array}$ & 15.5 & Low & Low & Low & Low \\
\hline $\begin{array}{c}\text { MCHC } \\
\text { (g/dl) }\end{array}$ & 34 & Low & Normal & Low & Normal \\
\hline $\begin{array}{l}\text { MCV } \\
\text { (fl) }\end{array}$ & 90 & Low & Normal & Low & High \\
\hline $\begin{array}{c}\text { Reticulocyte } \\
(\mathbf{k} / \mu \mathrm{l})\end{array}$ & $75-100$ & Low & Low & low & Low \\
\hline $\begin{array}{c}\text { Transferrin } \\
\text { Saturation } \\
(\%)\end{array}$ & $20-50$ & Low & Low & High & High \\
\hline
\end{tabular}


Several mouse models expressing $J A K 2 V 617 F$ have been generated, including bone marrow transplant, constitutive knockout in the hematopoietic compartment, floxed mutant allele, and conditional heterozygous or homozygous knock-in models [150-153]. Expression of the mutant allele in mice can also cause essential thrombocythemia (ET) and primary myelofibrosis (PMF), two myeloproliferative diseases related to PV. Patients with ET or PMF can also carry the JAK2V617F mutation, and the exact role of mutant JAK2 as the driver of the disease process remains controversial. It has been suggested that gene dosage could explain the various clinical outcomes seen in patients with this mutation [152]. Other genetic defects associated with PV include mutations in JAK2 exon 12, TET2, IDH1, and IDH2 [154-156].

Treatment options for PV are still limited, despite a recent increased understanding of the molecular pathology. Cytoreductive therapy employing phlebotomy remains the gold standard [148]. This approach, however, carries significant risks of thrombotic events due to a rise in platelet count [157]. Other therapeutic options include 2-hydroxurea and pegylated interferon- $\gamma$. Since the identification of JAK2V617F, multiple JAK2 inhibitors are under development and a handful have been tested in clinical trials [158]. A major challenge with JAK2-targeted therapy is preserving wildtype JAK2 function while targeting the mutant protein. 


\subsubsection{Clinical evidence for synergy between iron and EPO}

The advent of commercially available recombinant human EPO and Erythropoiesis-Stimulating Agents (ESAs) have considerably improved treatment options for patients suffering from anemias due to chronic kidney disease, inflammatory diseases, and malignancy [159]. However, resistance to the pro-erythropoietic effects of ESAs develops in a subset of patients over time [160-162]. Interestingly, concurrent administration of IV iron can restore ESA effectiveness, despite the presence of adequate iron stores. These clinical observations point to a synergistic relationship between iron and EPO in promoting erythroid output.

\subsection{Thesis objectives}

The unique relationship between iron and EPO has long been appreciated, but the molecular mechanisms that optimize red cell production are still poorly understood. The worldwide prevalence of IDA underscores the need for further understanding of erythroid development and identifying new agonists to boost red cell production. Additionally, dissecting the iron-EPO relationship is of great clinical interest in anemias secondary to chronic kidney disease, inflammation, and malignancy, where poor iron utilization impairs the marrow's ability to respond to exogenous EPO. Identification of new targets that integrate iron and EPO-mediated signals could thus lead to new therapeutic options 
for patients with anemias, as well as new means to control red cell production in patients with polycythemias.

Our laboratory has been at the forefront of studying iron-mediated signals that affect erythroid production. We previously showed that the aconitase enzymes are selectively inactivated in erythroid progenitors by low iron levels. The key role of aconitase was confirmed by the ability of its metabolic product, isocitrate, to rescue erythroid differentiation in primary progenitors and correct anemia in iron-deficient mice [87]. This body of work suggested an important role for the aconitase enzymes during steady-state erythropoiesis and raised several new questions. Is aconitase activity required during iron-replete erythropoiesis? How is aconitase activity mechanistically connected to the erythroid differentiation program? Do cytosolic and mitochondrial aconitases play different roles? Intriguingly, our prior studies strongly argued for a nonmetabolic role for aconitase in erythroid differentiation. The central hypothesis of the work presented below is that aconitase enzymes regulate erythropoiesis by interacting with ERK-mediated signaling.

In chapter 2, data will be presented arguing for a positive role for aconitase during erythroid development. Pharmacologic inhibition of aconitase impaired proliferation and differentiation of erythroid progenitors, but spared viability. In mice, aconitase inhibition caused anemia and impaired the response to acute anemic stress. These effects are not mediated by defects in metabolic processes involving aconitase but rather by interactions with discrete ERK signaling events. Chapter 3 will provide additional evidence regarding the role of ERK1/2 as a mediator of aconitase and address a possible role for 
ERK-directed RSK signaling. Data will also be presented highlighting important differences between the effects of iron deprivation and aconitase blockade on erythropoiesis. Collectively, these results suggest that aconitase enzymes function as integrators of EPO signals and iron status. Manipulation of this pathway may have therapeutic benefits in disorders of erythroid production. 


\section{CHAPTER 2:}

\section{Aconitase Regulation of Erythropoiesis Correlates with a Novel Licensing Function in Erythropoietin-induced ERK Signaling}

Modified from the version published in PLOS ONE:

Talbot AL, Bullock, GC, Delehanty LL, Sattler M, Zhao ZJ, Goldfarb AN. Aconitase

Regulation of Erythropoiesis Correlates with a Novel Licensing Function in Erythropoietin-induced ERK Signaling. PLoS ONE. 2011 August 22. 


\section{Abstract}

Erythroid development requires the action of erythropoietin on committed progenitors to match red cell output to demand. In this process, iron acts as a critical cofactor, with iron deficiency blunting EPO-responsiveness of erythroid progenitors. Aconitase enzymes have recently been identified as possible signal integration elements that couple erythropoiesis with iron availability. In the current study, a regulatory role for aconitase during erythropoiesis was ascertained using a direct inhibitory strategy. In C57BL/6 mice, infusion of an aconitase active-site inhibitor caused a hypoplastic anemia and suppressed responsiveness to hemolytic challenge. In a murine model of polycythemia vera, aconitase inhibition rapidly normalized red cell counts, but did not perturb other lineages. In primary erythroid progenitor cultures, aconitase inhibition impaired proliferation and maturation but had no effect on viability or ATP levels. This inhibition correlated with a blockade in EPO signal transmission specifically via ERK, with preservation of JAK2-STAT5 and Akt activation. Correspondingly, a physical interaction between ERK and mitochondrial aconitase was identified and found to be sensitive to aconitase inhibition. Direct aconitase inhibition interferes with erythropoiesis in vivo and in vitro, confirming a lineage-selective regulatory role involving its enzymatic activity. This inhibition spares metabolic function but impedes EPO-induced ERK signaling and disturbs a newly identified ERK-aconitase physical interaction. We propose a model in which aconitase functions as a licensing factor in ERK-dependent proliferation and differentiation, thereby providing a regulatory input for iron in EPO- 
dependent erythropoiesis. Directly targeting aconitase may provide an alternative to phlebotomy in the treatment of polycythemia vera. 


\section{Introduction}

Production of red blood cells, or erythropoiesis, is regulated by the cytokine erythropoietin (EPO) in conjunction with iron. In the context of an adequate iron supply, EPO promotes proliferation, differentiation, and survival of erythroid progenitors, beginning at the colony forming unit-erythroid stage. Iron restriction, i.e. diminished amounts of bio-available iron, results in diminished red cell production due to decreased erythroid proliferation and maturation [85]. Iron regulation of erythropoiesis has been documented in many experimental models including rats, where iron deficiency causes a defect in the transition from the CFU-e stage to the proerythroblast stage [86]. Iron modulation of EPO bioactivity occurs clinically in patients whose response to recombinant EPO can be augmented by exogenous iron despite adequate iron stores [160]. Thus, iron sensing mechanisms in the erythroid compartment function in a rheostatic manner to adjust output based on iron availability.

Aconitase enzymes have recently been identified as mediators of the erythroid response to iron restriction [87]. In mammals, aconitases consist of mitochondrial and cytosolic isoenzymes that both utilize a prosthetic $4 \mathrm{Fe}-4 \mathrm{~S}$ cubane iron-sulfur cluster group at their active site to interconvert the metabolites citrate and isocitrate [106]. They are highly sensitive to intracellular iron levels and redox conditions. Cellular iron deprivation causes loss of the $\alpha-\mathrm{Fe}^{2+}$ group from the iron-sulfur cluster, while oxidative stress induces complete cluster disassembly, both of which conditions inactivate enzymatic function $[111,163]$. An additional level of enzymatic regulation may arise 
from phosphorylation $[112,164]$. Both isoforms also exert non-enzymatic functions through interaction with nucleic acid targets. Cytosolic aconitase, in its role as Iron Regulatory Protein 1, binds iron response elements within mRNA sequences of a cohort of iron responsive genes and regulates their expression [124]. Mitochondrial aconitase contributes to the protein complexes assembled with the mitochondrial genome and participates in mitochondrial DNA maintenance [135].

EPO engagement of its receptor activates the associated cytosolic tyrosine kinase JAK2, which in turn activates multiple signal transduction pathways critical in erythropoiesis. One such pathway consists of Raf-MEK-ERK [165-167]. In many cell types, ERK activation exerts both positive and negative effects on proliferation and differentiation, with signal output determined by kinetics and magnitude of activation, subcellular localization, scaffolds, and crosstalk with other signaling modules [70]. Known subcellular locations for ERK include the plasma membrane, endosomes, Golgi apparatus, nucleus, and mitochondria [168-172]. With regard to erythropoiesis, enforced ERK activation by mutant N-Ras V12 expression in ex vivo murine fetal liver erythroblast cultures blocked differentiation and promoted proliferation [46]. In vivo studies also suggest that ERK1 signaling negatively regulates red cell production. In these studies, ERK1 null mice displayed a higher basal rate of splenic erythropoiesis and responded more rapidly to hemolytic challenge [68]. On the other hand, MEK inhibition strongly diminished the yield of erythroid colonies from wild type murine marrow cultured in the presence of EPO [66]. Thus, as with many cell types, ERK signaling exerts both positive and negative effects during erythropoiesis. 
Iron regulation of erythropoiesis appears to involve aconitase-mediated alterations in EPO signal transduction and cellular metabolism [87]. Because iron deprivation affects numerous cellular pathways in addition to aconitase, the current studies have employed a targeted inhibitory approach to more specifically define the role of aconitase activity in erythropoiesis. These studies, using a potent active-site inhibitor, revealed that aconitase activity regulates in vivo steady-state erythropoiesis, as well as responsiveness to acute anemia. Ex vivo studies confirmed this regulatory relationship, with aconitase inhibition affecting proliferation and differentiation but not viability. Surprisingly, doses of the aconitase inhibitor that effectively inhibited erythropoiesis had no effects on cellular ATP levels or on EPO induction of JAK2 activation but did block ERK-mediated phosphorylation of RSK substrates. A physical interaction between aconitase and ERK was observed and found to be sensitive to the inhibitor.

As a putative mediator of the erythroid response to iron restriction, aconitase activity could offer a target for controlling disease in patients with polycythemia vera. PV is a hematopoietic stem cell disorder associated with an activating mutation in JAK2 $[147,173]$ that causes expansion of red cell mass and possible thrombotic and hemorrhagic complications [149]. The treatment of choice for normalizing red cell mass has consisted of induction of iron deficiency through repeated phlebotomy. In this study, low-dose infusion of the aconitase inhibitor rapidly normalized red cell counts in a murine model of PV. Thus the novel pathway for aconitase regulation of erythropoiesis described herein bears potential for therapeutic manipulation in the setting of oncogenic signaling. 


\section{Results}

\section{Induction of anemia by the aconitase inhibitor fluoroacetate}

Aconitase enzymes have recently been implicated in the erythroid iron restriction response in ex vivo progenitor cultures and in iron deficient mice [87]. To define the contribution of aconitase enzymatic activity to erythropoiesis in vivo, the potent and specific active-site inhibitor of aconitase fluoroacetate (FA) was administered to mice [117]. Previous studies have shown that FA can be safely administered to mice at a dose of $4 \mathrm{mg} / \mathrm{kg} / \mathrm{day}$ using a continuous infusion pump, a regimen that causes a $60 \%$ increase in resting serum citrate levels [174]. Accordingly, an infusion of FA or $0.9 \%$ saline was delivered to C57BL/6 mice at this dosing rate [174]. Complete blood counts obtained after 14 days revealed anemia in the FA-treated animals compared with controls (Table 2.1). In particular, red blood cell (RBC) counts, hemoglobin, and hematocrit were significantly decreased in the FA-treated group. Importantly, the mean corpuscular hemoglobin concentration (MCHC) was unaffected by aconitase inhibition (Table 1), in contrast to the hypochromic anemias associated with defective heme biosynthesis (see Discussion). With regard to non-erythroid cells, FA treatment at $4 \mathrm{mg} / \mathrm{kg} /$ day caused a slight decrease in absolute neutrophil counts (ANC) and did not affect platelets (Table 1). The inhibition of the ANC was not reproducible in subsequent experiments. By contrast, the erythroid abnormalities associated with FA infusion were highly reproducible in 
Table 2.1 Hematologic parameters of C57BL/6 mice treated for 14 days with saline $(0.9 \%)$ or FA (4 mg/kg/day).

\begin{tabular}{lccc}
\hline Blood parameter & $\begin{array}{c}\text { Saline } \\
(\mathbf{n = 1 0})\end{array}$ & $\begin{array}{c}\text { FA-treated } \\
(\mathbf{n}=\mathbf{1 0})\end{array}$ & $\begin{array}{c}\boldsymbol{P} \\
\text { value }\end{array}$ \\
\hline RBC, $\mathbf{~ 1 0} / \mu \mathbf{L}$ & $9.32 \pm 0.7$ & $7.45 \pm 0.09$ & $<0.001^{*}$ \\
Hematocrit, \% & $44.0 \pm 3.5$ & $36.0 \pm 4.4$ & $<0.005^{*}$ \\
Hemoglobin, g/dL & $14.7 \pm 1.08$ & $11.6 \pm 1.0$ & $<0.001^{*}$ \\
MCV, fL & $47.24 \pm 0.71$ & $48.49 \pm 0.7$ & $0.01^{*}$ \\
MCHC, g/dL & $33.6 \pm 2.76$ & $32.41 \pm 3.10$ & 0.38 \\
Platelets, $\mathbf{~} \mathbf{1 0} / \mu \mathbf{L}$ & $804 \pm 84$ & $734 \pm 7.3$ & 0.067 \\
Neutrophils, $\mathbf{~} \mathbf{1 0} / \mu \mathrm{L}$ & $1.28 \pm 0.3$ & $0.89 \pm 0.4$ & $0.02^{*}$ \\
\hline
\end{tabular}

RBC indicates Red Blood Cell; MCV, Mean Corpuscular

Volume; MCHC, Mean Corpuscular Hemoglobin Concentration.

Data are presented as mean \pm SD.

* significantly different from value for saline-treated mice. 
multiple experiments; even those using a $2 \mathrm{mg} / \mathrm{kg} /$ day dosing regimen (see Table 2.2). To confirm that FA treatment inhibited aconitase activity in vivo, serum citrate levels were measured, and the $2 \mathrm{mg} / \mathrm{kg} /$ day dosing regimen was found to cause a 1.9 -fold increase in mean serum citrate concentration (Figure 2.1).

Further characterization revealed the anemia to be associated with a 2.5 -fold increase in serum EPO levels (Figure 2.2A) and a significant decrease in absolute reticulocyte counts (Figure 2.2B). Flow cytometric analysis of bone marrow erythroid maturation employed co-staining for CD71 and Ter119, as has been described (Figure 2.2 C) [26]. The FA-treated mice showed a significant increase in frequency and absolute number of R3 erythroid precursors (CD71 $1^{\text {Intermediate }}$ Ter119 $9^{\text {Bright }}$ ) (Figure 2.2.C-E). This defect in erythroid maturation was observed in multiple independent experiments involving in vivo aconitase inhibition. Maturation was also analyzed using a recently described approach that resolves Ter $119^{+}$erythroid precursors into five distinct subpopulations based on CD44 levels and forward scatter (FSC) [175]. This approach showed a significant increase in the frequency of $\mathrm{CD}^{-} 4^{-} \mathrm{FSC}^{\text {low }}$ cells (stage V) associated with FA treatment (Figure 2.3). 
Figure 2.1 Elevated serum citrate levels in mice treated with fluoroacetate. Sera from saline-treated and FA-treated ( $2 \mathrm{mg} / \mathrm{kg} /$ day $)$ mice were isolated from whole blood collected on day 26 post initiation of treatment. Citrate levels were determined enzymatically. Data are presented as mean $\pm \mathrm{SD} ; \mathrm{n}=8$ per group; ${ }^{\ddagger} P<0.001$. 
Figure 2.1 Elevated serum citrate levels in mice treated with fluoroacetate.

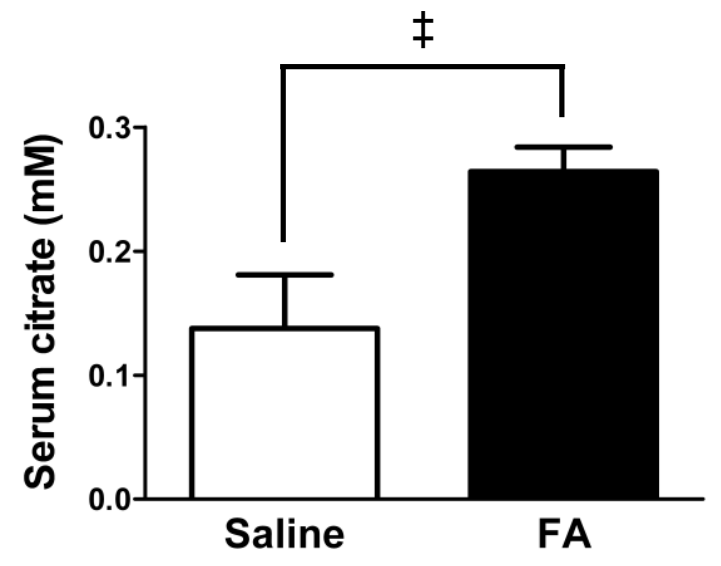


Figure 2.2 In vivo effects of aconitase inhibition. (A) Serum EPO levels in mice treated for 14 days with an infusion of saline $(0.9 \%)$ or FA (4 mg/kg/day). Data represent mean $\pm \mathrm{SD} ; \mathrm{n}=9$ for saline group and 10 for FA group; ${ }^{\ddagger} P<0.001$. (B) Absolute reticulocyte counts in mice treated as in panel $\mathbf{A}$. Data represent mean $\pm \mathrm{SD} ; \mathrm{n}=10$ per group; ${ }^{\ddagger} P<0.001$. (C) Erythroid maturation in marrows from mice who received a fourweek infusion of FA (2 mg/kg/day) or saline ( $0.9 \%)$. Representative flow cytometry plots are shown. Boxes indicate erythroid developmental stages R1-R4, from top left (R1) to bottom right (R4). (D), (E) Summary of data from panel C displayed as cell percentages (D) or absolute cell numbers per femur $(\mathbf{E})$ in regions R1-R4. Data is presented as mean $\pm \mathrm{SD} ; \mathrm{n}=4$ per group; ${ }^{*} P<0.05$. 
Figure 2.2 In vivo effects of aconitase inhibition.
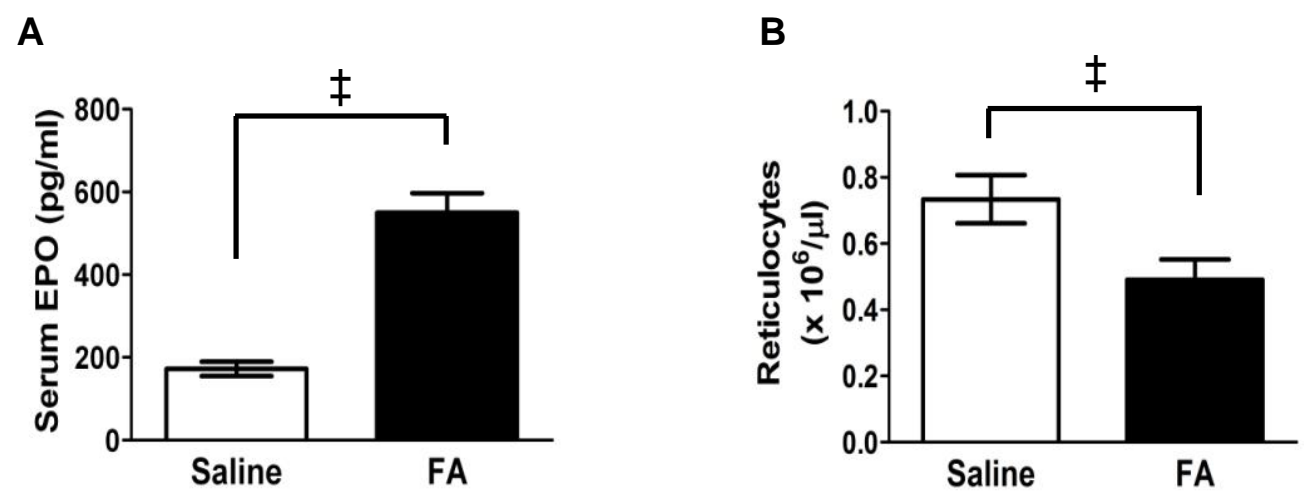

C

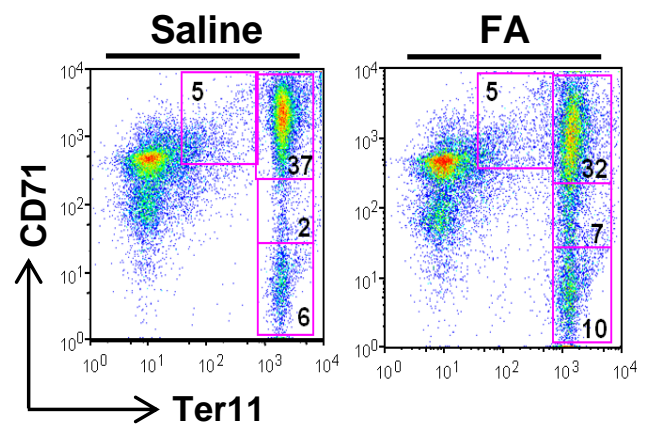

D

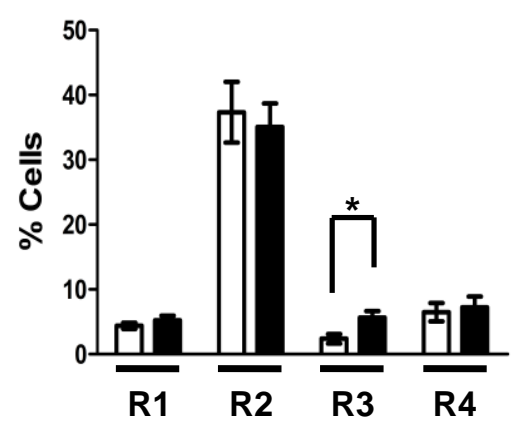

E

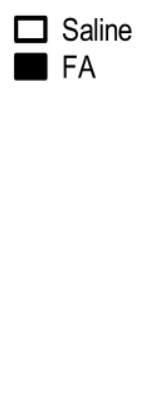

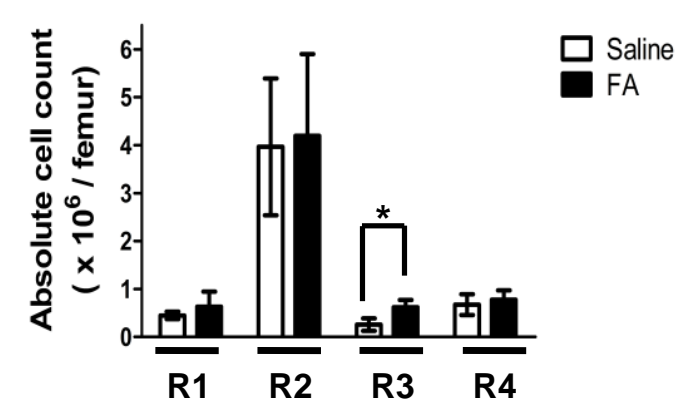


Figure 2.3 Analysis of the bone marrow erythroid maturation using Ter119 and CD44.

(A) Erythroid maturation in marrows from mice who received a four-week infusion of FA $(2 \mathrm{mg} / \mathrm{kg} / \mathrm{day})$ or saline $(0.9 \%)$. After gating on the Ter $119^{+}$fraction, cells were analyzed for CD44 expression versus Forward Scatter (FSC). Representative flow cytometry plots are shown. Roman numerals I-V indicate distinct erythroid subpopulations. (B) Summary of data from panel A with mean percentages of cells in subpopulations I-V $\pm \mathrm{SD} ; \mathrm{n}=4$ per group; ${ }^{*} P<0.05$. (C) Summary of data from panel A with absolute number of cells per femur in subpopulations $\mathrm{I}-\mathrm{V} \pm \mathrm{SD} ; \mathrm{n}=4$ per group; ${ }^{\dagger}$ $P<0.01$ 
Figure 2.3 Analysis of the bone marrow erythroid maturation using Ter119 and CD44.

$\mathbf{A}$

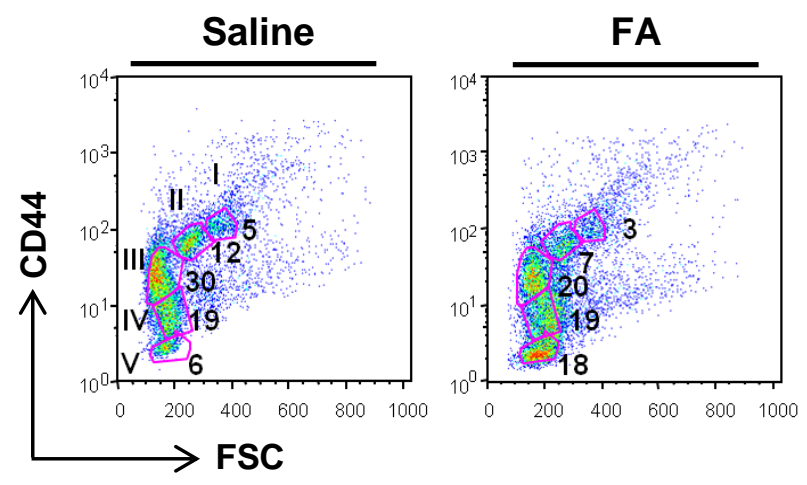

B

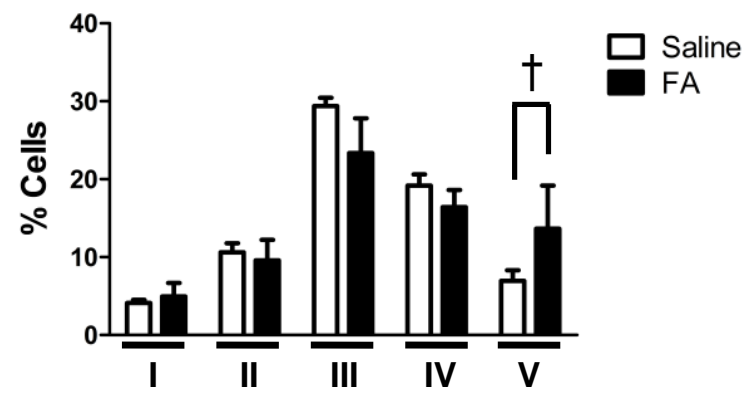

C

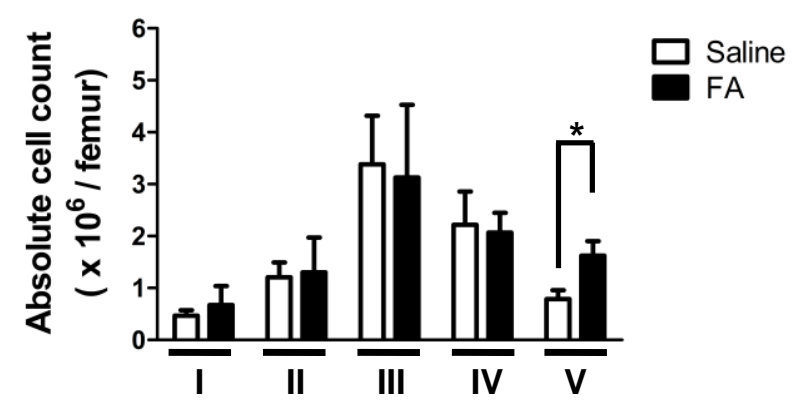




\section{Aconitase inhibition impairs responsiveness to anemic challenge}

Erythropoiesis can increase dramatically in response to acute anemia. To determine whether aconitase inhibition interferes with the capacity to respond to anemic challenge, mice treated with saline or $4 \mathrm{mg} / \mathrm{kg} / \mathrm{day}$ FA received intraperitoneal injections of the hemolytic agent phenylhydrazine (PHZ) with dosage reduction made for the FAtreated group to prevent lethality (see Materials and Methods). PHZ treatment typically leads to an RBC nadir on day 3 post-injection followed by complete recovery on day 7 [59]. Despite receiving half the standard PHZ dose, mice treated with FA developed an anemia that was much more pronounced and prolonged than that of the control mice. Specifically, their RBC counts reached nadir on day 7 instead of day 3 and did not return to baseline even 17 days after PHZ administration (Figure 2.4A). In addition, their reticulocyte response was delayed and diminished (Figure 2.4B).

Further characterization of the role of aconitase in the response to acute anemia employed mice lacking cytosolic aconitase (IRP1 -/- mice) [130]. These mice, along with wild-type aged-matched controls, received standard doses of PHZ and were monitored for responsiveness. As shown in Figures 2.4C and 2.4D, IRP1 -/- mice responded to PHZ-induced anemia in a manner indistinguishable from wild type mice. The starting RBC counts, the magnitude of their decrease, and the rate of recovery were not significantly different in the two groups; both also exhibited a similar reticulocyte response during the recovery phase. These results indicate that cytosolic 
Figure 2.4 Inhibition of aconitase impairs responsiveness to anemic challenge. (A) RBC and (B) absolute reticulocyte counts in PHZ-challenged mice treated with either FA $(4 \mathrm{mg} / \mathrm{kg} / \mathrm{day} ; \mathrm{n}=11)$ or saline $(0.9 \% ; \mathrm{n}=6)$. PHZ injections on days 0 and 1 consisted of $30 \mathrm{mg} / \mathrm{kg} /$ day for the FA group and $60 \mathrm{mg} / \mathrm{kg} /$ day for the saline group. Data represent mean \pm SD. (C) RBC and (D) absolute reticulocyte counts in IRP1 -/- and wild type (WT) mice. PHZ doses for both groups consisted of $60 \mathrm{mg} / \mathrm{kg} /$ day on days 0 and 1 . Data represent mean $\pm \mathrm{SD} ; \mathrm{n}=8$ per group. 
Figure 2.4 Inhibition of aconitase impairs responsiveness to anemic challenge.

A

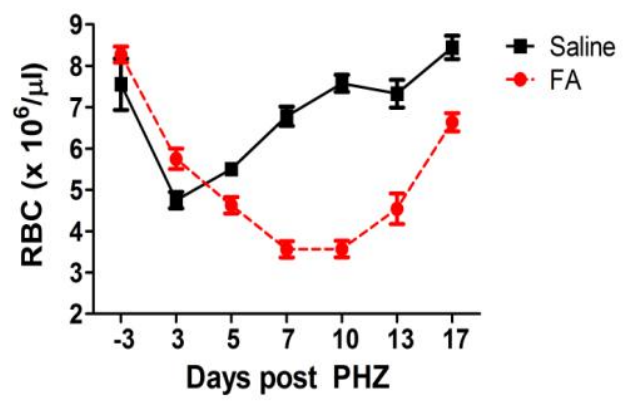

C

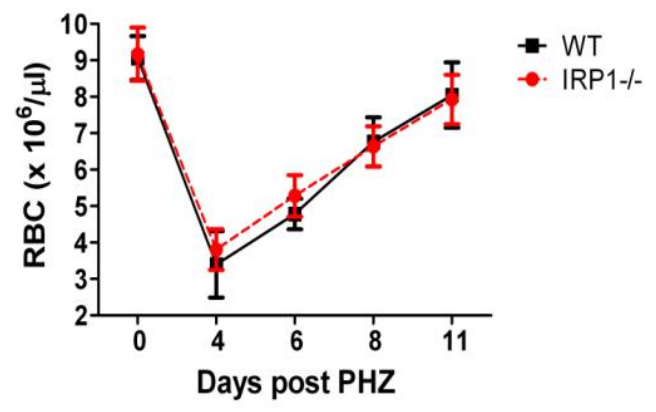

B

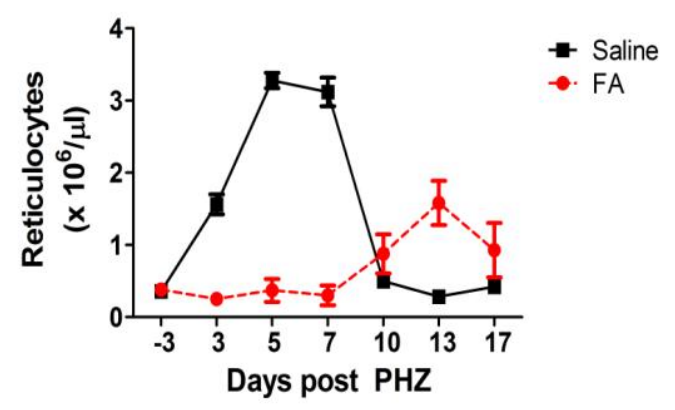

D

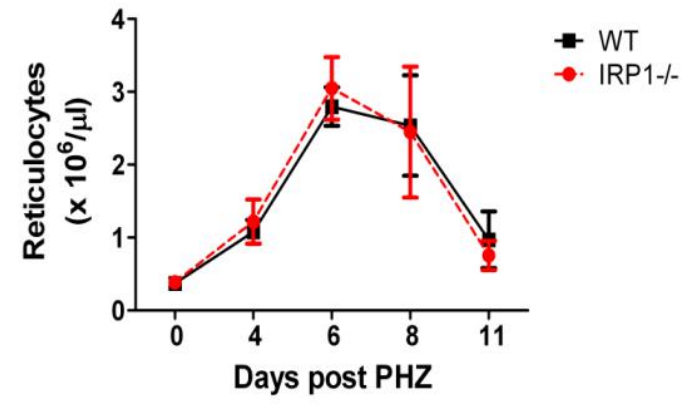


aconitase is dispensable for both steady-state erythropoiesis and for responsiveness to acute anemia (see Discussion).

\section{Aconitase inhibition affects erythropoiesis in a cell-intrinsic manner}

The in vivo effects of FA infusion support a role for aconitase activity in the regulation of erythropoiesis but could also reflect indirect influences mediated by the marrow microenvironment. Therefore, ex vivo primary cultures were used to examine the effects of aconitase inhibition directly on erythropoiesis. In these experiments, purified human $\mathrm{CD} 34^{+}$cells were cultured in unilineage erythroid medium (see Materials and Methods) with or without fluoroacetate. Aconitase inhibition caused a dosedependent decrease in proliferation over a five day culture period, with $10 \mu \mathrm{M}$ causing a statistically significant decrease and $100 \mu \mathrm{M}$ abrogating growth altogether (Figure 2.5A). The proliferation defect was not secondary to cell death, as FA treatment had minimal effects on overall viability and no effects on apoptotic signaling (Figure 2.5B-C). All subsequent experiments employed $50 \mu \mathrm{M}$ FA because this dose reproducibly inhibited proliferation and maturation (see below) without affecting viability. In addition, this dose strongly inhibited aconitase activity in erythroid progenitors, causing a 10-fold increase in intracellular citrate levels (Figure 2.5D).

Erythroid differentiation was assessed by examining the expression of two developmental markers: CD34, a stem cell antigen rapidly downregulated after the early BFU-e stage, and glycophorin A (GPA), an erythroid- specific antigen appearing 
Figure 2.5 Effects of aconitase inhibition on erythroid proliferation, survival and maturation in vitro. (A) Fold increase in number of primary human progenitors cultured for five days in erythroid medium with the indicated doses of FA. Shown are mean \pm SD for three independent experiments; ${ }^{*} P<0.05 ;{ }^{\dagger} P<0.01$. (B) Survival of cells from day 5 erythroid cultures $\pm 50 \mu \mathrm{M}$ FA assessed by staining with 7-amino actinomycin D (7-AAD) and annexin V-PE followed by flow cytometry; numbers indicate percentages of cells in each quadrant. (C) Apoptotic signaling in cells from day 5 erythroid cultures with $0,10,50$, and $100 \mu \mathrm{M}$ FA assessed by immunoblotting of whole cell lysates for caspase-3 (arrow indicates position of cleavage product). (D) Increased intracellular citrate in FA-treated cells. $\mathrm{CD} 34^{+}$cells were cultured in erythroid medium for 5 days $\pm 50 \mu \mathrm{M}$ FA. Cellular extracts were assayed for citrate levels enzymatically. Data are presented as mean fold increase over control cells $\pm \mathrm{SD} ; \mathrm{n}=4 ;^{\dagger} P<0.01$. (E) Differentiation of erythroid progenitors cultured as in panel C. Surface expression of CD34 and GPA was assessed by flow cytometry on live cells; numbers indicate percentages of cells in each quadrant. (F) Multiple independent experiments as in panel E show an increase in the percentage of $\mathrm{CD} 34^{+} \mathrm{GPA}^{+}$cells associated with FA treatment. Shown are mean $\pm \mathrm{SD} ; \mathrm{n}=6 ;{ }^{\star} P<0.001$. 
Figure 2.5 Effects of aconitase inhibition on erythroid proliferation, survival and maturation in vitro.

A

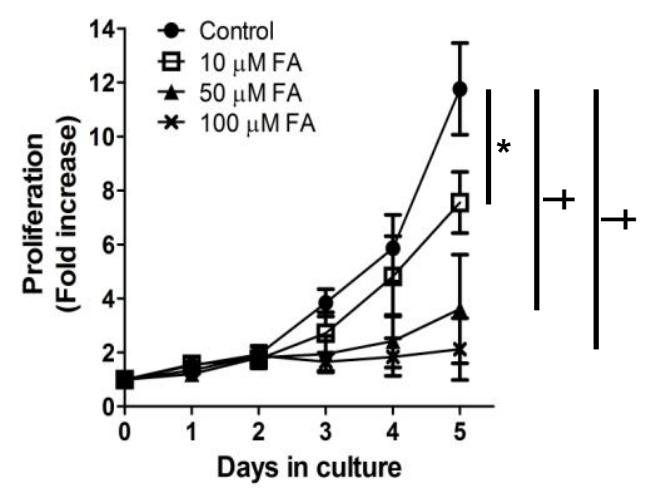

C

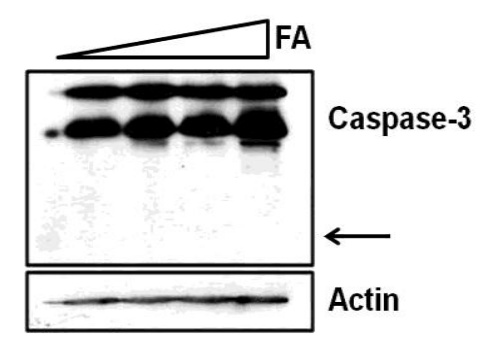

$\mathbf{E}$

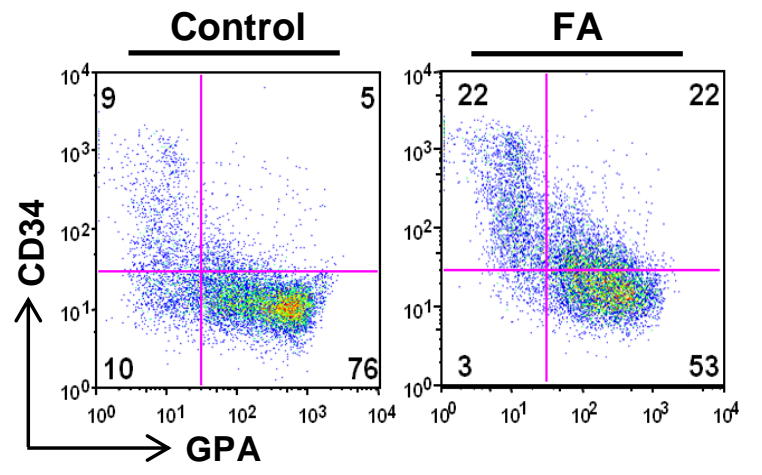

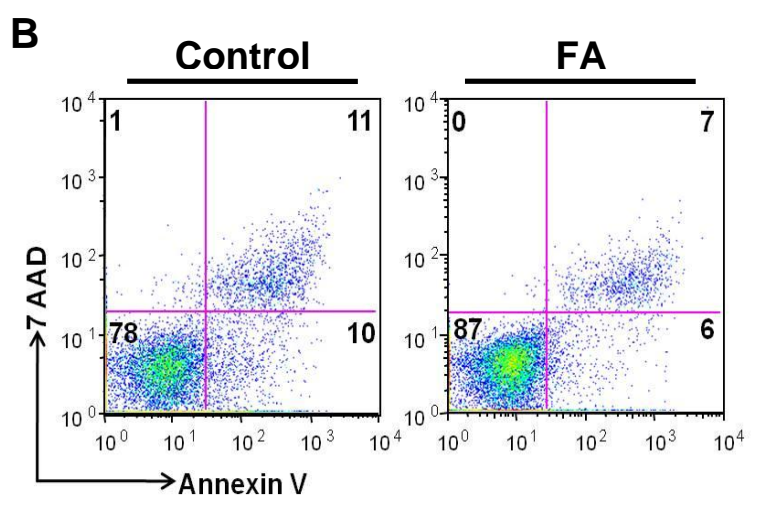

D

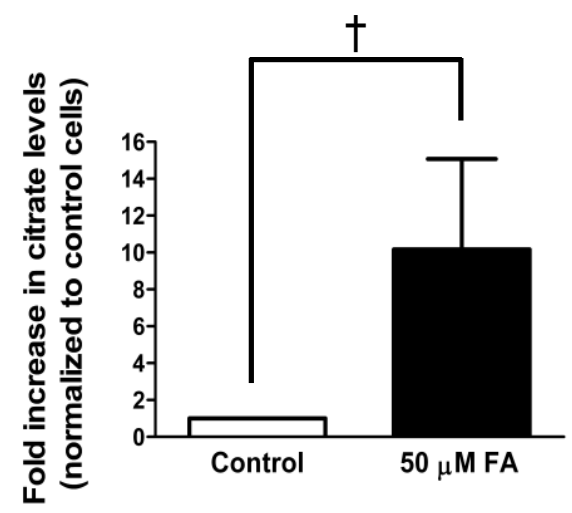

F

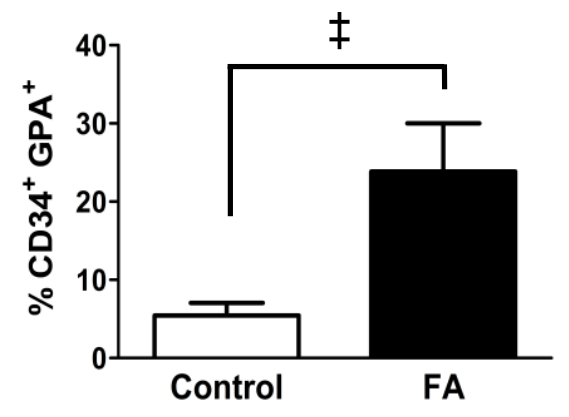


initially at the proerythroblastic stage and continually increasing with differentiation $[25,176]$. Addition of FA to the erythroid cultures impaired the downregulation of CD34 while attenuating the upregulation of GPA (Figure 2.5E). Specifically, FA treatment caused a 5-fold increase in the frequency of cells coexpressing CD34 and GPA, two markers that minimally overlap during normal maturation (Figure 2.5F). These results demonstrate cell-intrinsic defects in proliferation and maturation associated with aconitase inhibition in cultured erythroid progenitors.

\section{The effects of aconitase inhibition on erythropoiesis occur independently of metabolic impairment}

To determine whether the defect in erythropoiesis caused by aconitase inhibition occurred secondary to alterations in Krebs cycle metabolism or mitochondrial dysfunction, several parameters were assessed. Firstly, $50 \mu \mathrm{M}$ FA had no effect on cellular steady state ATP levels in day 5 erythroid cultures (Figure 2.6A). Secondly, FA treatment did not alter the activation status of AMP-kinase (AMPK) (Figure 2.6B). AMPK functions as a sensor of the AMP:ATP ratio; an increase in this ratio activates AMPK, which in turn upregulates catabolic pathways to restore cellular ATP levels [177]. Thirdly, the FA treatment did not alter levels of reactive oxygen species, as detected by the fluorescent indicator 3'-(aminophenyl)-fluorescein (APF) [178]. APF mean fluorescence intensity (MFI) was identical in untreated and FA-treated erythroid cultures, both in early (GPA negative gate) and late progenitors (GPA positive gate) 
Figure 2.6 FA inhibits erythropoiesis in the absence of changes in cellular ATP, ROS, or MMP. (A) ATP levels in cells from day 5 erythroid cultures of human CD34 ${ }^{+}$ progenitors treated with $0-100 \mu \mathrm{M}$ FA. Shown are values relative to untreated cultures consisting of mean \pm SD from three independent experiments. (B) APMK activation in cells from day 5 erythroid cultures with $0-100 \mu \mathrm{M}$ FA assessed by immunoblotting of whole cell lysates for phospho- and total AMPK. (C) Levels of reactive oxygen species (ROS) in cells from day 3 erythroid cultures $\pm 50 \mu \mathrm{M}$ FA detected by the fluorescent probe APF. Mean fluorescence intensities (Geom. Mean) of the APF signal within GPAnegative and positive gates are shown adjacent to flow cytometry plots. (D) Mitochondrial membrane potential in cells from day 3 erythroid cultures $\pm 50 \mu \mathrm{M}$ FA detected by MitoTracker CMXRos. Mean fluorescence intensities (Geom. Mean) of the MitoTracker signal within GPA-negative and positive gates are shown adjacent to flow cytometry plots. 
Figure 2.6 FA inhibits erythropoiesis in the absence of changes in cellular ATP, ROS, or MMP.

A

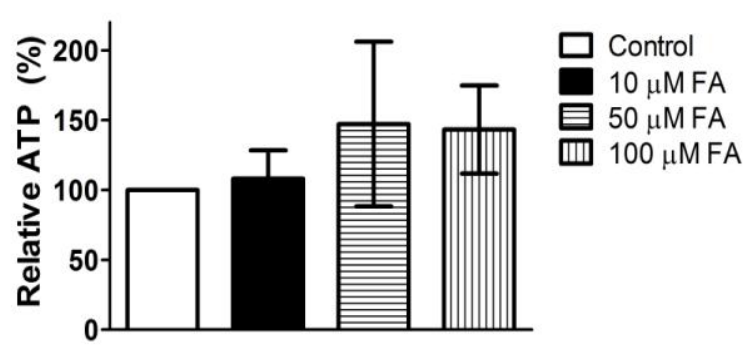

B

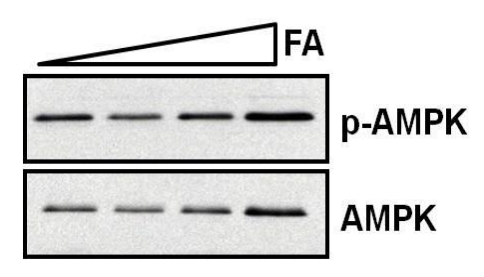

C
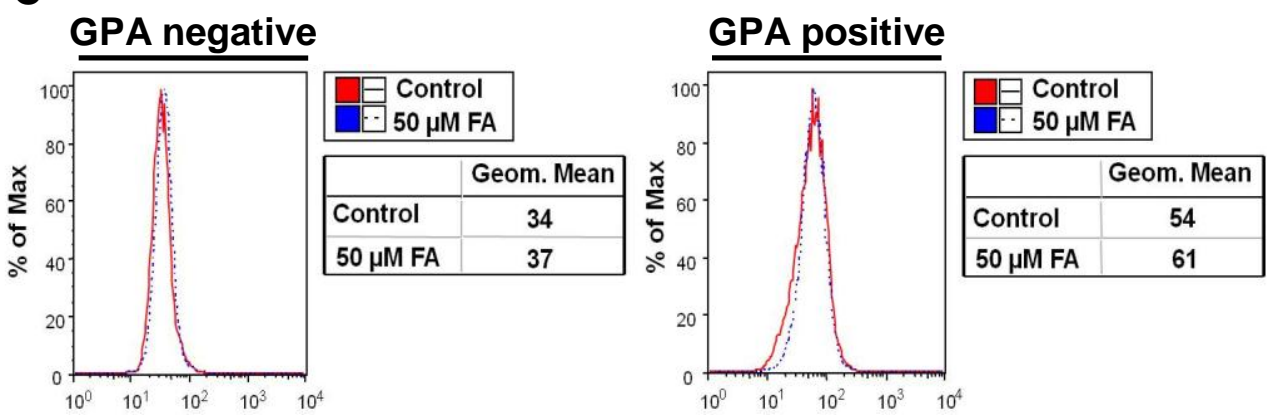

D
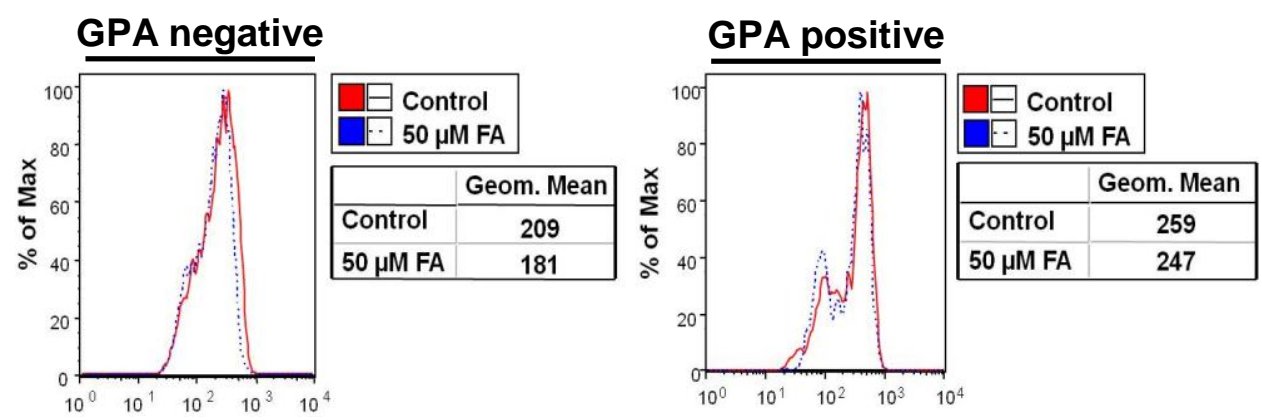
(Figure 2.6C). Fourthly, $50 \mu \mathrm{M}$ FA did not alter the mitochondrial membrane potential (MMP), as assessed by staining with the membrane permeant dye MitoTracker CMXRos [179]. In both early (GPA negative) and more mature (GPA positive) populations, the MFI of MitoTracker staining was unaffected by FA treatment (Figure 2.6D). These results demonstrate that the degree of aconitase inhibition sufficient to impair erythropoiesis is below the threshold that disrupts cellular ATP levels, redox status, or MMP.

\section{Aconitase modulates ERK signaling downstream of EPO}

Several studies have identified non-metabolic functions for aconitase, including participation in signaling modules $[87,111,112]$. Therefore experiments addressed whether aconitase inhibition affected the activation state of EPO-dependent signaling pathways. Immunoblotting with phospho-specific antibodies on whole cell lysates from erythroid cultures showed no effects of FA on basal signaling via JAK2-STAT5, calcineurin-NFAT, PI3K-Akt, $\mathrm{PKC}_{\alpha / \beta I I}$, and PLC $\gamma$ pathways (data not shown) $[55,180$ 183]. However, aconitase inhibition correlated with increased steady state levels of phosphorylated ERK (Figure 2.7A). Unexpectedly, this increase in phospho-ERK was accompanied by a decrease in phosphorylation of p90 ribosomal S6 kinase (RSK) on an ERK target site, threonine 573 [184]. 
Figure 2.7 Aconitase inhibition interferes with EPO activation of ERK signaling in a pathway-specific manner. (A) Steady state phosphorylation of ERK and RSK as determined by immunoblotting of whole cell lysates of cells from days 3-4 erythroid cultures treated with $50 \mu \mathrm{M}$ FA and $10 \mu \mathrm{M}$ U0126 as indicated (NT, untreated). (B) Time course of ERK1/2 and RSK phosphorylation. Cells from day 3 erythroid cultures \pm $50 \mu \mathrm{M}$ FA were subjected to 3 hours of cytokine starvation followed by stimulation with 4.5 U/ml EPO for the indicated time periods. (C) EPO induction of multiple downstream pathways in cells treated as in panel B. (D) Densitometric analysis of pERK1/2:ERK1/2 and pRSK:RSK levels in five independent experiments conducted as in panel $\mathbf{C}$. Presented are mean values \pm SEM for relative phosphoprotein signal divided by total protein signal; $* P<0.05 ;{ }^{\dagger} P<0.01$; n.s., not significant. 
Figure 2.7 Aconitase inhibition interferes with EPO activation of ERK signaling in a pathway-specific manner.

A

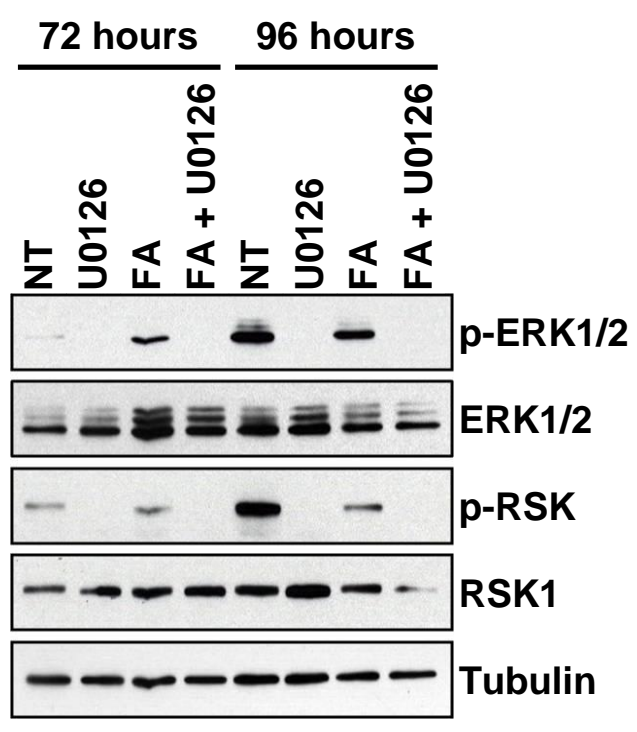

C

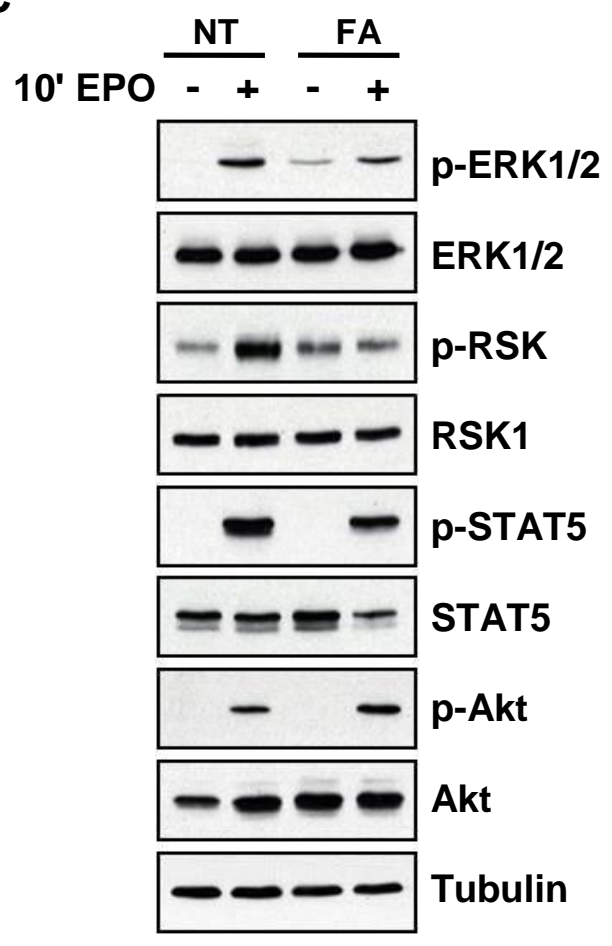

B

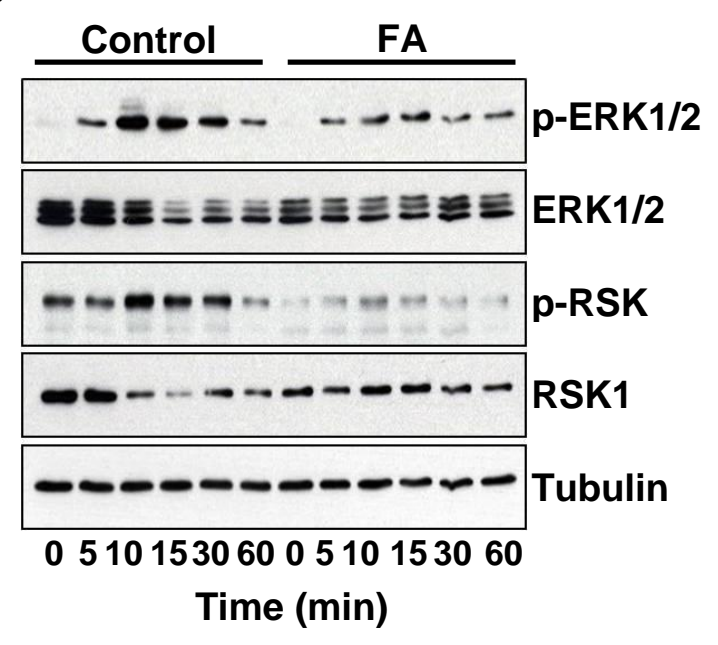

D
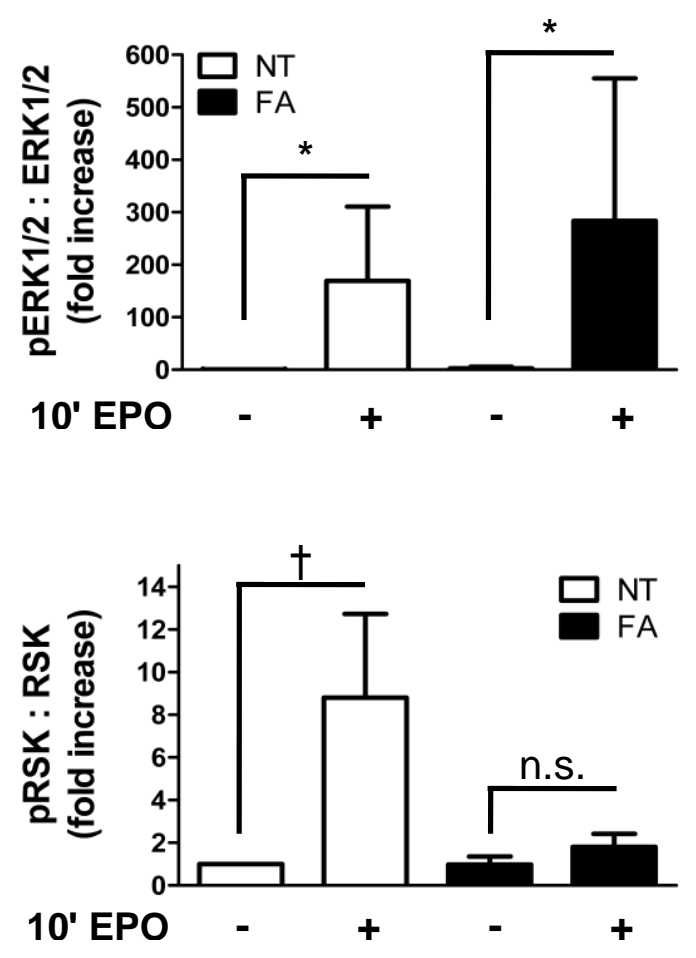
The requirement of MEK-ERK signaling for RSK T573 phosphorylation was confirmed by treatment of cells with the MEK inhibitor U0126 (Figure 2.7A).

To characterize the effects of aconitase inhibition on the initiation of EPO signaling, erythroid progenitors pre-cultured with or without FA underwent cytokine starvation followed by acute stimulation with EPO. FA treatment did not prevent EPO activation of ERK but almost completely blocked its activation of RSK (Figure 2.7B). In control cells treated with EPO, phospho-ERK levels peaked at 10 minutes, and phosphoRSK levels peaked at 15 minutes (Figure 2.7B). In the FA treated cells, EPO induction of phospho-ERK also peaked at 10 minutes, but phospho-RSK showed no increase over the entire 1 hour time course. These signaling abnormalities were consistently observed in multiple independent experiments (Fig. 2.7D). By contrast, FA pretreatment had no effect on EPO induction of STAT5 or Akt activation (Figure 2.7C). The data from steady state erythroid cultures and from EPO stimulation of cytokine-starved cells thus show that aconitase inhibition interferes with ERK-mediated activation of RSK.

\section{MEK inhibition recapitulates the effects of aconitase inhibition on erythropoiesis}

To determine whether impaired ERK signaling might underlie the erythroid defects associated with aconitase inhibition, FA and the MEK inhibitor U0126 were compared for their effects on erythropoiesis as in Figure 2.5. The MEK inhibitor at 10 $\mu \mathrm{M}$ reproducibly inhibited proliferation-to the same degree as $50 \mu \mathrm{M}$ FA (Figure 2.8A), and both agents yielded similar cell cycle perturbations on propidium iodide staining, i.e. 
Figure 2.8 The effects of MEK inhibition on erythropoiesis recapitulate those of aconitase inhibition. (A) Fold increase in number of primary progenitors cultured for 3 days with either $50 \mu \mathrm{M} F A$ or $10 \mu \mathrm{M}$ U0126. Shown are mean \pm SD for three independent experiments; ${ }^{\dagger} P<0.01$. (B) Cell cycle profiles of cells from day 3 cultures as in panel A determined by propidium iodide staining followed by flow cytometry. Percentages of cells in $\mathrm{G}_{0} / \mathrm{G}_{1}, \mathrm{~S}$, and $\mathrm{G}_{2} / \mathrm{M}$ phases are indicated. (C) Multiple independent experiments as in panel $\mathbf{B}$ showing an increase in the percentage of cells in $\mathrm{G}_{0} / \mathrm{G}_{1}$ associated with either FA or U0126 treatment. Data represent mean $\pm \mathrm{SD} ; \mathrm{n}=3 ;^{\dagger}$ $P<0.01$. (D) Erythroid differentiation of progenitors cultured as in panel A assessed by staining for GPA and CD34 followed by flow cytometry; percentages of cells in each quadrant are shown. (E) Viable fractions from panel D determined by forward (FSC) and side (SSC) light scatter properties. 
Figure 2.8 The effects of MEK inhibition on erythropoiesis recapitulate those of aconitase inhibition.

A

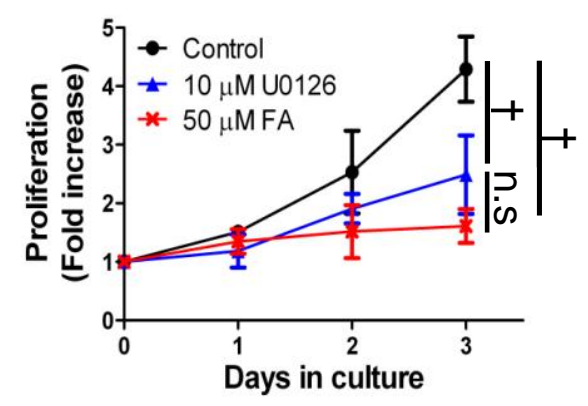

B

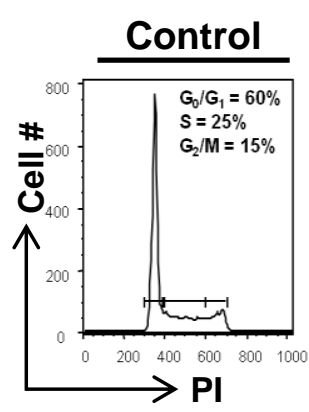

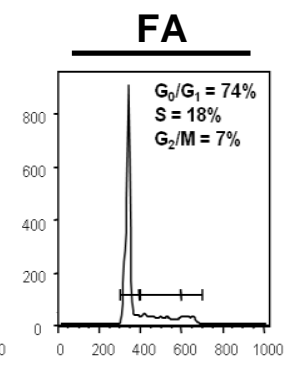

C

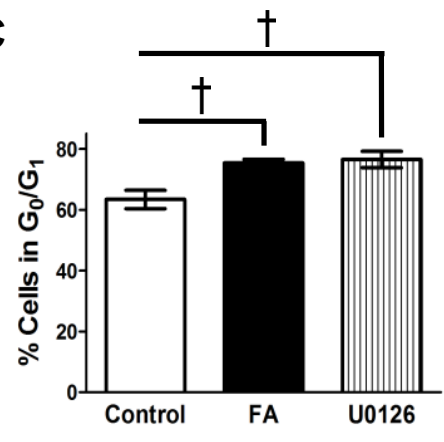

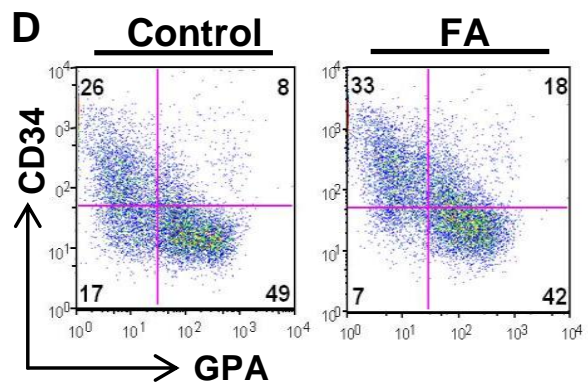

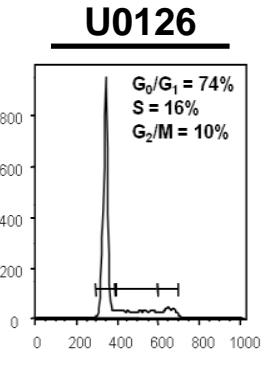

E
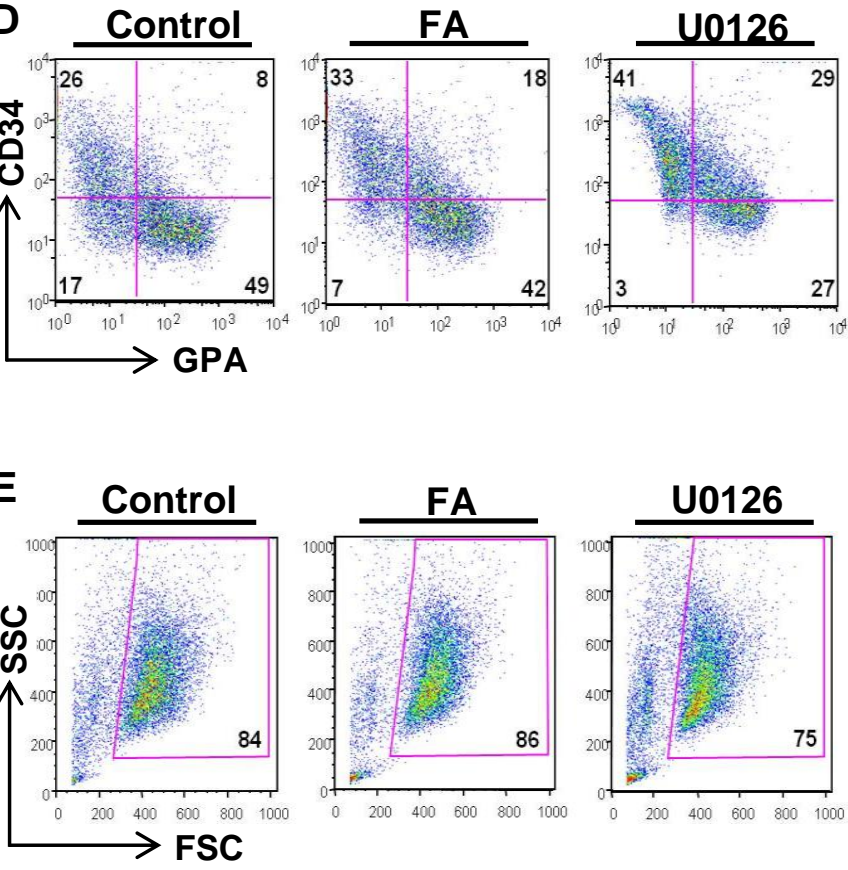

FC 
increased cells in $\mathrm{G}_{0} / \mathrm{G}_{1}$ (Figure 2.8B-C). MEK inhibition also impaired erythroid differentiation in a manner similar to aconitase inhibition, characterized by the accumulation of an aberrant $\mathrm{CD} 34^{+} \mathrm{GPA}^{+}$double positive population (Figure 2.8D). As with aconitase inhibition, MEK inhibition minimally affected cell viability (Figure 2.8E). The similar phenotypic effects resulting from FA and U0126 treatments thus support the notion that aconitase and ERK signaling participate in a common erythroid regulatory pathway.

\section{An aconitase-ERK protein complex that is disrupted by aconitase inhibition}

Large scale proteomic mapping of interaction networks in Saccharomyces cerevisiae has shown mitochondrial aconitase to interact with $~ 20-30$ partner proteins, of which one is KSS1, a yeast homolog of ERK; reciprocal analysis of KSS1 also identified aco1 (yeast mitochondrial aconitase) as an interaction partner [185]. Furthermore, high stringency scanning for motifs using Scansite 2.0 predicts ERK Docking Domains (DDomains) in both aconitase isoforms [186]. To test for interaction between aconitase enzymes and ERK in a mammalian system, immunoprecipitation was conducted on epitope-tagged proteins co-expressed in HEK293T cells cultured under a variety of conditions, including FA and EGF treatment. Higher FA doses were used in these experiments due to the relative resistance of HEK293T cells to the growth inhibitory effects of FA, most likely related to high levels of endogenous aconitases. ERK bound both aconitase isoforms, showing a stronger interaction with mitochondrial aconitase 
Figure 2.9 Identification of an ERK-aconitase protein complex that is disrupted by aconitase inhibition. (A) Assessment of ERK-aconitase interactions by coexpression followed by coimmunoprecipitation. ERK2 and FLAG-tagged cytosolic aconitase (cAcon-FLAG) or mitochondrial aconitase (mAcon-FLAG) were coexpressed in HEK293T cells $\pm 500 \mu \mathrm{M}$ FA. Immunoprecipitation (IP) with FLAG beads was followed by immunoblotting (IB) for total ERK-and for the FLAG epitope. (B) Role of ERK D-domain interacting motif in ERK2 binding to mitochondrial aconitase. mAconFLAG was coexpressed with ERK2 WT or D319N in HEK293T cells $\pm 500 \mu \mathrm{M}$ FA, \pm $100 \mathrm{ng} / \mathrm{ml}$ EGF, followed by IP and IB as in panel $\mathbf{A}$. 
Figure 2.9 Identification of an ERK-aconitase protein complex that is disrupted by aconitase inhibition.

A

\begin{tabular}{l} 
ERK2 WT \\
\hline cAcon- mAcon- \\
FLAG FLAG
\end{tabular}

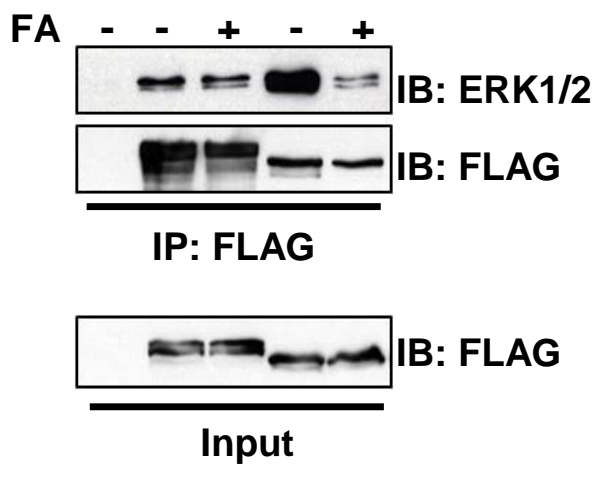

B

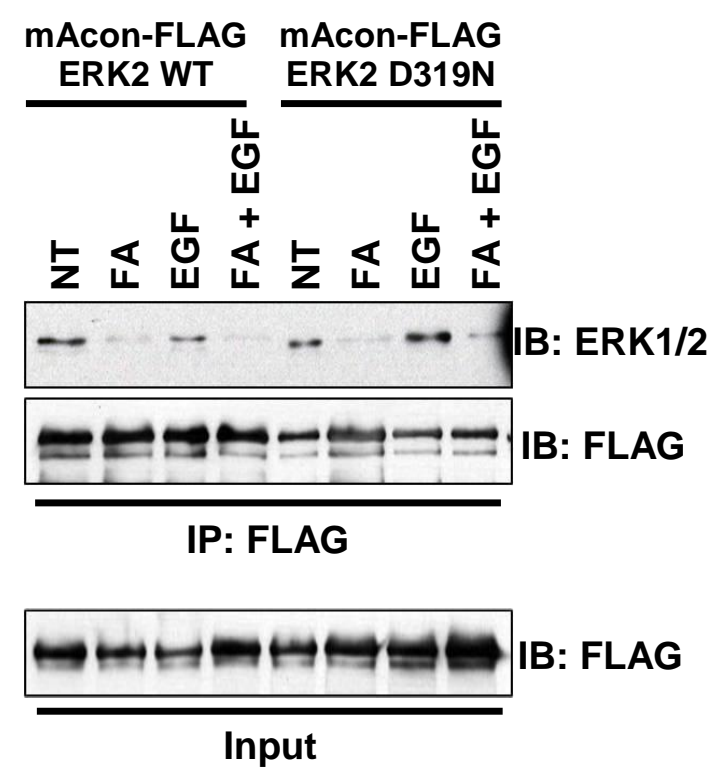


(Figure 2.9A). FA treatment potently and reproducibly diminished ERK2 interaction with mitochondrial aconitase. ERK activation by EGF treatment of serum-starved cells did not significantly affect the interaction, nor did mutation of the D-domain binding site in ERK2 (D319N) (Figure 2.9B) [187]. ERK1 was also found to interact with mitochondrial aconitase (data not shown). Our data thus confirm an interaction of mitochondrial aconitase with ERK1/2, reinforcing previous findings in Saccharomyces cerevisiae. This interaction is disrupted by inhibition of aconitase activity but does not appear to depend on ERK activation or D-domain recognition. Activation of cytoplasmic ERK targets such as RSK has been shown to depend on dimerization of ERK monomers [188]. Dimerization occurs in response to mitogenic stimuli, such as EGF in HEK293T cells, and provides a mechanism to spatially regulate ERK signaling. To test whether interaction with aconitase regulates ERK dimer formation, HEK293T cells \pm FA and EGF were subjected to ERK2 dimer detection according to the method of Casar et al [188]. FA treatment had no effect on ERK2 dimer formation (Figure 2.10), arguing against a role for aconitase in regulating ERK dimerization.

\section{Low dose aconitase inhibition normalizes red cell counts in a mouse model of polycythemia vera}

To determine whether aconitase inhibition could provide a therapeutic strategy for lowering red cell mass in polycythemia vera, we treated C57BL/6 mice expressing a 
Figure 2.10 Aconitase inhibition does not prevent ERK dimerization. Detection of ERK2 dimer formation upon EGF stimulation by native gel electrophoresis followed by immunoblotting. HEK293T cells $\pm 500 \mu \mathrm{M}$ FA, $\pm 10 \mu \mathrm{M}$ U0126 were cytokine-starved overnight and stimulated with $100 \mathrm{ng} / \mathrm{ml}$ EGF for 5 minutes prior to harvest. 
Figure 2.10 Aconitase inhibition does not prevent ERK dimerization.

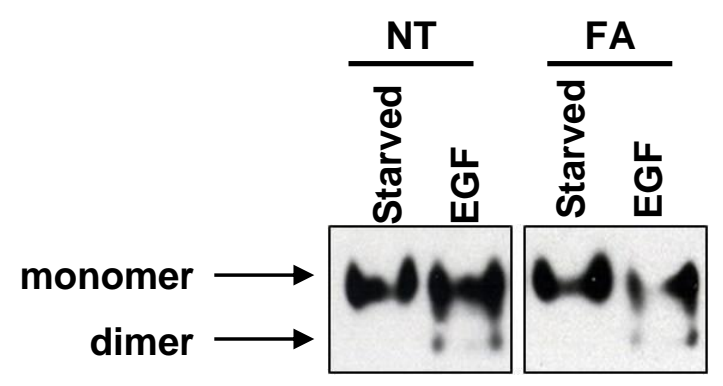


human mutant JAK2V617F transgene (PV mice) and wild type (WT) littermate controls with a low dose infusion of FA (2 mg/kg/day) [151]. Mock-treated PV mice had significantly elevated red cell counts compared with normal controls $\left(10.4-10.7 \times 10^{6} / \mu \mathrm{l}\right.$ in PV vs. 8.4-8.7 x 10\% $/ \mu$ in WT) (Figure 2.11A). FA infusion normalized the RBC counts in PV mice after seven days and stably maintained these counts in the normal range for the duration of treatment (Figure 2.11A). Platelet, neutrophil, and lymphocyte counts were not affected by FA treatment (Table 2.3). In PV marrows, as previously seen in WT marrows, FA increased the proportion of R3 erythroid precursors. A significant decrease in the percentage of more mature R4 precursors was also observed (Figure 2.11B-C). These results suggest that a minor degree of aconitase inhibition in vivo may suffice to achieve clinically significant reductions in red cell mass in polycythemia vera.

To determine effects of aconitase inhibition on signaling by the constitutively active mutant JAK2V617F, Ba/F3 transfectants expressing EPOR $\pm \mathrm{JAK} 2 \mathrm{~V} 617 \mathrm{~F}$ $[189,190]$ underwent treatment with FA. These cells however were resistant to growth inhibition by FA doses 20 -fold higher than those effective on primary erythroid progenitors (Figure 2.12A-B). As an additional complication, the Ba/F3 EPOR JAK2V617F cells showed a paradoxical response to EPO stimulation consisting of marked downregulation of RSK phosphorylation (Figure 2.12C). 
Figure 2.11 Aconitase inhibition normalizes red cell counts in a murine model of polycythemia vera. (A) RBC values over the course of a four-week infusion of saline $(0.9 \% ; \mathrm{n}=3)$ or FA (2 mg/kg/day; $\mathrm{n}=10)$. Data represent mean $\pm \mathrm{SD}$. (B) Erythroid maturation in day $28 \mathrm{PV}$ marrows. Boxes indicate erythroid developmental stages R1R4. (C) Summary of data from panel $\mathbf{B}$ with mean percentages of cells in $\mathrm{R} 1-\mathrm{R} 4 \pm \mathrm{SD}$; $\mathrm{n}$ $=3$ per group; ${ }^{*} P<0.05 ;{ }^{\dagger} P<0.01$. 
Figure 2.11 Aconitase inhibition normalizes red cell counts in a murine model of polycythemia vera.

A
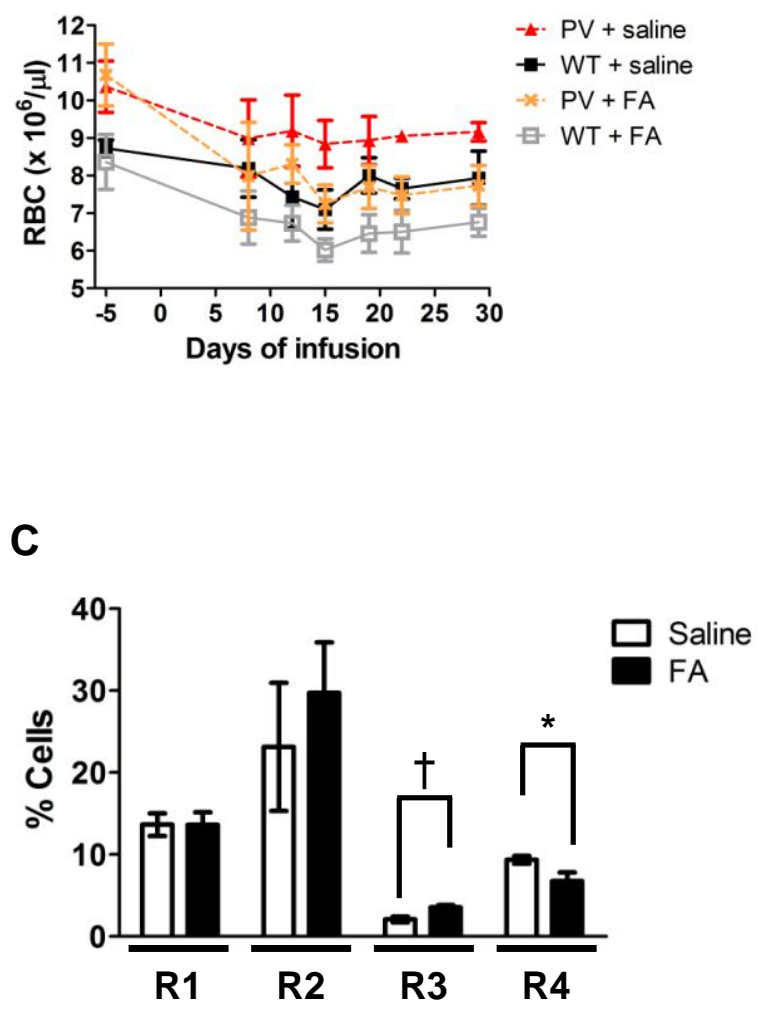

B

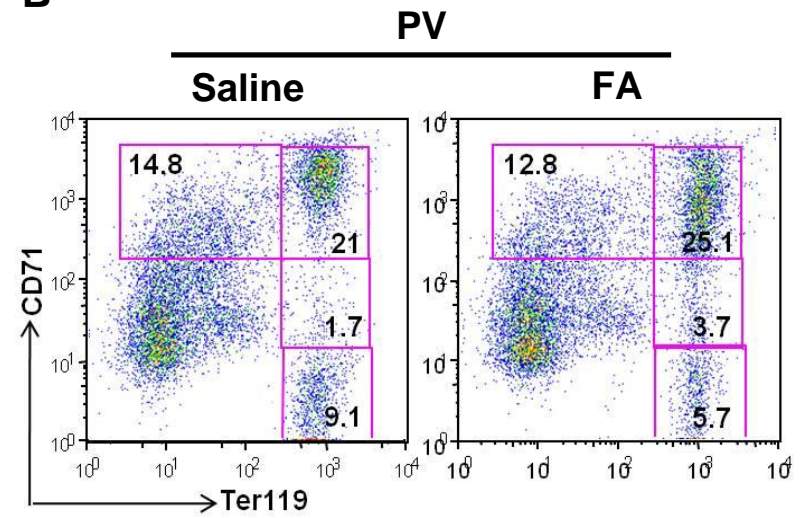




\section{Figure 2.12 Effects of FA and EPO on Ba/F3 cells expressing EPOR or EPOR}

JAK2V617F. (A) Proliferation of Ba/F3 EPOR cells cultured in the presence of $2 \mathrm{ng} / \mathrm{ml}$ murine IL-3 and treated with 0 - 5 mM FA for 72 hours. Data are presented as mean \pm $\mathrm{SD} ; \mathrm{n}=3 ; * P<0.05 ;$ n.s., not significant. (B) Proliferation of Ba/F3 EPOR

JAK2V617F cells cultured as in panel A. Data are presented as mean $\pm \mathrm{SD} ; \mathrm{n}=3 ;{ }^{\dagger} P<$ 0.01. (C) Paradoxical RSK deactivation in Ba/F3 EPOR JAK2V617F cells in response to EPO stimulation. Ba/F3 EPOR and Ba/F3 EPOR JAK2V617F cells were starved of cytokines for four hours prior to stimulation with $10 \mathrm{U} / \mathrm{ml}$ EPO for ten minutes.

Phosphorylation of ERK and RSK was assessed by immunoblotting of whole cell lysates. 
Figure 2.12 Effects of FA and EPO on Ba/F3 cells expressing EPOR or EPOR JAK2V617F.

A
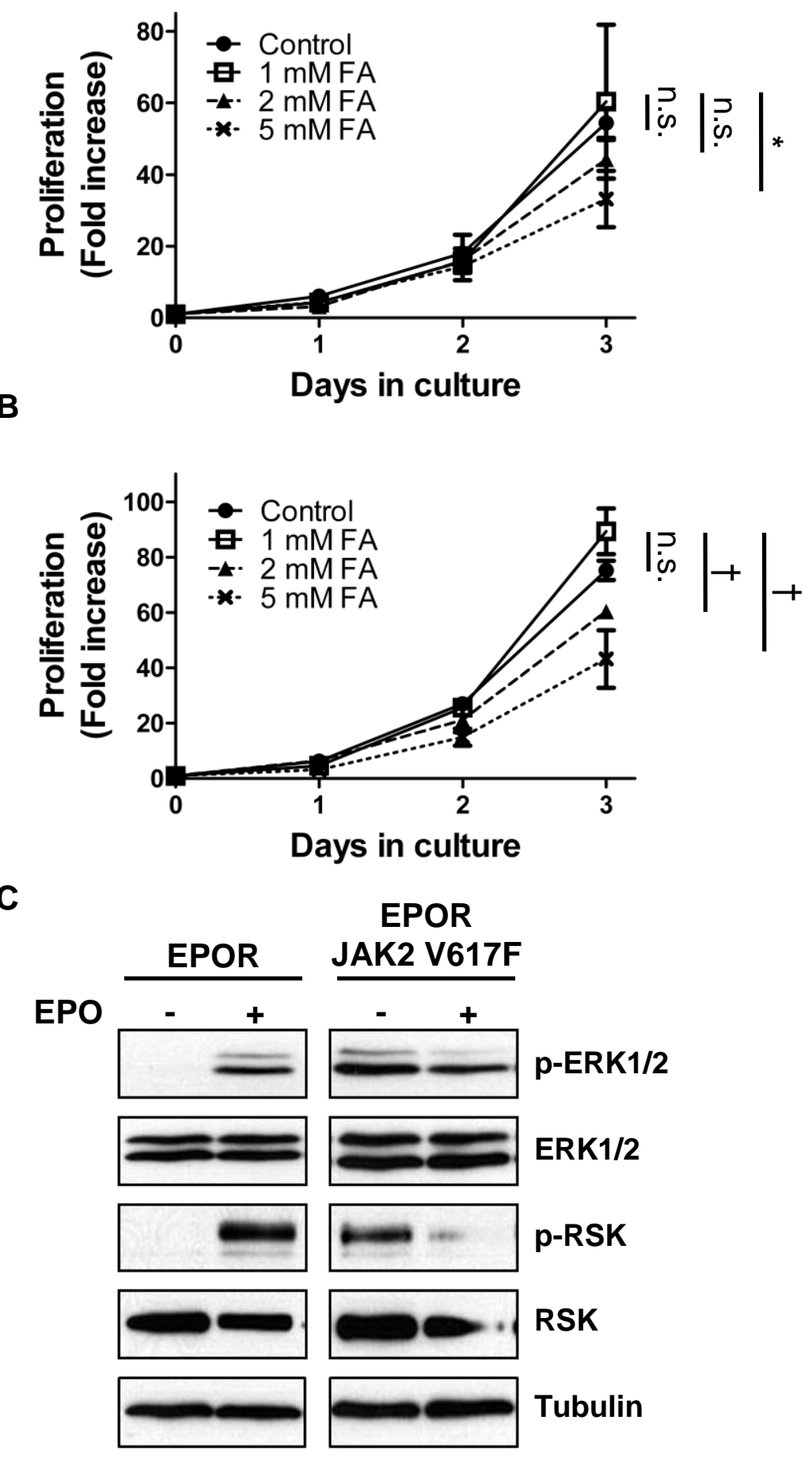
Table 2.2 CBC parameters of WT mice treated for four weeks with saline $(0.9 \%)$ or FA $(2 \mathrm{mg} / \mathrm{kg} / \mathrm{day})$.

\begin{tabular}{|c|c|c|c|}
\hline Blood parameter & $\begin{array}{l}\text { Saline } \\
(n=3)\end{array}$ & $\begin{array}{c}\text { FA-treated } \\
(n=10)\end{array}$ & $\begin{array}{c}P \\
\text { value } \\
\end{array}$ \\
\hline $\mathrm{RBC} \times 10^{6} / \mu \mathrm{L}$ & $7.93 \pm 0.72$ & $6.76 \pm 0.37$ & $0.0024^{*}$ \\
\hline Hematocrit, \% & $35.60 \pm 3.64$ & $34.62 \pm 1.39$ & 0.47 \\
\hline Hemoglobin, g/dL & $12.20 \pm 1.56$ & $11.76 \pm 1.45$ & 0.18 \\
\hline MCHC, g/dL & $34.57 \pm 4.77$ & $33.98 \pm 1.13$ & 0.70 \\
\hline MCV, fL & $44.83 \pm 0.6$ & $51.16 \pm 1.24$ & $<0.0001^{*}$ \\
\hline Platelets, $x 10^{3} / \mu \mathrm{L}$ & $866 \pm 46$ & $866 \pm 147.6$ & 0.99 \\
\hline Neutrophils, $\times 10^{3} / \mu \mathrm{L}$ & $1.78 \pm 1.00$ & $2.34 \pm 1.13$ & 0.43 \\
\hline Lymphocytes, $x 10^{3} / \mu \mathrm{L}$ & $5.50 \pm 2.04$ & $5.68 \pm 0.77$ & 0.91 \\
\hline
\end{tabular}

Table 2.3 CBC parameters of PV mice treated for four weeks with saline (0.9\%) or FA ( $2 \mathrm{mg} / \mathrm{kg} / \mathrm{day})$.

\begin{tabular}{|c|c|c|c|}
\hline Blood parameter & $\begin{array}{l}\text { Saline } \\
(\mathrm{n}=3)\end{array}$ & $\begin{array}{c}\text { FA-treated } \\
(n=10)\end{array}$ & $\begin{array}{c}P \\
\text { value }\end{array}$ \\
\hline $\mathrm{RBC}, \times 10^{6} / \mu \mathrm{L}$ & $9.16 \pm 0.25$ & $7.73 \pm 0.54$ & $.002^{*}$ \\
\hline Hematocrit, \% & $38.20 \pm 2.20$ & $35.41 \pm 1.83$ & $.037^{*}$ \\
\hline Hemoglobin, g/dL & $13.53 \pm 0.57$ & $12.59 \pm 0.72$ & .068 \\
\hline MCHC, g/dL & $35.47 \pm 0.67$ & $35.93 \pm 3.0$ & .8 \\
\hline MCV, fL & $40.2 \pm 1.04$ & $45.52 \pm 1.56$ & $.0003^{*}$ \\
\hline Platelets, $x 10^{3} / \mu \mathrm{L}$ & $1841 \pm 672$ & $1785 \pm 416$ & .86 \\
\hline Neutrophils, $x 10^{3} / \mu \mathrm{L}$ & $1.43 \pm 0.41$ & $3.91 \pm 2.42$ & .25 \\
\hline Lymphocytes, $x 10^{3} / \mu \mathrm{L}$ & $5.11 \pm 0.17$ & $6.97 \pm 3.17$ & .35 \\
\hline
\end{tabular}

Data are presented as mean \pm SD.

*indicate values that are significantly different from salinetreated mice. 


\section{Discussion}

Inactivation of aconitase enzymes contributes to the erythroid response to iron restriction [87]. However, because iron deprivation simultaneously affects multiple signaling and metabolic pathways, the exact role of aconitases in erythropoietic regulation has remained unclear. The current studies have directly examined the role of aconitases during erythroid development by use of a specific active site inhibitor. Application of this approach has confirmed that aconitase activity potently regulates erythropoiesis in vivo and in vitro. In vivo, aconitase inhibition caused a rapid-onset hypoplastic anemia with slightly macrocytic, normochromic red cells (Table 2.1). These red cell features suggest that the anemia did not arise from impaired production of heme due to deficiency of succinyl CoA. Anemias caused by defective heme synthesis, such as those due to deficiencies of erythroid-specific $\delta$-aminolevulinate synthase or ferrochelatase, are characteristically microcytic and hypochromic [191,192]. The low reticulocyte counts combined with increased serum EPO levels (Figure 2.2A-B) suggest that FA treatment interfered with red cell production, a conclusion further supported by the poor reticulocyte response to phenylhydrazine challenge seen in FA-treated animals (Figure 2.4B). Furthermore, in vivo and in vitro studies both showed impaired differentiation of erythroid progenitors associated with aconitase inhibition (Figures 2.2C, 2.5E, 2.11B).

Precise definition of the isoform-specific contributions of aconitases to erythroid regulation will require development of new conditional knockout mice with individual and compound gene deletions. Several lines of evidence, however, suggest that 
mitochondrial aconitase plays a key role. Firstly, fluoroacetate acts predominantly as an inhibitor of mitochondrial aconitase, based on the location of its enzymatic conversion to fluorocitrate, the active inhibitor [193]. Secondly, infusion of FA in mice lacking cytosolic aconitase (IRP1 -/-) induced an anemia identical to that occurring in wild-type mice (Figure 2.13). Thirdly, IRP1 -/- mice showed no hematologic defects in response to anemic challenge (Figure 2.4C-D). Fourthly, mitochondrial aconitase bound ERK more avidly than cytosolic aconitase and, unlike its cytosolic counterpart, displayed modulation of ERK binding by FA (Figure 2.9). Several studies have previously demonstrated mitochondrial regulation of erythroid differentiation and proliferation. For example, the inhibition of mitochondrial biogenesis by chloramphenicol is associated with impaired erythropoiesis in vivo and in vitro $[194,195]$. The ability of exogenous isocitrate to reverse in vitro differentiation defects caused by chloramphenicol has suggested that mitochondrial aconitase may participate in the regulation of erythropoiesis by mitochondrial function [87]. Further supporting this notion, multiple murine models of impaired mitochondrial function all show a similar, distinctive erythroid maturation defect, i.e. an increase in CD71 $1^{\text {Intermediate }}$ Ter $119^{\text {Bright }}$ progenitors (R3 stage), closely resembling the defect seen with FA infusion (Figures 2.2C and 2.11B) [196-198].

Among the various pathways downstream of EPO regulating erythroid development, only the ERK pathway displayed consistent modulation in response to aconitase inhibition. In particular, FA treatment diminished RSK T573 phosphorylation in erythroid progenitors subjected to either acute or chronic EPO stimulation (Figure 2.7). The effects of aconitase inhibition on ERK signaling are likely to be biologically relevant 
because direct inhibition of ERK activation with the MEK inhibitor U0126 recapitulated several key features of the erythroid response to FA (Figure 2.8). By contrast, our data suggest that the effects of FA were unrelated to changes in ATP, ROS, or mitochondrial membrane potential (Figure 2.6). Why FA had no effect on these latter parameters may be related to the dose employed, to possible metabolic compensation by cytosolic aconitase, or to the dependence of early hematopoietic progenitors more on glycolysis than on oxidative phosphorylation.

The mechanism underlying the cross-talk between aconitase and ERK, while unclear, may relate to the assembly of protein complexes containing ERK and mitochondrial aconitase (Figure 2.9). Interestingly, such complexes are phylogenetically conserved back to yeast and are regulated by aconitase activity. Previous studies have documented localization of ERK in mitochondria [172,199,200]. Whether mitochondrial aconitase participates in an organelle-specific ERK scaffolding complex, as has been described for endosomes and Golgi [169,170], remains a subject of investigation. Nevertheless, the current studies provide a novel function for aconitase as a licensing factor for erythropoietin-dependent transduction of ERK signaling. This regulatory input could permit adjustment of ERK activity in accordance with aconitase function, which in turn is affected by iron availability, redox, and iron-sulfur cluster assembly pathways. In this manner, a variety of signals reflecting mitochondrial status potentially could be transmitted to the machinery driving proliferation and differentiation. 
Figure 2.13 Mice lacking cytosolic aconitase respond normally to fluoroacetate infusion.

(A) RBC and hematocrit values in WT, IRP1+/- and IRP1-/- mice treated for 23 days with $(2 \mathrm{mg} / \mathrm{kg} /$ day $)$. Data represent mean $\pm \mathrm{SD} ; \mathrm{n}=6$ per group. (B) Absolute reticulocyte counts in the WT, IRP1-/WT and IRP1-/- groups, as in panel A. (C) Erythroid maturation in marrows from FA-treated animals. Representative flow cytometry plots are shown. Boxes indicate erythroid developmental stages R1-R4. (D) Summary of results from panel $\mathbf{C}$ showing mean percentages of cells in R1-R4 $\pm S D ; n=$ 3 per group. 
Figure 2.13 Mice lacking cytosolic aconitase respond normally to fluoroacetate infusion.

A
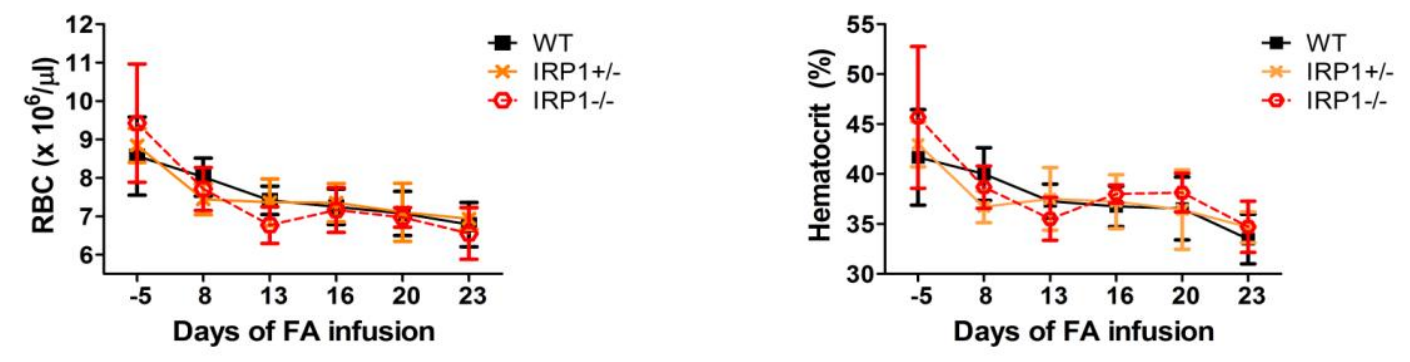

B

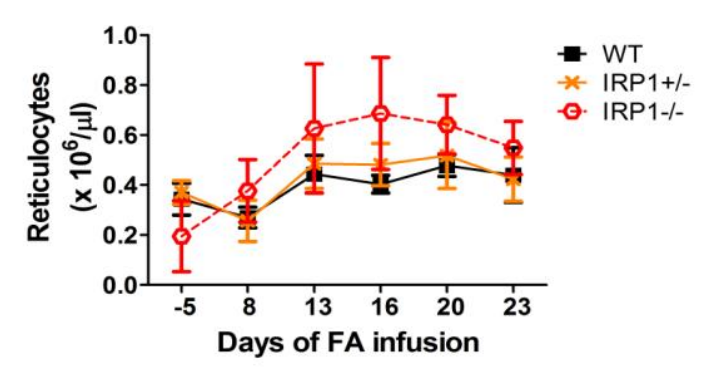

C
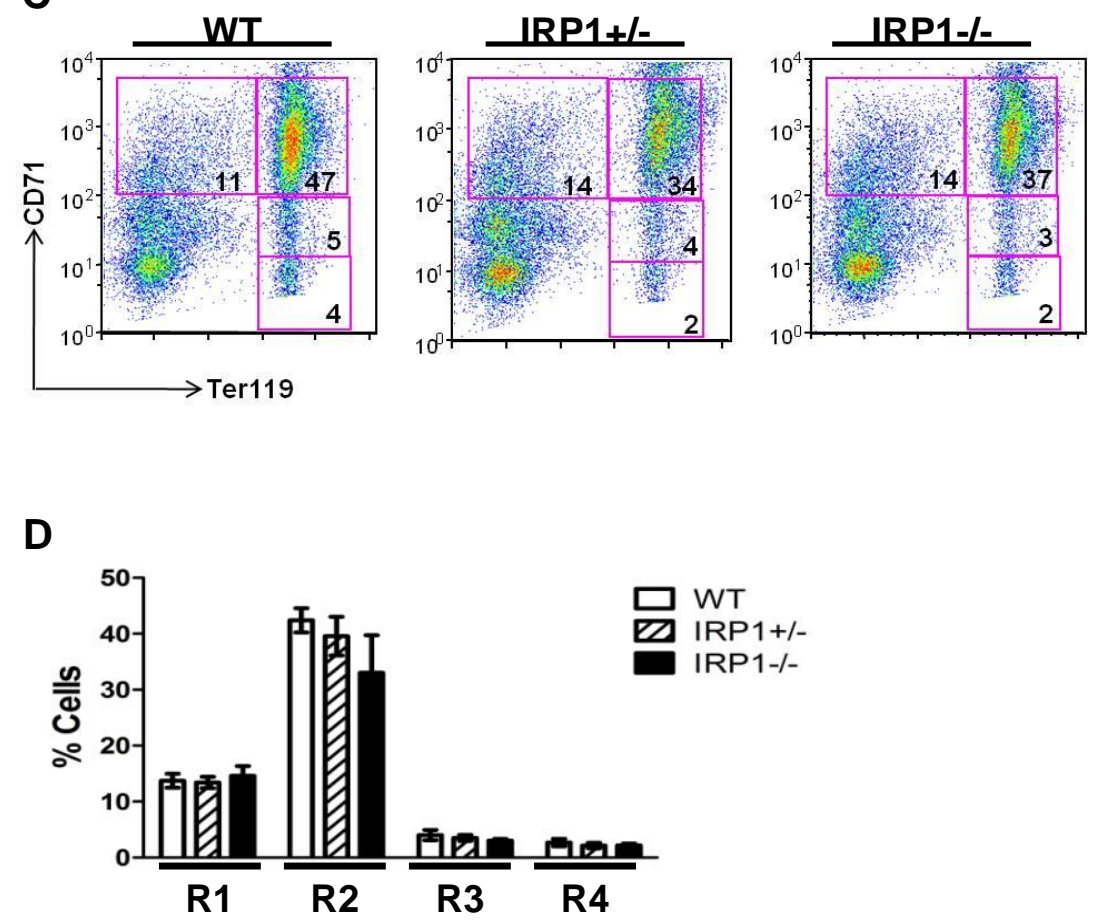
The ability to modulate red cell production with low dose aconitase inhibition may have therapeutic implications for polycythemia vera. Phlebotomy, the primary treatment for this disease, controls red cell mass via induction of iron deficiency, most likely acting through erythroid inactivation of aconitase. Attainment of iron deficiency may require numerous blood draws over a prolonged period and may be complicated by significant thrombocytosis [157]. Repeated phlebotomy also poses a risk for phlebitis and infection. In a mouse model of JAK2V617F-driven polycythemia, low dose aconitase inhibition rapidly normalized red cell counts in a lineage specific manner without causing anemia (Figure 2.11). However, further studies are needed to determine the clinical feasibility of this approach. Fluoroacetate is a known toxic agent that targets the cardiovascular and central nervous systems in mice and humans. Reported lethal doses range from 0.5 to $19 \mathrm{mg} / \mathrm{kg}$ in mice and 2 to $10 \mathrm{mg} / \mathrm{kg}$ in humans [120]. While a continuous FA infusion of $2 \mathrm{mg} / \mathrm{kg} /$ day corrects red cell counts in PV mice in the absence of overt symptoms, toxicology studies will be necessary to rule out adverse effects of both acute and chronic administration. 


\section{Materials and methods}

\section{Mice}

PV mice consisted of line A JAK2V617F transgenic mice between the ages of 10 and 14 weeks [151]. IRP1 -/- mice were generously donated by Dr. Tracey Rouault (National Institute of Child-Health \& Human Development, Bethesda, MD) [130]. Animal studies were approved by the University of Virginia Animal Care and Use Committee (ACUC, protocol 3545, latest annual renewal approved on 6.15.10). Mice were housed in a pathogen-free facility and handled in accordance with the policies of the University of Virginia ACUC. For continuous in vivo infusions, a sterile $0.9 \%$ saline solution or sodium fluoroacetate (FA) solution prepared in $0.9 \%$ saline was delivered subcutaneously using Alzet osmotic pumps model 1002 or 1004 (Durect, Cupertino, CA) based on the approach of Isken and colleagues [174]. Phenylhydrazine (Sigma, St. Louis, MO) prepared in sterile phosphate-buffered saline (PBS) was administered by intraperitoneal bolus injection at doses of $30 \mathrm{mg} / \mathrm{kg} /$ day x 2 days (FA-treated mice) or $60 \mathrm{mg} / \mathrm{kg} / \mathrm{day}$ x 2 days (saline-treated mice). Blood was collected from the retro-orbital plexus into EDTAcoated micro tubes (BD Biosciences, Franklin Lakes, NJ) using heparinized microcapillary tubes. Complete blood counts were measured on a Hemavet 850FS automated analyzer (Drew Scientific, Dallas, TX). Serum EPO levels were determined with the Quantikine Mouse EPO ELISA kit (R\&D Systems, Minneapolis, MN). 


\section{Cell culture}

Peripheral blood mobilized, purified human $\mathrm{CD} 34^{+}$cells were obtained from the Hematopoietic Cell Processing Core at the Fred Hutchinson Cancer Center (Seattle, WA) as previously described [87]. After 72 hours in multi-lineage serum-free medium, the cells were seeded in serum-free erythroid differentiation medium consisting of Iscove's modified Dulbecco medium (IMDM) supplemented with $4.5 \mathrm{U} / \mathrm{ml}$ EPO (Amgen, Thousand Oaks, CA), 25 ng/ml stem cell factor (SCF) (PeproTech, Rocky Hill, NJ), bovine serum albumin (BSA, Sigma), and 100\% Fe-saturated transferrin (Stem Cell Technologies, Vancouver, Canada) [87]. Sodium fluoroacetate was purchased from Sigma, and U0126 from Cell Signaling Technology (Beverly, MA). For cytokine starvation experiments, cells that had been cultured in erythroid medium for 72 hours were washed in PBS and re-suspended in IMDM supplemented with $0.1 \%$ BSA for 3 hours, followed by stimulation with $4.5 \mathrm{U} / \mathrm{ml}$ EPO. Murine Ba/F3 EPOR and Ba/F3 EPOR JAK2V617F cell lines have been previously described [201]. Both cells lines were maintained in Roswell Park Memorial Institute (RPMI) medium supplemented with $10 \%$ fetal bovine serum (Invitrogen, Carlsbad, CA) and $2 \mathrm{ng} / \mathrm{ml}$ murine IL-3 (PreproTech). For cytokine starvation experiments, cells were washed twice in RPMI and cultured for 4 hours in RPMI prior to stimulation with $10 \mathrm{U} / \mathrm{ml}$ EPO (Amgen). 


\section{Plasmids and transfections}

pcDNA3-HA-ERK2 wt and D319N, as well as pcDNA3-T7-ERK1, were purchased from Addgene (Cambridge, MA). Full-length cDNAs for human ACO1 (encoding cytosolic aconitase) and $\mathrm{ACO} 2$ (encoding mitochondrial aconitase) (Open Biosystems, Huntsville, AL) were subcloned into the $\mathrm{XhoI}$ and NotI sites of pCMV Tag4A (Stratagene, La Jolla, CA) to express mAcon-FLAG and cAcon-FLAG. A 3' SalI, rather than XhoI, site was introduced into the ACO2 insert. Transient transfections in HEK293T cells were carried out using the calcium phosphate method, as previously described [202]. Cells were serum-started for 6 hours, followed by stimulation with $100 \mathrm{ng} / \mathrm{ml}$ epidermal growth factor (EGF) (PeproTech) for 5 minutes prior to harvest where indicated. 36 hours posttransfection, cells were resuspended in NP-40 extraction buffer containing $50 \mathrm{mM}$ Tris$\mathrm{HCl}$ (pH 7.6), $150 \mathrm{mM} \mathrm{NaCl}, 0.5 \% \mathrm{NP}-40,1 \mathrm{mM} \mathrm{MgCl} 2,50 \mu \mathrm{M} \mathrm{ZnSO}_{4}$, EDTA-free protease inhibitor cocktail (Roche, Indianapolis, IN), $1 \mathrm{mM}$ sodium orthovanadate and $10 \mathrm{mM}$ sodium fluoride. Following a 20 minute incubation at $4^{\circ} \mathrm{C}$, insoluble debris was removed by centrifugation.

\section{Immunoblot and immunoprecipitation}

Western blot analysis was performed as previously described after sodium dodecyl sulfate-poly-acrylamide gel electrophoresis (SDS-PAGE) [203]. Antibodies to phosphoERK1/2 (T202/Y204), ERK1/2, RSK1, phospho-STAT5 (Y694), STAT5, phospho-Akt 
(S473), Akt, AMPK, and phospho-AMPK (T172) were purchased from Cell Signaling Technology. Anti-phospho-RSK (T573) was purchased from Epitomics (Burlingame, CA); anti-caspase 3 from Santa Cruz Biotechnology (Santa Cruz, CA). Anti-tubulin and anti-actin were purchased from Sigma. Immunoprecipitation of aconitase-containing complexes was performed by incubating cellular extracts with M2 agarose FLAG beads (Sigma) for 2 hours at $4^{\circ} \mathrm{C}$. Following washing of beads, proteins eluted in SDS-PAGE loading buffer underwent SDS-PAGE and immunoblot analysis using antibodies to ERK1/2 (Cell Signaling Technology) and FLAG (Sigma). Densitometry data were acquired on a GS800 calibrated densitometer (Bio-Rad, Hercules, CA) and analyzed with the Quantity One software (Bio-Rad). For ERK2 dimer formation analysis, HEK293T cells were serum-starved overnight $\pm 500 \mu \mathrm{M}$ FA and stimulated with $100 \mathrm{ng} / \mathrm{ml}$ EGF for 5 minutes prior to harvest and analysis by native gel electrophoresis as described [204].

\section{Flow cytometry}

Cells were analyzed on a FACSCalibur instrument (Becton Dickinson, San Jose, CA). All fluorochrome-conjugated antibodies were purchased from BD Pharmingen (San Diego, CA), with the exception of PE-anti-CD71 (Dako, Carpinteria, CA). Erythroid differentiation was assessed by co-staining human erythroid progenitors with fluorochrome-conjugated antibodies to GPA (FITC or PE), CD71 (PE), CD34 (APC), and CD44 (FITC). Reticulocytes were measured by staining whole blood with thiazole orange as described [205]. Bone marrows were harvested from femurs into PBS 
supplemented with $5 \mathrm{mM}$ EDTA and stained with APC-anti-Ter119 and PE-anti-CD71. Cell death was analyzed with the Annexin V-PE Apoptosis Detection Kit I (BD Pharmingen). For cell cycle analysis, cells underwent ethanol fixation and RNase A treatment followed by propidium iodide staining. Data was analyzed using the FlowJo software (version 8.6.3, TreeStar Inc., Ashland, OR).

\section{Metabolic assays}

Citrate measurements were performed using the BioVision Citrate Assay kit according to the manufacturer's instructions (BioVision, Mountain View, CA). To measure intracellular levels, metabolic extracts from day 5 untreated and $50 \mu \mathrm{M}$ FA-treated $\mathrm{CD} 4^{+}$erythroid cultures were obtained as previously described [87]. Fluorimetry was performed on a FluoSTAR Optima fluorescent microplate reader (BMG Labtech, Cary, NC) and analyzed with the MARS data analysis software (version 1.01, BMG Labtech). To measure serum citrate, whole blood was collected retro-orbitally and allowed to clot at room temperature for 30 minutes. Clotted samples were centrifuged at $13,000 \mathrm{~g}$ for 5 minutes at room temperature and sera were stored at $-20^{\circ}$ C. Samples were deproteinized using the Deproteinization Sample Preparation kit (BioVision) according to the manufacturer's instructions.

ATP measurements were performed as described [87]. Staining of cells with 3'(aminophenyl)-fluorescein (APF) followed the manufacturer's guidelines (Invitrogen). Briefly, CD34 cells $\pm 50 \mu \mathrm{M}$ FA were stained with APC-anti-GPA (BD Pharmingen) and 
APF, and mean fluorescence intensity of the APF dye was determined by flow cytometry after gating on GPA-negative and positive populations. Mitochondrial membrane potential was determined by staining cells with MitoTracker Red CMXRos dye (Invitrogen) according to the manufacturer's instructions, followed by staining for GPA. The mean fluorescence intensity of MitoTracker stained cells was determined by flow cytometry after gating on GPA-negative and positive populations.

\section{Statistical analysis}

GraphPad Prism software version 5.01 (GraphPad Software, Inc., La Jolla, CA) was used to display the data graphically and to perform statistical analysis. Results were analyzed by unpaired two-tailed Student's $t$ test or one-way analysis of variance (ANOVA) with Newman-Keuls post-test when comparing multiple groups. $P$ values less than or equal to 0.05 were considered significant. 
CHAPTER 3:

Related Data 


\section{Are RSKs direct mediators of the effects of aconitase on erythropoiesis?}

\section{Pharmacologic inhibition of the RSK family of kinases}

Our signaling data indicates that ERK signaling is a mediator of the response to aconitase blockade in primary erythroid cultures. Specifically, a defect in ERK-mediated activation of RSK was consistently observed upon acute EPO stimulation (Figure 2.7). The RSK family of kinases is comprised of 4 highly homologous kinases, RSK1-4, and two structurally related kinases, MSK1-2 [184]. While MSKs can be activated by both ERK1/2 and p38 kinases, RSKs are exclusively activated by ERK1/2 [206,207]. RSK activation occurs in sequential steps; the first step consists of ERK-mediated phosphorylation of a threonine residue (threonine 573 in human RSK1) in the activation loop of the C-terminal kinase domain [208]. This activation event then directs further phosphorylation and full activation of the kinase. The failure to phosphorylate RSK on this critical threonine residue under aconitase inhibition suggested that RSK may be a key mediator of proliferation and differentiation signals in developing erythroid cells.

To assess a functional role for RSK during erythropoiesis, pharmacological inhibitors were employed. SL0101 is a novel, ATP-competitive, inhibitor of RSK with an in vitro $\mathrm{IC}_{50}$ of $89 \mathrm{nM}$ in the presence of $10 \mu \mathrm{M}$ ATP [209]. CD34 ${ }^{+}$cells were cultured in erythroid medium for five days in the presence of $50 \mu \mathrm{M}$ SL0101 or $50 \mu \mathrm{M}$ FA and the impact of RSK inhibition on differentiation, viability, and proliferation 
Figure 3.1 SL0101-mediated RSK inhibition recapitulates the effects of FA on erythroid maturation and proliferation. (A) Maturation profiles of day 5 erythroid cultures treated with $50 \mu \mathrm{M}$ FA or $50 \mu \mathrm{M}$ SL0101. (B) Mean percentage of GPA ${ }^{+} \mathrm{CD} 34^{-}$ cells from three independent experiments as in panel $\mathbf{A},{ }^{*} P<0.05 ;{ }^{\dagger} P<0.01$; n.s., not significant. (C) Viability of day 5 erythroid cultures treated with $50 \mu \mathrm{M}$ FA or $50 \mu \mathrm{M}$ SL0101. (D) Cell counts from three independent experiments set up as in panel A, ${ }^{\dagger} P<$ $0.01 ;{ }^{\ddagger} P<0.001 ;$ n.s., not significant. 
Figure 3.1 SL0101-mediated RSK inhibition recapitulates the effects of FA on erythroid maturation and proliferation. .

A
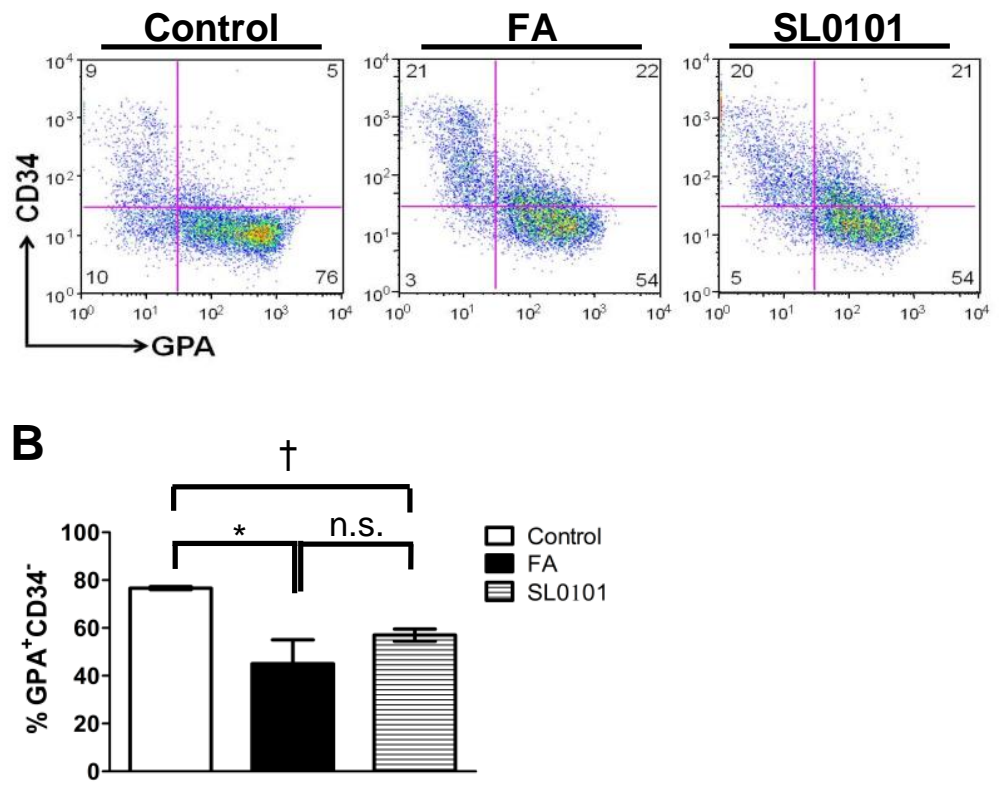

C

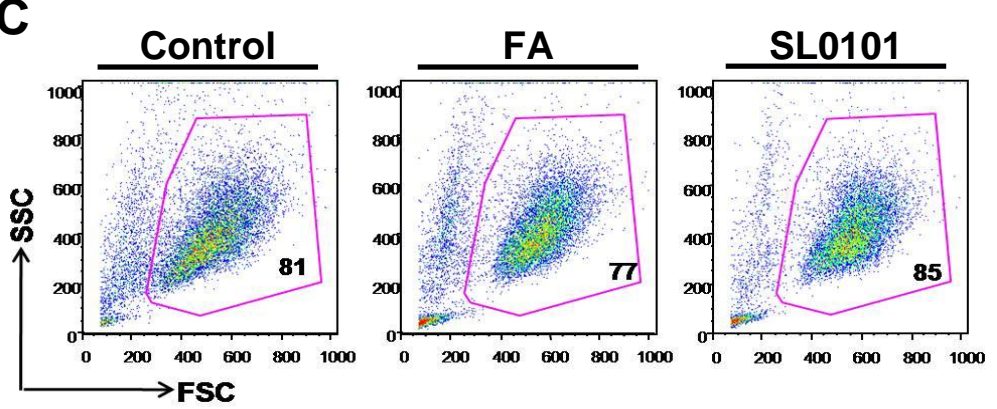

D

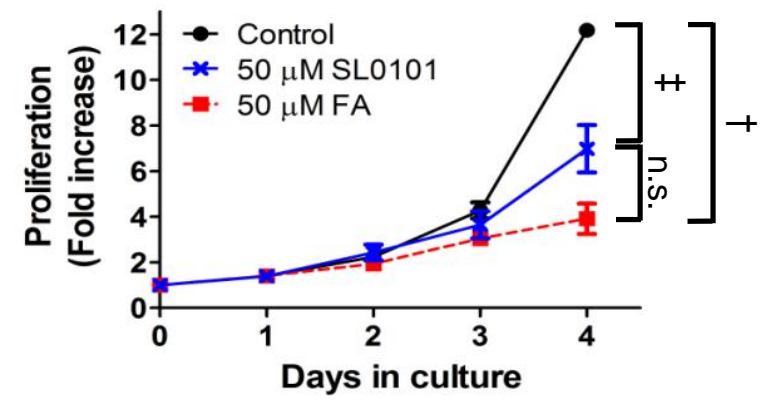


was compared to that of aconitase inhibition. The RSK inhibitor reproducibly impaired differentiation of erythroid progenitors, characterized by a decreased percentage of $\mathrm{CD}^{-}{ }^{-} \mathrm{GPA}^{+}$cells (Figure 3.1A-B). The degree of impairment was similar to that caused by FA treatment. Importantly, a $50 \mu \mathrm{M}$ dose of SL0101 did not affect viability (Figure 3.1C). Furthermore, RSK inhibition significantly reduced proliferation of erythroid progenitors (Figure 3.1D).

To validate these results, we turned to a more recently described, unrelated ATPcompetitive inhibitor of RSK, Bi-D1870. This compound was reported to have an $\mathrm{IC}_{50}$ of $5 \mathrm{nM}$ and $10 \mathrm{nM}$ against RSK1 and RSK2, respectively, in the presence of $10 \mu \mathrm{M}$ ATP [210]. Initial titration experiments conducted to find a suitable dose of Bi-D1870 in $\mathrm{CD} 34^{+}$cultures indicated that the compound had a very narrow window of tolerance, with 0.1-3 $\mu \mathrm{M}$ having no noticeable effects and $10 \mu \mathrm{M}$ causing severe cell death (not shown). The effects of $5 \mu \mathrm{M}$ Bi-D1870, a well-tolerated dose (Figure 3.2B) were compared with those of $50 \mu \mathrm{M}$ FA in erythroid cultures. In contrast with SL0101, BiD1870-mediated RSK inhibition had no impact on erythroid differentiation (Figure 3.2A). Furthermore, $5 \mu \mathrm{M}$ Bi-D1870 had a very minimal impact on erythroid proliferation over the course of five days (Figure 3.2C). Thus Bi-D1870 did not recapitulate the effects of SL0101 and the role of RSK in erythropoiesis remained unclear. 
Figure 3.2 Bi-D1870-mediated inhibition of RSK does not impair erythroid differentiation or proliferation. (A) Maturation profiles of day 5 erythroid cultures treated with $5 \mu \mathrm{M}$ Bi-D1870 or $50 \mu \mathrm{M}$ FA. (B) Viability of day 5 erythroid cultures treated with $5 \mu \mathrm{M}$ Bi-D1870 or $50 \mu \mathrm{M}$ FA. (C) CD34 $4^{+}$cells were cultured as in panel A and daily cell counts were enumerated. 
Figure 3.2 Bi-D1870-mediated inhibition of RSK does not impair erythroid differentiation or proliferation.

A

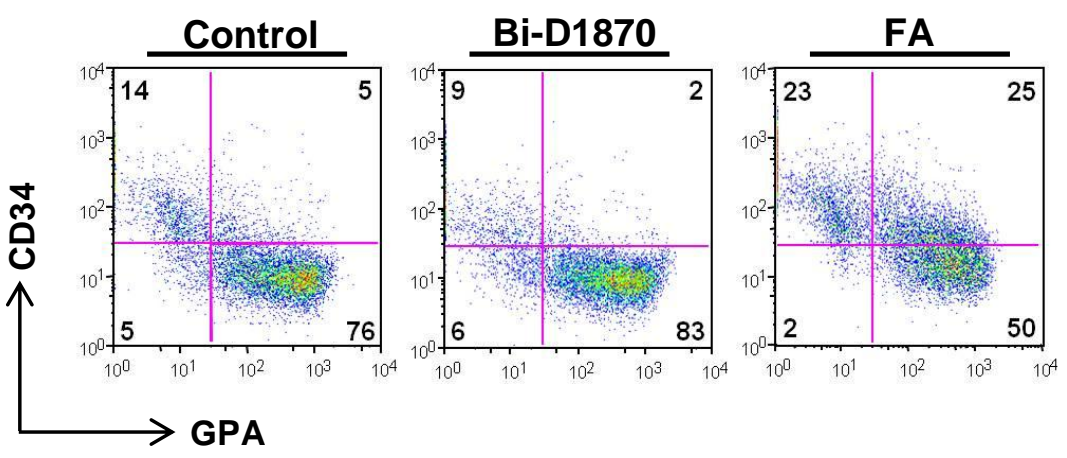

B
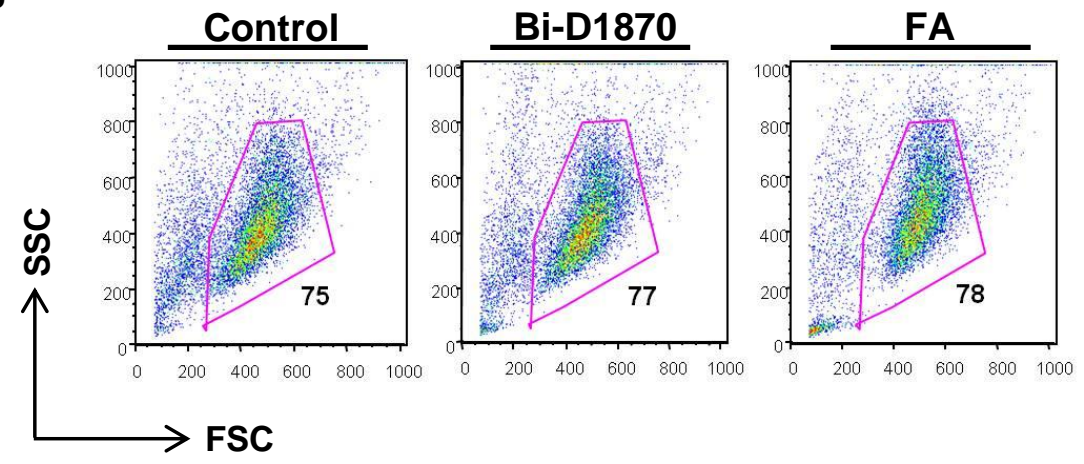

C

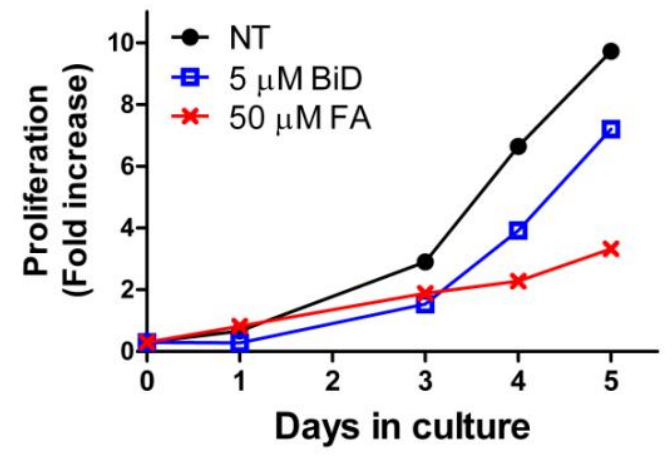


Impact of RSK mutants on erythroid differentiation

To address the discordant results obtained with pharmacologic inhibition of RSK, overexpression experiments were carried to assess the role of RSK activity on erythroid differentiation. $\mathrm{CD}^{+} 4^{+}$cells were nucleofected with expression plasmid for wild type RSK2 (RSK2 WT); a constitutively active mutant, RSK2 Y707A; or a dominant negative mutant, RSK2 K100A [211,212]. Differentiation was assessed by flow cytometry 72 hours and 96 hours post transfection. As shown in Figure 3.3, overexpression of mutant RSK2 did not affect erythroid differentiation. However, it is possible that other homologues, such as RSK1, were able to compensate for absent RSK2.

Transient knockdown of RSK1 or 2 does not diminish erythroid maturation

A significant challenge in studying the potential role(s) for RSK during erythropoiesis is due to the fact that there are 4 highly related homologues, RSK1-4 [184]. Mining of gene expression profile datasets derived from erythroid primary cells in the Gene Expression Omnibus repository revealed that RSK1 and RSK2 are the dominant isoforms expressed in erythroid progenitors. The functional impact of knocking down either RSK isoform was assessed in $\mathrm{CD}_{3} 4^{+}$cultures using small interfering RNAs (siRNA), following the method of Keerthivasan and colleagues [213]. Up to $100 \mathrm{nM}$ total siRNA dose transfected on two consecutive days was well tolerated by the primary cells. Despite a 50\% knockdown in RSK1 and RSK2 protein levels (Figure 3.4), erythroid differentiation was unaffected by the knockdowns. In the first experiment, 
Figure 3.3 Enforced expression of RSK kinase domain mutants does not affect erythroid differentiation of primary progenitors. (A) Erythroid differentiation of $\mathrm{CD} 4^{+}$cells 72 hours after nucleofection with wild type (WT), kinase dead (K100A), or constitutively active (Y707A) RSK2 constructs. Numbers indicate the percentage of cells in each quadrant. (B) Summary of three independent experiments conducted as in panel A. Data is presented as mean \pm SD. (C) Representative flow cytometry plots showing viability and nucleofection efficiency based on GFP expression in RSK2 WT transfected cells. Numbers indicate the percentage of cells in the gated region. Nucleofection of RSK K100A and Y707A mutants did not affect viability or transfection efficiency (not shown). 
Figure 3.3 Enforced expression of RSK kinase domain mutants does not affect erythroid differentiation of primary progenitors.

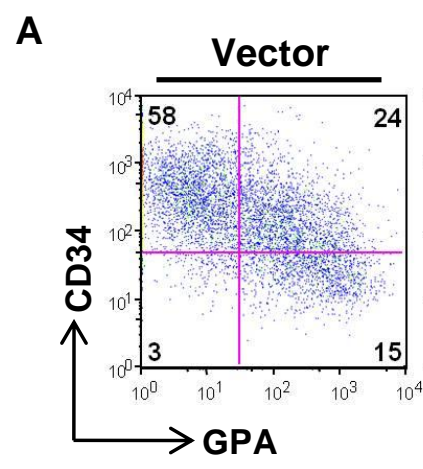

B

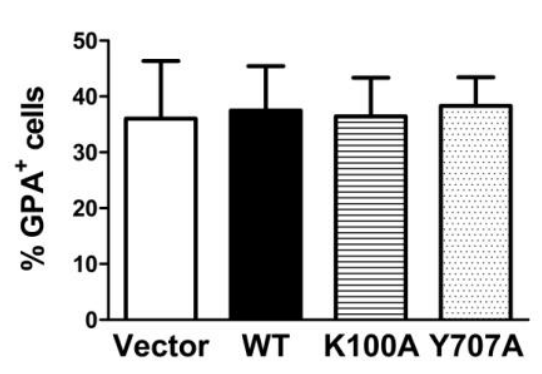

C

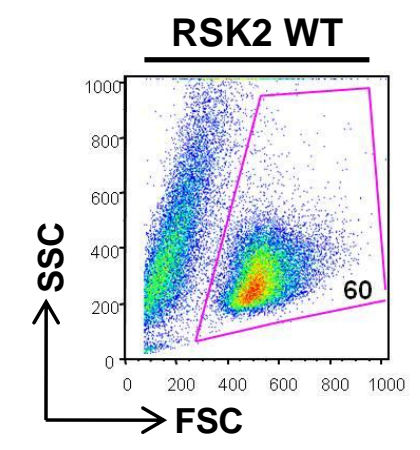


combined knockdown of RSK1 and RSK2 very modestly decreased the percentage of more mature $\mathrm{CD}^{-} 4^{-} \mathrm{GPA}^{+}$erythroid precursors (Figure 3.4). However, subsequent experiments looking at the effect of knocking down either RSK1 or RSK2 yielded opposite results (Figure 3.4). While it is possible that a more efficient knockdown of both isoforms, i.e., greater than 50\%, could yield more definitive data, RSK do not appear to mediate the effects of aconitase inhibition on erythroid maturation. While interesting, the decrease in phosphorylated RSK upon FA treatment may simply be a marker of deregulated ERK1/2 signaling. 


\section{Figure 3.4 Transient knockdown of RSK1 and RSK2 does not impair erythroid}

differentiation. (A) Differentiation of erythroid progenitor cells transiently transfected with $50 \mathrm{nM}$ siRNA against RSK1, RSK2 or both. Differentiation was assessed 72 hours post-transfection by flow cytometry. (B) Viability of erythroid progenitors transfected as in panel $\mathbf{A}$ after 72 hours. (C) Effects of a non-targeting siRNA negative control on differentiation and viability after 72 hours. (D) Whole cell lysates prepared at the 72 hour timepoint where analyzed by immunoblot for RSK1 and RSK2 protein levels. Tubulin is shown as a loading control. Lane 1: untransfected cells; lane 2: mocktransfected cells; lane 3: non-targeting siRNA; lane 4: siRNA againts RSK1 (50 nM) and RSK2 (50 nM). 
Figure 3.4 Transient knockdown of RSKs 1 and 2 does not impair erythroid differentiation.

A
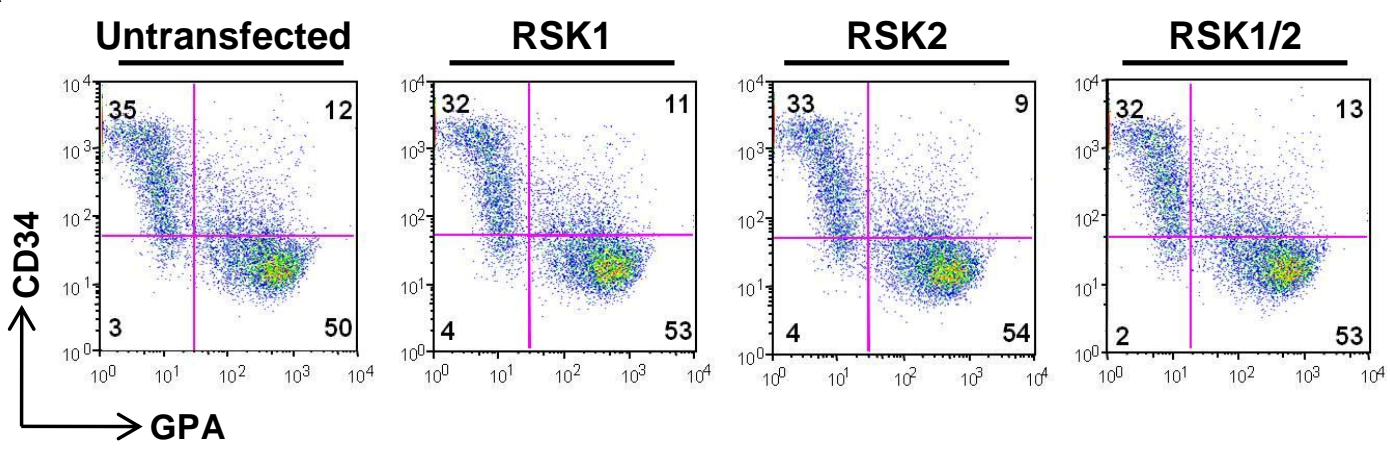

B
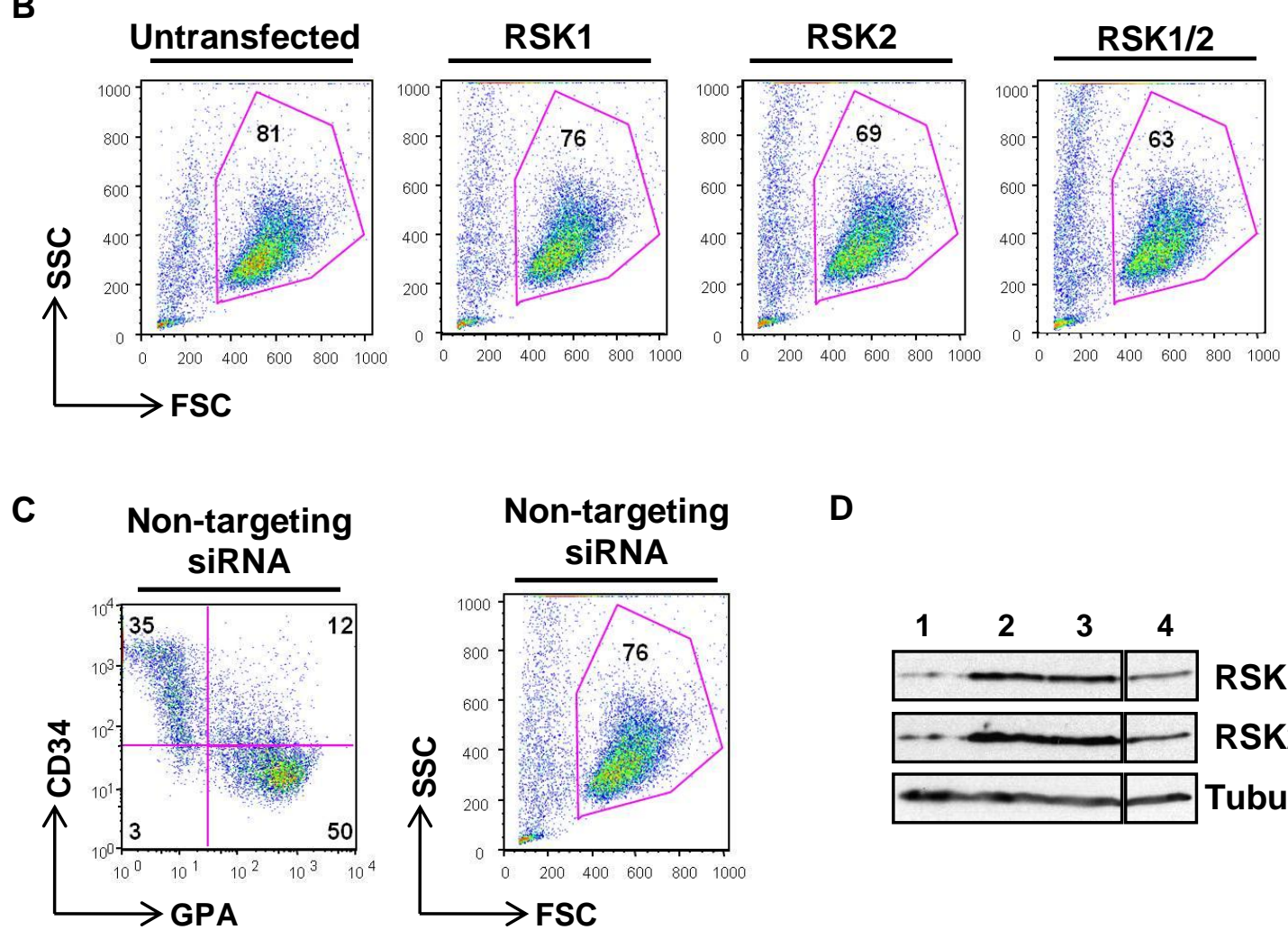

D

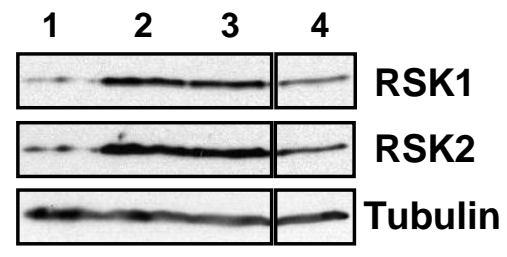




\section{Aconitase blockade impairs ERK-mediated activation of MNK}

The defect in ERK-mediated RSK activation in FA-treated erythroid progenitors raised the question of whether other cytosolic ERK targets may also be impaired in their activation. MAPK-interacting kinases (MNK) are ERK targets known to regulate the translational machinery, including eukaryotic initiation factor $4 \mathrm{~F}$, and mRNA binding proteins $[214,215]$. In FA-treated erythroid progenitors, ERK-dependent phosphorylation of MNK was decreased when compared to untreated cells, while total MNK1 protein levels were unchanged (Figure 3.5). These data provide additional support that aconitase inhibition may specifically disrupt ERK signaling to cytosolic targets. An inhibitor of MNK is now available and could be used in erythroid progenitor cultures to assess whether MNK is an important mediator of growth and differentiation during erythropoiesis [216]. 
Figure 3.5 Aconitase inhibition prevents ERK-directed phosphorylation of MNK. MNK and ERK1/2 phosphorylation in response to brief EPO stimulation. Cells from day 4 erythroid cultures $\pm 50 \mu \mathrm{M}$ FA and $\pm 10 \mu \mathrm{M}$ U0126 were subjected to 3 hours of cytokine starvation followed by stimulation with $4.5 \mathrm{U} / \mathrm{ml}$ EPO for 10 minutes. Tubulin is shown as a loading control. 
Figure 3.5 Aconitase inhibition prevents ERK-directed phosphorylation of MNK.

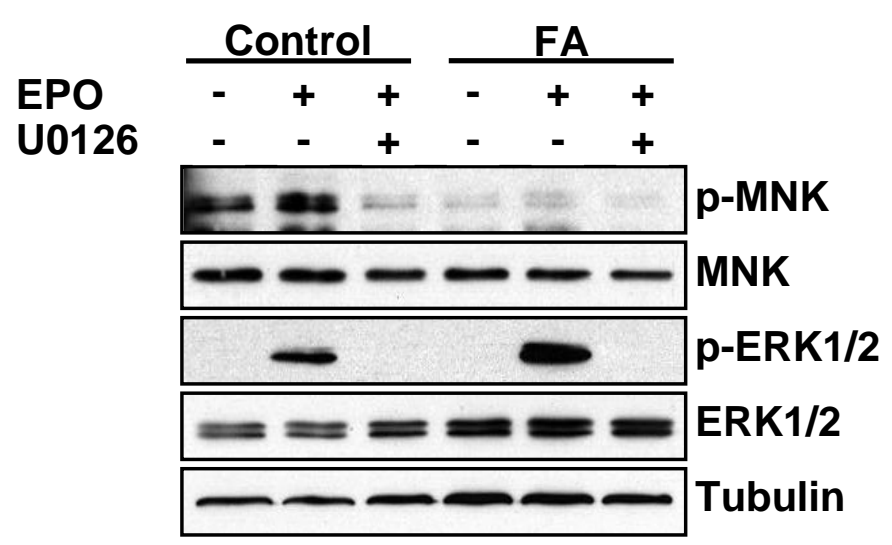




\section{MEK activation is not dynamically modulated by EPO}

Classically, Raf-MEK-ERK activation is dependent on growth factor stimulation. Under cytokine starvation conditions, phosphorylated levels of Raf, MEK1/2, and ERK1/2 are minimal and often below threshold of detection by immunoblotting. Growth factor binding to its cognate receptor causes a transient activation of the signaling cascade, which can be assessed by measuring levels of phosphorylated and total protein. Primary human erythroid progenitors have detectable baseline levels of phosphorylation of MEK1/2, despite a 3 hour starvation period in serum-free medium (Figure 3.5, lanes 1 and 7). Surprisingly, stimulation of starved progenitors with EPO did not cause any significant increase in p-MEK1/2 levels, while p-ERK1/2 levels rose considerably (Figure 3.6, lanes 1 vs. 2-5; lanes 7 vs. 8-10). This stands in contrast with ERK activation. Phospho-ERK1/2 levels were undetectable at baseline and increased transiently with EPO stimulation (Figure 3.6). This was a reproducible finding, observed in four independent experiments. These results suggest that erythroid progenitors maintain a steady level of activated MEK1/2 and that dynamic regulation of the MAPK cascade may occur at the level of ERK1/2. 
Figure 3.6 MEK activation is not dynamic in EPO stimulated erythroid progenitors. Time course of MEK1/2 and ERK1/2 phosphorylation. Cells from day 3 erythroid cultures $\pm 50 \mu \mathrm{M}$ FA were subjected to 3 hours of cytokine starvation followed by stimulation with $4.5 \mathrm{U} / \mathrm{ml}$ EPO for the indicated time periods. Tubulin is shown as a loading control. 
Figure 3.6 MEK activation is not dynamic in EPO stimulated erythroid progenitors.

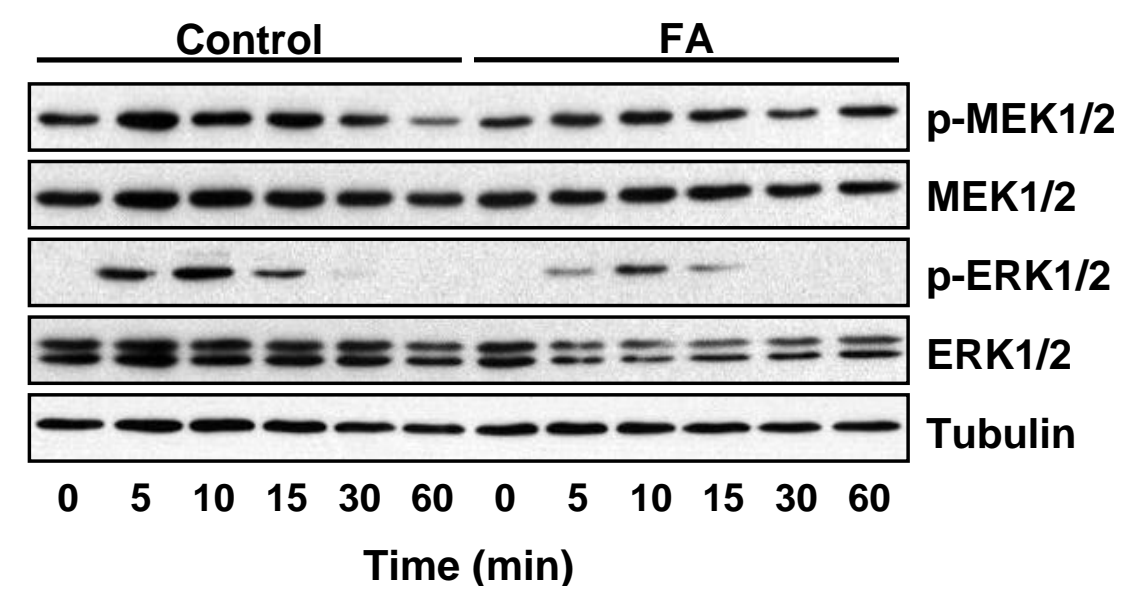




\section{Elk-1 activation is not dynamically modulated by EPO}

Elk-1 is an Ets-domain containing transcription factor classically activated by ERK1/2. Growth factor stimulation at the cell surface and signaling via the MAPK cascade results in nuclear translocation of activated ERK1/2 and phosphorylation of Elk-

1 [217]. In conjunction with other transcription factors, Elk-1 regulates the expression of many genes involved in proliferation, including $c-f o s$, a key regulator of proliferation and differentiation [218]. Since aconitase blockade correlated with impaired ERK-mediated RSK phosphorylation, the activation of Elk-1 was investigated. As shown in Figure 3.7, phosphorylated Elk-1 was present in cytokine-starved erythroid progenitors. Addition of EPO did not cause immediate upregulation of p-Elk-1 levels, as published data suggested. These observations suggest that EPO-induced MAPK signaling is not a regulator of Elk-1 activation. The presence of the aconitase inhibitor did not affect baseline or EPOstimulated levels of p-Elk-1 in these cultures (Figure 3.7). 
Figure 3.7 Elk-1 activation is not dynamic in EPO-stimulated erythroid progenitors. Time course of Elk-1 and ERK1/2 phosphorylation. Cells from day 3 erythroid cultures $\pm 50 \mu \mathrm{M}$ FA were subjected to 3 hours of cytokine starvation followed by stimulation with $4.5 \mathrm{U} / \mathrm{ml}$ EPO for the indicated time periods. Tubulin is shown as a loading control. 
Figure 3.7 Elk-1 activation is not dynamic in EPO-stimulated erythroid progenitors.

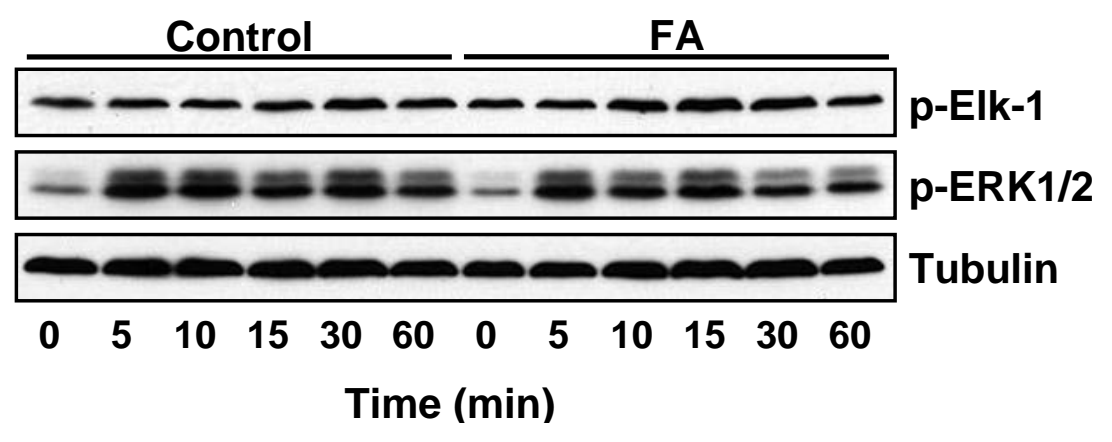




\section{Deregulated ERK signaling is not caused by defects in dephosphorylation}

ERK signaling is regulated in part by duration of stimulus as well as by inactivation of the kinase via dephosphorylation of the TEY loop. Failure to inactive MAPK signaling can lead to developmental defects and progression of certain cancers [219]. Dually-phosphorylated ERK1/2 on T202 and Y204 can be inactivated in one of two ways. Dual-specificity phosphatases (DUSPs) can simultaneously remove both phosphate marks [220]. Alternatively, protein tyrosine phosphatases (PTPs) and protein serine phosphatases (PSPs) dephosphorylate tyrosine and serine/threonine residues, respectively [221,222]. Since aconitase inhibition correlates with increased steady-state p-ERK1/2 levels, the possibility of defective dephosphorylation as a mechanistic cause for increased ERK activation was investigated.

Increased MKP-3 levels under aconitase inhibition

Among the family of DUSPs, also called MAP Kinase Phosphatases (MKPs), DUSP6/MPKP-3 was of interest because it is an ERK1/2-specific cytosolic MKP, as opposed to MKP-1 and MKP-2, who act in the nucleus on ERK1/2, p38, and JNK [219,223]. MKP-3 expression can be regulated transcriptionally; this appear to be celltype specific. MKP-3 is also regulated post-transcriptionally. Phosphorylation of MKP3 in a MEK-ERK dependent fashion promotes its degradation by the proteasome [224]. 
Figure 3.8 Increased MKP-3 total protein in FA-treated erythroid progenitors. $\mathrm{CD} 4^{+}$progenitors were cultured in erythroid medium $\pm 50 \mu \mathrm{M}$ FA for $2-4$ days. Protein levels of MKP-3, p-ERK1/2, and ERK1/2 were assessed by immunblotting of whole cell lysates. Tubulin is shown as a loading control. 
Figure 3.8 Increased MKP-3 total protein in FA-treated erythroid progenitors.

\begin{tabular}{|c|c|c|c|c|c|c|}
\hline \multicolumn{2}{|c|}{ Day 2} & \multicolumn{2}{|c|}{ Day 3} & \multicolumn{2}{|c|}{ Day 4} & \\
\hline NT & FA & NT & FA & NT & FA & \\
\hline- & - & - & - & - & - & MKP-3 \\
\hline- & $=$ & $=$ & $=$ & $=$ & $=$ & p-ERK1/2 \\
\hline$=$ & $=$ & - & & & & ERK1/2 \\
\hline & & & & & & Tubulin \\
\hline
\end{tabular}


Total levels of MKP-3 were elevated in primary erythroid progenitors treated with FA (Figure 3.8). This increase was observed in several independent experiments and correlated each time with increased p-ERK1/2. While the levels of phosphorylated MKP-3 were not assessed due to lack of commercially available antibodies, the increase in total MKP-3 levels suggest that this phosphatase is not actively being degraded despite increased ERK1/2 activity.

\section{PP2A does not mediate the effects of FA in erythroid progenitors}

PP2A is an essential regulator of Raf-MEK-ERK signaling, with the ability to dephosphorylate all three kinases in this pathway [225-227]. PP2A is composed of three subunits, including a catalytic subunit, PP2Ac, whose activity can be regulated by methylation [228,229]. A small body of published data suggests that metabolic intermediates such as citrate can promote methylation of PP2Ac, a modification that inhibits PP2A activity and thus could conceivably maintain ERK1/2 in an activated state [229,230]. Since FA-mediated aconitase blockade causes an increase in citrate, inactivation of PP2Ac via citrate-induced methylation constituted an appealing mechanism. The methylation status of PP2Ac was evaluated in primary erythroid progenitors by immunoblotting. Chronic FA treatment did not affect the levels of methylated and demethylated PP2A (Figure 3.9).

As an alternative approach, the impact of pharmacological modulators of PP2A where compared to those of FA. 1,9-Dideoxy Forsoklin (DDF) is a potent activator of PP2A that does not activate adenylate cyclase, unlike its close relative forskolin 
Figure 3.9 Aconitase blockade does not affect methylation of PP2A catalytic

subunit. $\mathrm{CD}^{+} 4^{+}$progenitors were cultured in erythroid medium $\pm 50 \mu \mathrm{M}$ FA for $2-4$

days. Protein levels of methylated PP2Ac (me-PP2Ac) and demethylated PP2Ac (deme-

PP2Ac) were assessed by immunblotting of whole cell lysates. Tubulin is shown as a

loading control. 
Figure 3.9 Aconitase blockade does not affect methylation of PP2A catalytic subunit.

\begin{tabular}{|c|c|c|c|c|c|c|}
\hline \multicolumn{2}{|c|}{ Day 2} & \multicolumn{2}{|c|}{ Day 3} & \multicolumn{2}{|c|}{ Day 4} & \multirow[b]{3}{*}{ me-PP2A } \\
\hline NT & FA & NT & FA & NT & FA & \\
\hline & & & - & & & \\
\hline & & & & & & deme-PP2 \\
\hline & & & & & & Tubulin \\
\hline
\end{tabular}


[231,232]. Inhibition of PP2A activity can be achieved with low-dose okadaic acid (OA), a natural product isolated from shellfish [233]. Treatment of erythroid progenitor cultures with DDF or OA did not affect erythroid proliferation in a statistically significant manner, although there was a trend toward diminished proliferation with addition of OA (Figure 3.10A-B). Similarly, the PP2A modulators had no impact on the differentiation of erythroid progenitors. DDF and OA-treated cells were able to differentiate as well as untreated cells, as assessed by the percentage of cells expressing GPA on their surface (78\%, 72\% and 77\%, respectively) (Figure 3.10C). As previously described, FA-treated cells had diminished GPA expression (58\%). Neither DDF nor OA had adverse effects on cellular viability (Figure 3.10D). Thus, defective PP2A activity is unlikely to be the cause of the increase in ERK signaling observed in erythroid progenitors under chronic FA blockade. 
Figure 3.10 Pharmacological modulation of PP2A catalytic domain activity does not affect erythroid proliferation, differentiation, or viability. (A) Proliferation of CD34 cells cultured in erythroid medium $\pm 50 \mu \mathrm{M}$ FA or $\pm 10 \mu \mathrm{M}$ DDF. DDF, 1,9-Dideoxy Forskolin. ${ }^{*} P<0.05$ when comparing control and treated cells on day 5 of culture; $\mathrm{n}=$ 3. (B) Proliferation of CD $34^{+}$cells cultured in erythroid medium $\pm 50 \mu \mathrm{M}$ FA or \pm 10 nM OA. OA, Okadaic Acid. ${ }^{*} P<0.05$ when comparing control and treated cells on day 5 of culture; $n=3$. (C) Differentiation profiles of erythroid progenitors treated for 5 days with $\pm 50 \mu \mathrm{M} F A, \pm 10 \mu \mathrm{M}$ DDF or $10 \mathrm{nM}$ OA assessed by flow cytometry using GPA and CD34. (D) Viability of erythroid progenitors treated for 5 days with $50 \mu \mathrm{M}$ FA, 10 $\mu \mathrm{M}$ DDF, or $10 \mathrm{nM}$ OA. 
Figure 3.10 Pharmacological modulation of PP2A catalytic domain activity does not affect erythroid proliferation, differentiation, or viability.

A

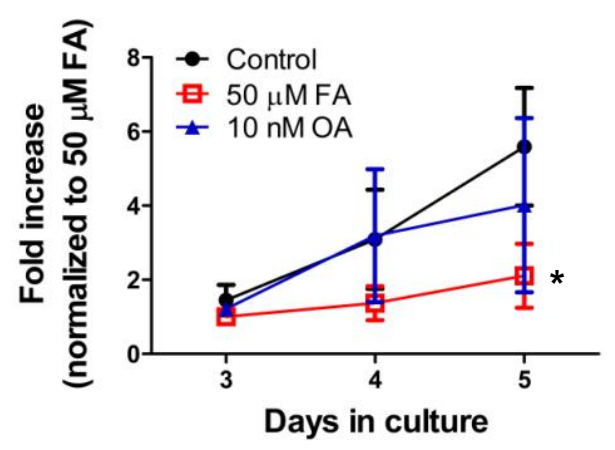

C
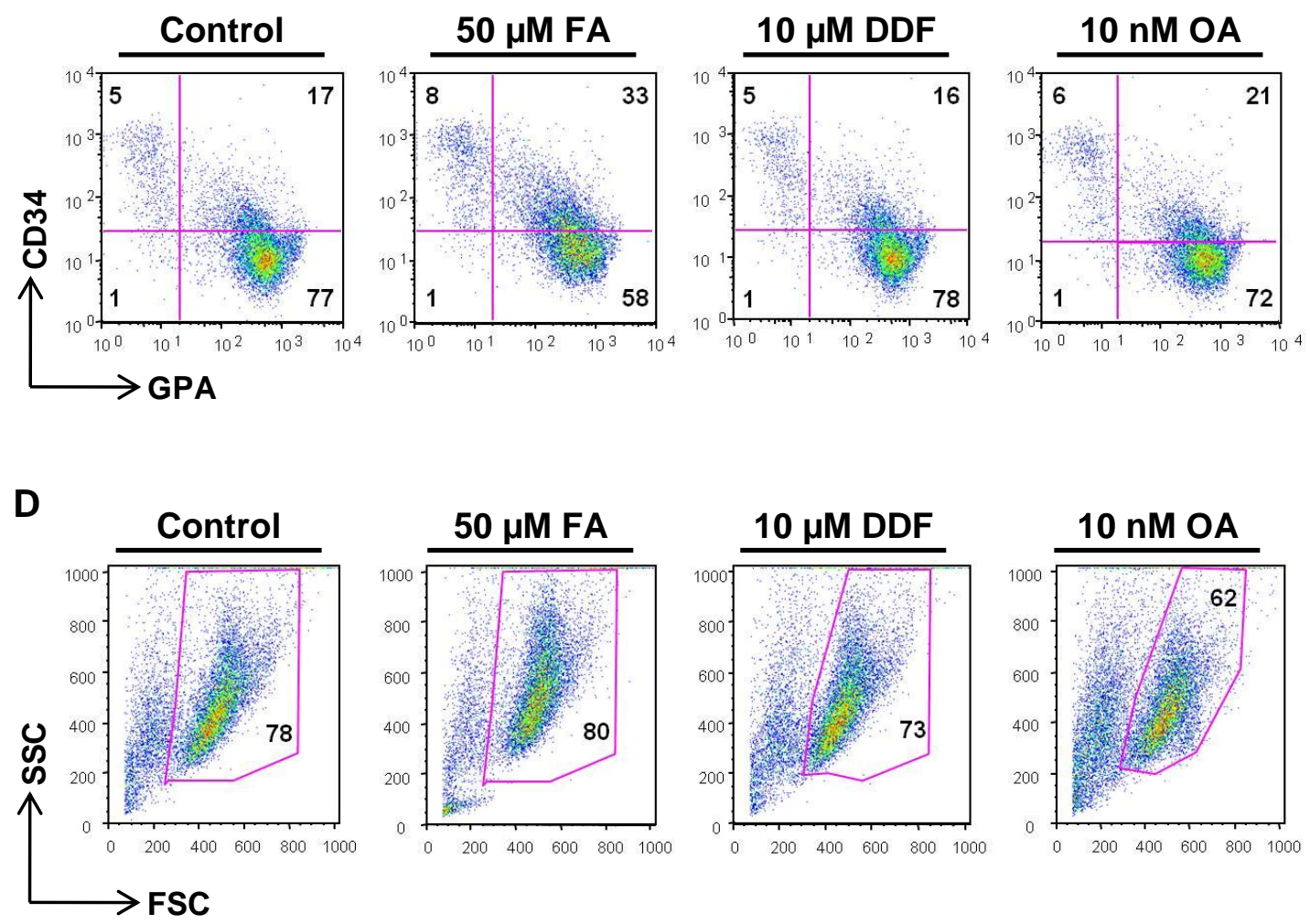

B

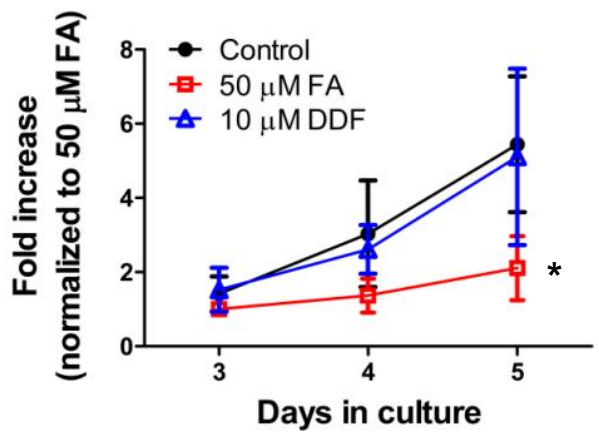




\section{$\underline{\text { Aconitase inhibition correlates with increased ERK1/2 in an insoluble cellular }}$}

\section{compartment}

Signaling studies in primary erythroid progenitors revealed that aconitase blockade impairs the activation of RSK, a cytosolic ERK1/2 target, but not that of Elk-1, a nuclear ERK1/2 target (Figures 2.7 and 3.6). These observations prompted the study of the subcellular distribution of total and phosphorylated ERK1/2. Erythroid progenitors were cultured for three days $\pm 50 \mu \mathrm{M}$ FA. The MEK inhibitor U0126 was added 30 minute prior to harvesting the cells. Cells were then fractionated into cytosolic, nuclear, and insoluble materials and analyzed by immunoblotting (Figure 3.10A). Purity of each fraction was assessed using superoxide dismutase 2 (SOD2), a mitochondrial protein, lactate dehydrogenase (LDH), a cytosolic protein, poly (ADP-ribose) polymerase (PARP), a nuclear DNA repair protein, and histone H3, a chromatin-associated protein. The majority of p-ERK1/2 and total ERK1/2 were found in the cytosol (Figure 3.11A, lanes 1-4). As expected, addition of U0126 reduced cytosolic p-ERK1/2 levels below the threshold of detection. Surprisingly, a significant amount of ERK1/2 was observed in the insoluble fraction with FA treatment (Figure 3.11A, lanes 9 vs. 11). This was unaffected by U0126 (Figure 3.11A, lanes 10 vs. 12). This insoluble fraction, characterized by the chromatin-associated protein histone $\mathrm{H} 3$, is likely to contain cytoskeletal components, histones, and large protein-associated complexes. These data suggest that ERK1/2 may participate in an insoluble complex (Figure 3.11A, lanes 9-10 vs. 11-12). 
These findings were confirmed in a separate experiment (Figure 3.11B). A portion of ERK1/2 co-fractionated with histone H3 upon FA treatment, regardless of U0126 (Figure 3.11B, lanes 8, 10 vs. 9, 12). Subcellular localization of phosphorylated and total Elk-1 were investigated as well, since acute EPO stimulation failed to dynamically regulate Elk-1 activation. Surprisingly, the majority of p-Elk-1 was located in the cytosol (Figure 3.11B, lanes 1-2, 5-6). Total Elk-1 was fairly evenly distributed between the cytosol and the nucleus (Figure 3.11B). U1026 treatment abrogated ERK1/2 activation but did not affect Elk-1 activation, thereby indicating that other protein kinases can phosphorylate Elk-1 (Figure 3.11B, lanes 1-4, 5-8). 
Figure 3.11 Increased levels of ERK1/2 in the insoluble compartment of fractionated FA-treated erythroid progenitors. (A) $\mathrm{CD} 34^{+}$cells were cultured for 3 days in erythroid medium $\pm 50 \mu \mathrm{M}$ FA. $10 \mu \mathrm{M}$ U0126 was added where indicated 30 minutes prior to harvest. The harvested cells were fractionated and each fraction was analyzed by immunoblot. SOD2, PARP, and histone H3 are loading controls for the cytosolic, nuclear, and insoluble fractions, respectively. NT, untreated; U, U0126. (B) Subcellular localization of activated and total ERK1/2 and Elk-1. Cells treated as in panel A were fractionated and analyzed by immunoblot. LDH, lactate dehydrogenase; SOD2, superoxide dismutase 2; PARP, poly (ADP-ribose) polymerase. 
Figure 3.11 Increased levels of ERK1/2 in the insoluble compartment of fractionated FA-treated erythroid progenitors.

A

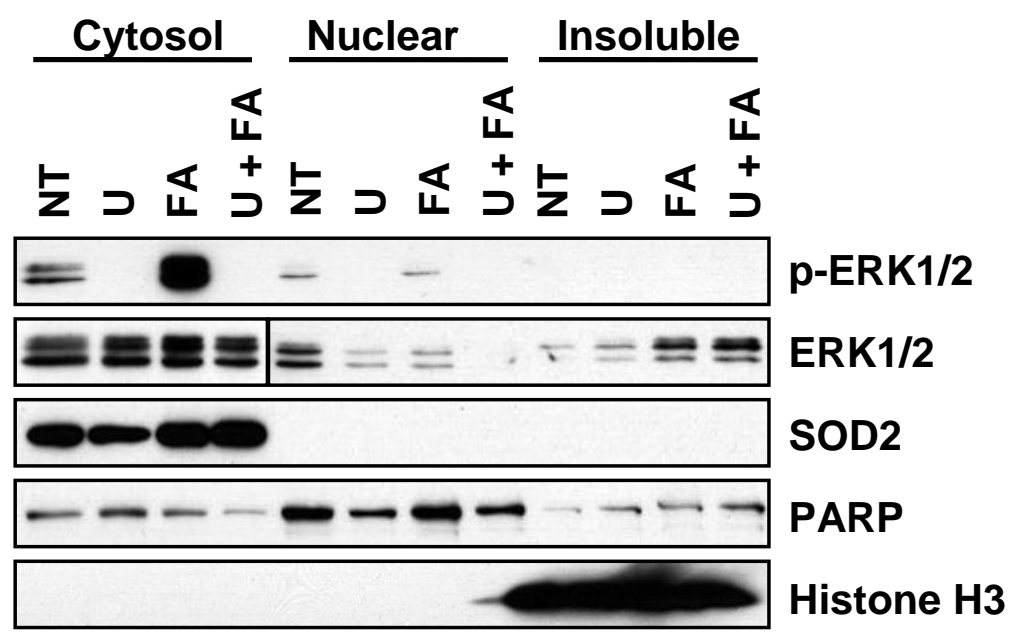

B

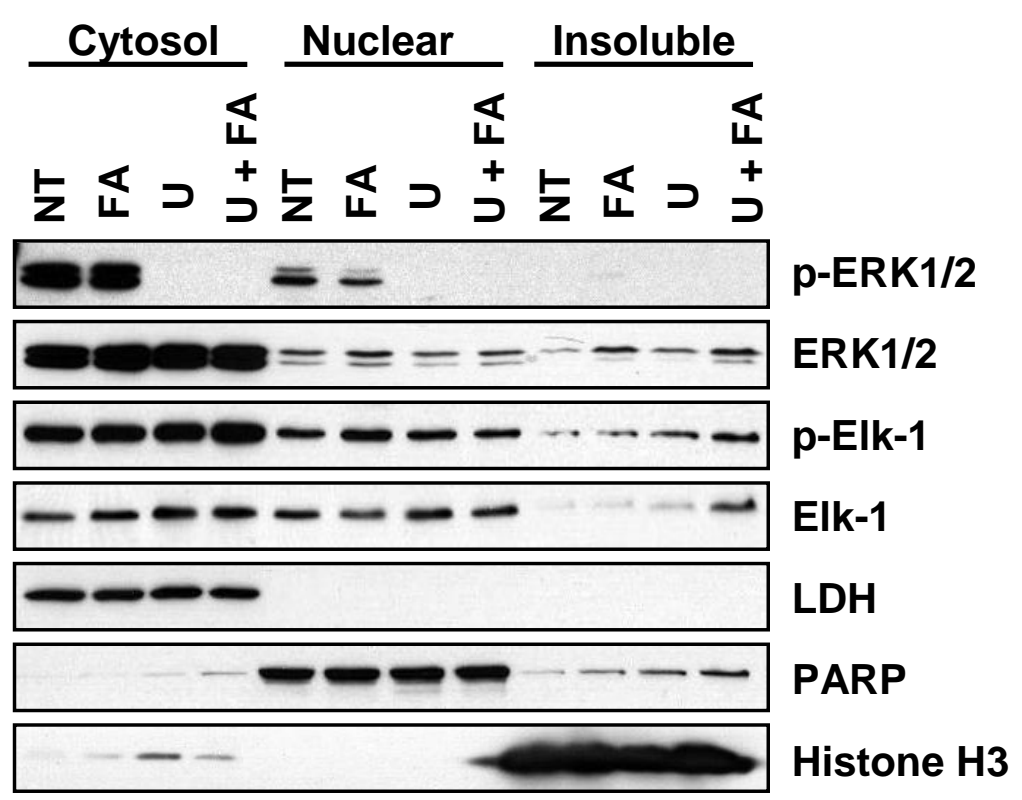




\section{EPO does not promote ERK2 dimer formation}

Despite the fact that FA did not modulate the formation of ERK2 dimers in HEK293T acutely stimulated with EGF (Figure 2.10), the status of ERK2 dimer formation in erythroid progenitor cells under acute EPO stimulation was investigated. Progenitors were cultured in erythroid medium $\pm 50 \mu \mathrm{M}$ FA for three days and starved of cytokines for 3 hours prior to EPO stimulation. As shown in Figure 3.12, ERK2 was found to exist exclusively in dimer form in $\mathrm{CD} 34^{+}$cells starved of cytokines. This is in contrast to EGF-starved HEK293T cells, where only monomeric ERK2 was detected (Figure 3.12, lanes 1 vs. 3). Neither EPO nor FA had any impact on ERK2 dimers. Thus, in erythroid progenitors, ERK2 exists exclusively in dimeric form and regulation of dimer assembly does not appear to be a relevant mechanism to control ERK signaling, as it is the case in HEK293T [188]. 


\section{Figure 3.12 ERK2 dimers are constitutively assembled in $\mathrm{CD34}^{+}$erythroid}

progenitors in the absence of EPO. Detection of ERK2 dimer formation upon EPO stimulation by native gel electrophoresis followed by immunoblotting. HEK293T cells were cytokine-starved overnight and stimulated with $100 \mathrm{ng} / \mathrm{ml}$ EGF for 5 minutes prior to harvest. $\mathrm{CD} 4^{+}$cells were cultured for 72 hours in erythroid medium $\pm 50 \mu \mathrm{M} \mathrm{FA}$, starved of cytokines for 3 hours, and stimulated with $4.5 \mathrm{U} / \mathrm{ml}$ EPO for 10 minutes prior to harvest. 
Figure 3.12 ERK2 dimers are constitutively assembled in $\mathrm{CD}^{+}{ }^{+}$erythroid progenitors in the absence of EPO.

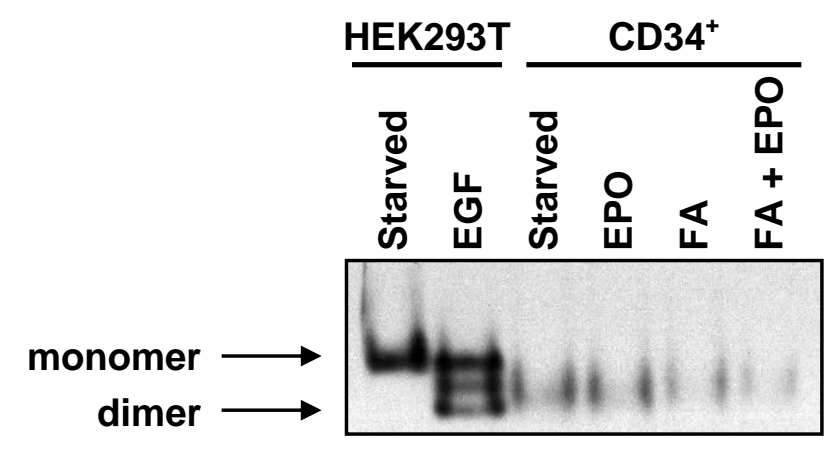




\section{A role for ERK1 in mediating the effects of FA in vivo}

Signaling and biochemistry studies have connected ERK1/2 signaling to aconitase function. ERK1/2 signaling to some downstream targets is perturbed when aconitase is inhibited by FA (Figure 2.7). To independently study a function for ERK during erythropoiesis under aconitase blockade, C57B1/6mice bearing a germline deletion of ERK1 (ERK1 -/- mice) were obtained [234]. ERK1 -/- mice were of interest because they have been recently shown to have elevated RBC parameters at baseline and to respond better to acute anemic stress [68]. Interestingly, only splenic erythropoiesis was altered in these animals, which led the authors to conclude that ERK1 plays a negative role during steady state splenic erythropoiesis. ERK1 -/- animals are viable, fertile, and histologically normal, but have defects in thymocyte maturation [235]. This is in stark contrast with ERK2 -/- animals, who die in utero at embryonic day E8.5-E11.5 due to defects in placental development $[69,236]$.

Adult ERK1 -/- mice and wild-type aged-matched mice received a four week infusion of $2 \mathrm{mg} / \mathrm{kg} /$ day FA and their response to aconitase blockade was compared. As shown in Figure 3.13A, ERK1 -/- mice baseline RBC count was not different from that of WT mice. Furthermore, both the hematocrit and the MCV of ERK1 -/- mice were lower than those of WT mice in a statistically significant manner (Figure 3.13B-C). These observations are in contrast to those of Guihard et al., who reported elevated RBC count, hematocrit, hemoglobin, and MCV in ERK1 -/- mice [68]. 
Figure 3.13 The red cell compartment of ERK1 -/- mice is partially resistant to the effects of FA. (A) RBC, (B) Hematocrit, (C) MCV, and (D) reticulocyte counts of ERK1 -/- and aged-matched WT mice treated with $2 \mathrm{mg} / \mathrm{kg} / \mathrm{FA}$ by continuous infusion for 28 days. Data is presented as mean $\pm \mathrm{SD} ; \mathrm{n}=8$ per group; ${ }^{*} P<0.05 ;{ }^{\dagger} P<0.01 ;{ }^{\ddagger} P$ $<0.001$. 
Figure 3.13 The red cell compartment of ERK1 -/- mice is partially resistant to the effects of FA.

A

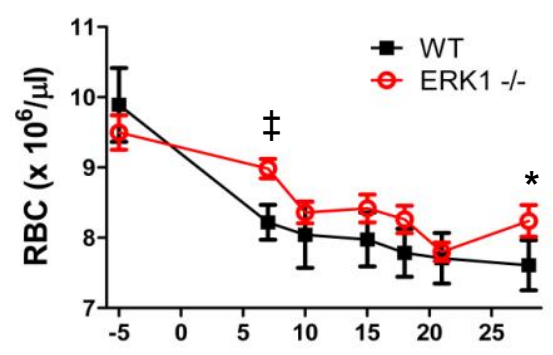

C

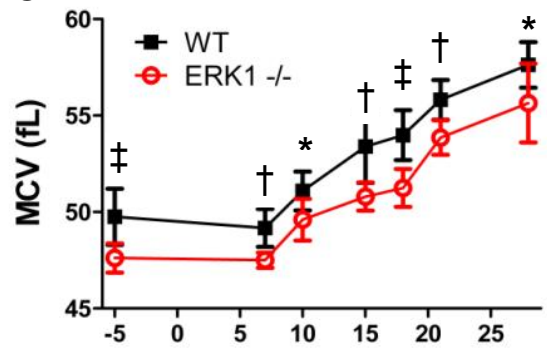

B
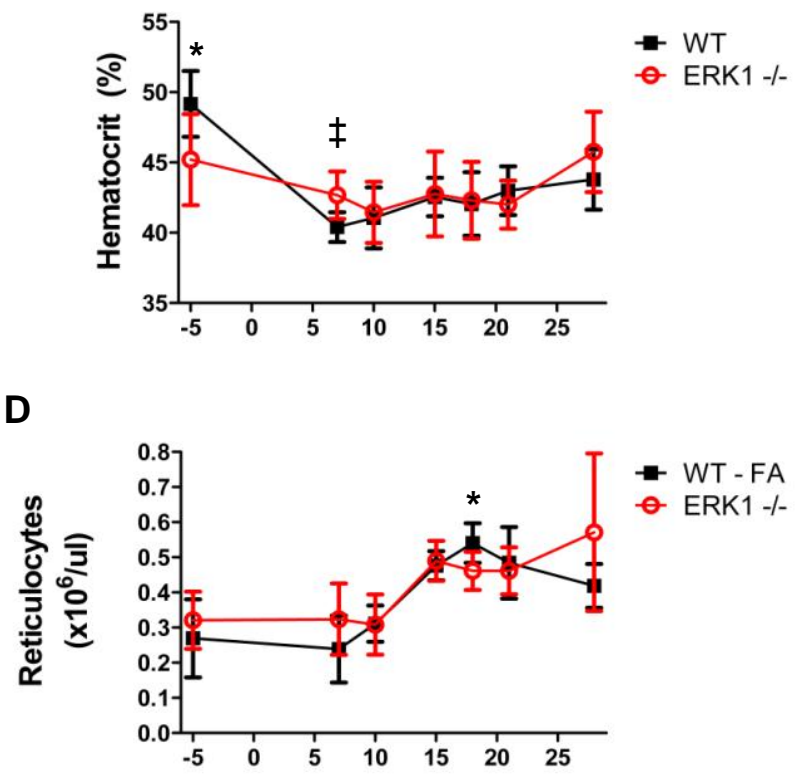
Table 3.1 Baseline platelet and white blood cell counts of WT and ERK1 -/- mice. Data are presented as mean \pm SD.

\begin{tabular}{lccc}
\hline & $\begin{array}{c}\text { WT } \\
(\mathbf{n}=8)\end{array}$ & $\begin{array}{c}\text { ERK1 -/- } \\
(\mathbf{n}=\mathbf{8})\end{array}$ & P value \\
\hline Platelets (k/ul) & $794 \pm 125$ & $819 \pm 113$ & 0.69 \\
Neutrophils (k/ul) & $0.88 \pm 0.29$ & $0.95 \pm 0.33$ & 0.65 \\
Lymphocytes (k/ul) & $6.55 \pm 1.82$ & $8.30 \pm 1.90$ & 0.08 \\
\hline
\end{tabular}

Table 3.2 Platelet and white blood cell count of WT and ERK1 -/- mice treated for four weeks with $2 \mathrm{mg} / \mathrm{kg} / \mathrm{day}$ FA. Data are presented as mean \pm SD.

\begin{tabular}{lccc}
\hline & $\begin{array}{c}\text { WT } \\
(\mathbf{n}=8)\end{array}$ & $\begin{array}{c}\text { ERK1 -/- } \\
(\mathbf{n}=\mathbf{8})\end{array}$ & P value \\
\hline Platelets (k/ul) & $960 \pm 104$ & $916 \pm 82.4$ & 0.36 \\
Neutrophils (k/ul) & $0.73 \pm 0.36$ & $0.82 \pm 0.45$ & 0.66 \\
Lymphocytes (k/ul) & $4.26 \pm 1.67$ & $3.31 \pm 1.21$ & 0.21 \\
\hline
\end{tabular}


There were no differences in platelet, neutrophil, and lymphocyte counts at baseline (Table 3.1). After 4 weeks of FA treatment ( $2 \mathrm{mg} / \mathrm{kg} /$ day $)$, the RBC counts of ERK1 -/mice were modestly but significantly higher than those of WT mice (Figure 3.12A). The hematocrit trended higher as well, but the data was not statistically significant (Figure 3.13B). The MCV of ERK1 -/- animals increased over time as is seen in WT mice, but remained smaller at all time points (Figure 3.13C). Reticulocyte counts were similar in both cohorts (Figure 3.13D). As is the case with WT mice, chronic FA infusion had no effect on platelet, neutrophils, and lymphocyte counts (Table 3.2).

Analysis of the bone marrow erythroid compartment at endpoint revealed small but significant differences between the two groups. Standard analysis using Ter119 and CD71 to resolve erythroid progenitors into 4 distinct subpopulations (R1-R4) did not show any differences between WT and ERK1 -/- progenitors (Figure 3.14A-B). This is consistent with the findings of Guihard and colleagues [68]. However, analysis of the Ter $119^{+}$compartment using CD44 and FSC revealed a decrease in the absolute number of progenitors in the R5 subpopulation (Figure 3.14C-D). R1 cells are considered the least mature, and R5 progenitors the most mature [175]. Thus ERK1 -/- status affects the impact of aconitase inhibition on the maturation profiles of erythroid progenitors in the bone marrow. 
Figure 3.14 Effect of aconitase inhibition on the bone marrow erythroid compartment of WT and ERK1 -/- mice. (A) Representative flow cytometry plots of marrow cells stained with CD71 and Ter119 are shown. Boxes indicate erythroid developmental stages R1-R4, from top left (R1) to bottom right (R4). (B) Summary of data from panel A displayed as absolute cell numbers per femur in regions R1-R4. Data is presented as mean $\pm \mathrm{SD} ; \mathrm{n}=4$ per group. (C) Representative flow cytometry plots of marrow cells stained with Ter119 and CD44. Subpopulations I-V are indicated. Numbers represent the percent cell present in each subpopulation. (D) Summary of data from panel $\mathbf{C}$ displayed as absolute cell numbers per femur in subpopulations I-V. Data is presented as mean $\pm \mathrm{SD} ; \mathrm{n}=4$ per group; ${ }^{*} P<0.05$. 
Figure 3.14 Effect of aconitase inhibition on the bone marrow erythroid compartment of WT and ERK1 -/- mice.

A
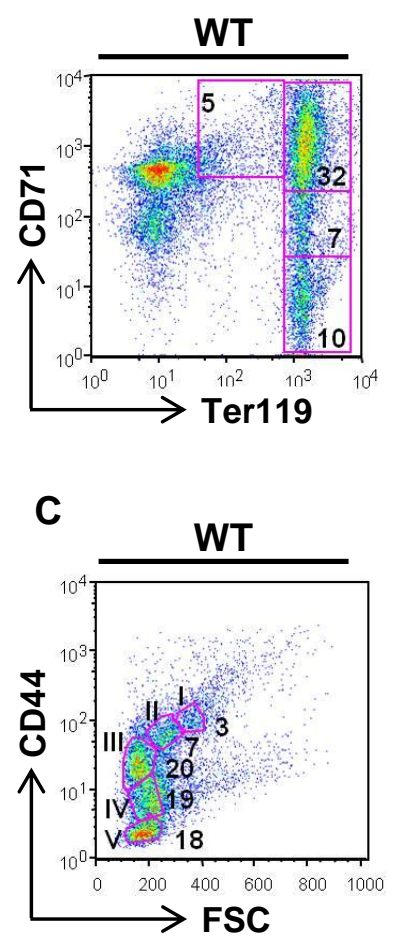

B
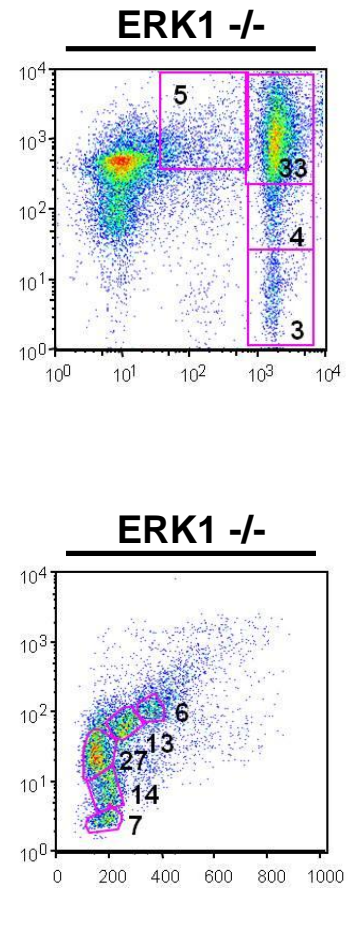

D
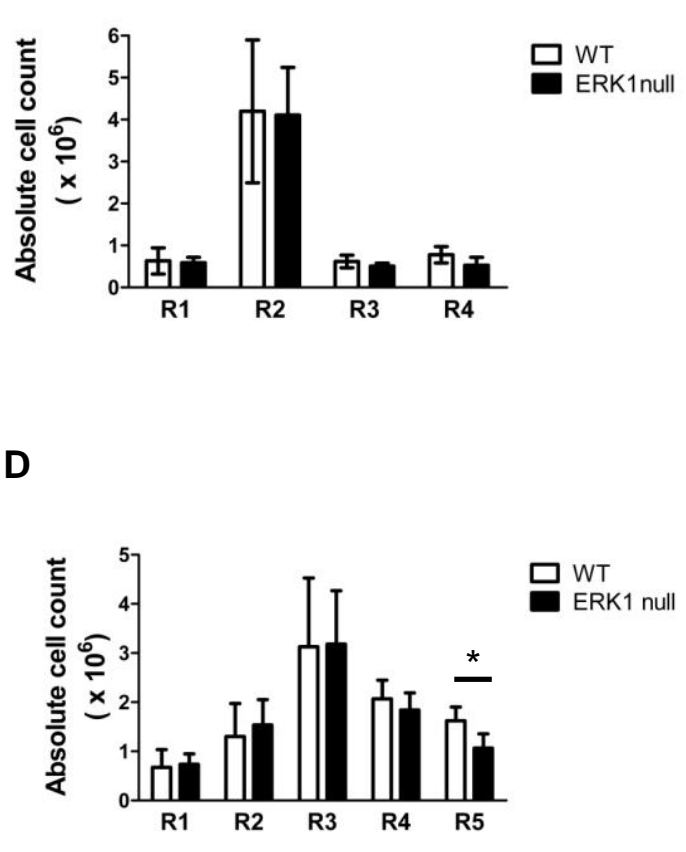


\section{$\underline{\text { Isocitrate does not rescue erythropoietic defects due to FA }}$}

Isocitrate, the metabolic product of the forward reaction catalyzed by aconitase, was shown to rescue erythroid defects caused by iron restriction in primary progenitors [87]. Since FA inhibits the active site of aconitase and prevents isocitrate synthesis, the ability of isocitrate to rescue FA-induced decreases in proliferation and differentiation was investigated. Erythroid cultures were treated with $50 \mu \mathrm{M} \mathrm{FA} \pm 20 \mathrm{mM}$ isocitrate, a dose shown to rescue differentiation [87]. As shown in Figure 3.15A, isocitrate did not increase the percentage of $\mathrm{GPA}^{+} \mathrm{CD} 34^{-}$cells. Furthermore, isocitrate had no effect of the levels of p-ERK1/2 and p-RSK observed with FA (Figure 3.15B).

The ability of isocitrate to modulate the anemia caused by FA infusion in vivo was also investigated. In a mouse model of dietary iron deficiency, intraperitoneal injections of $200 \mathrm{mg} / \mathrm{kg}$ isocitrate halted the progression of the anemia [87]. Mice were started on a $2 \mathrm{mg} / \mathrm{kg} /$ day FA infusion two weeks prior to receiving IP injections of isocitrate $(200 \mathrm{mg} / \mathrm{kg} /$ day $)$ or vehicle $(0.9 \%$ saline) for five consecutive days. The animals were bled prior to the first isocitrate injection, to assess the degree of FA-induced anemia, and again twenty four hours after the last isocitrate injection, to assess any potential correction in the RBC count. Isocitrate had no effect on the course of the RBC and reticulocyte counts when compared to vehicle (Figure 3.16A-B). The small upward shift in RBC numbers in both groups (Figure 3.16A) can be attributed to the onset of a modest reticulocyte response (Figure 3.16B). Thus, unlike in iron deficiency, isocitrate is unable to rescue FA-mediated erythroid defects in vitro and in vivo. 
Figure 3.15 Exogenous isocitrate does not reverse FA-mediated differentiation and signaling defect in primary erythroid cultures. (A) Differentiation of progenitors from day 5 erythroid cultures $\pm 50 \mu \mathrm{M}$ FA, $\pm 20 \mathrm{mM}$ isocitrate (IC). Surface expression of CD34 and GPA was assessed by flow cytometry on live cells; numbers indicate percentages of cells in each quadrant. (B) Induction of ERK1/2 and RSK phosphorylation by EPO. Cells from day 3 erythroid cultures $\pm 50 \mu \mathrm{M} \mathrm{FA} \pm 20 \mathrm{mM}$ isocitrate were subjected to 3 hours of cytokine starvation followed by stimulation with 4.5 U/ml EPO for ten minutes. 
Figure 3.15 Exogenous isocitrate does not reverse FA-mediated differentiation and signaling defect in primary erythroid cultures.
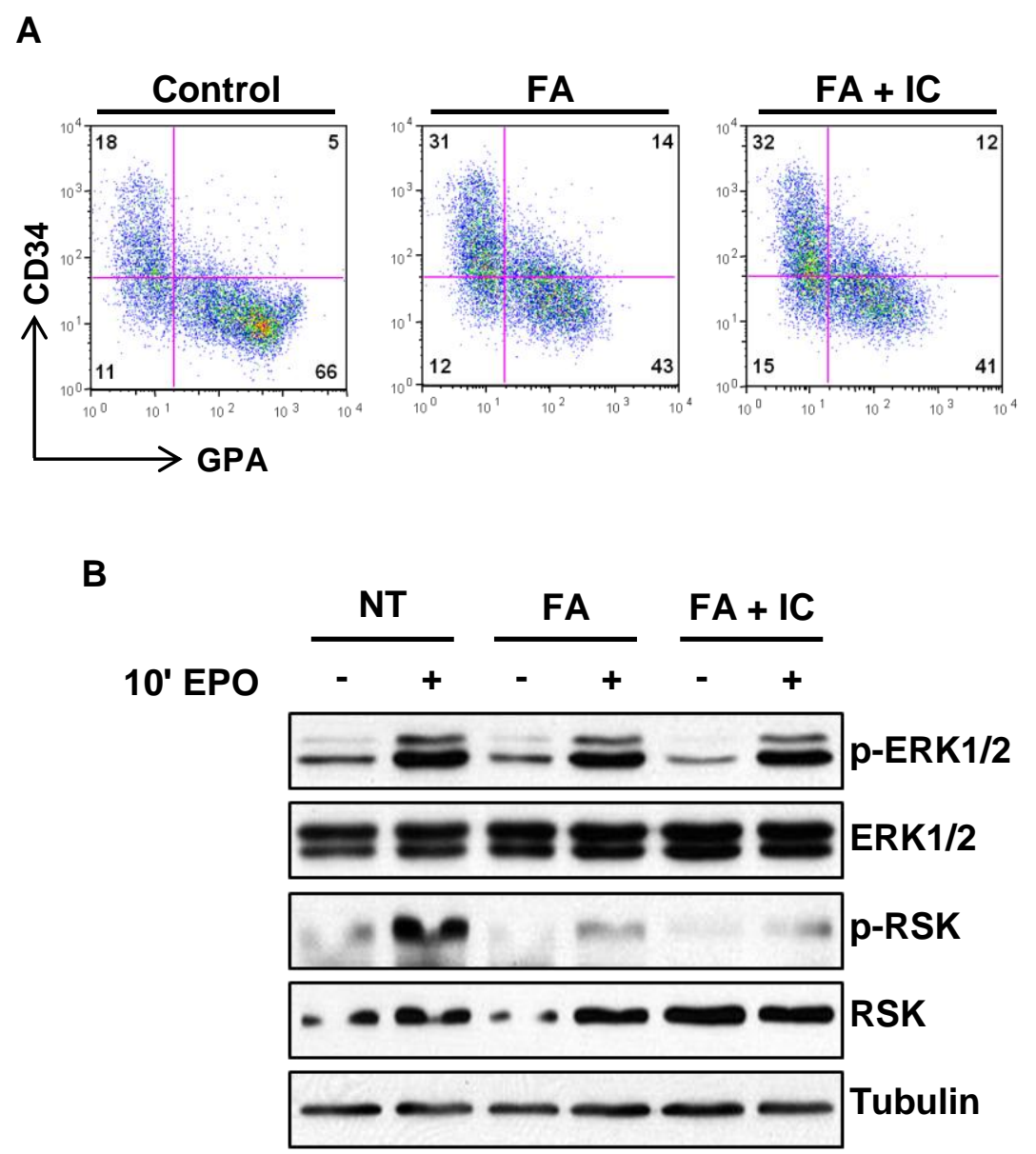
Figure 3.16 Isocitrate does not correct FA-induced anemia in wild type mice.

Evolution of the (A) RBC counts and (B) reticulocyte counts of wild type C57B1/6 mice who received a $2 \mathrm{mg} / \mathrm{kg} /$ day FA infusion and 5 consecutive IP injections of $200 \mathrm{mg} / \mathrm{kg}$

isocitrate or $0.9 \%$ saline on days $15-19$. Data is presented as mean $\pm \mathrm{SD} ; \mathrm{n}=8$ per group. 
Figure 3.16 Isocitrate does not correct FA-induced anemia in wild type mice.
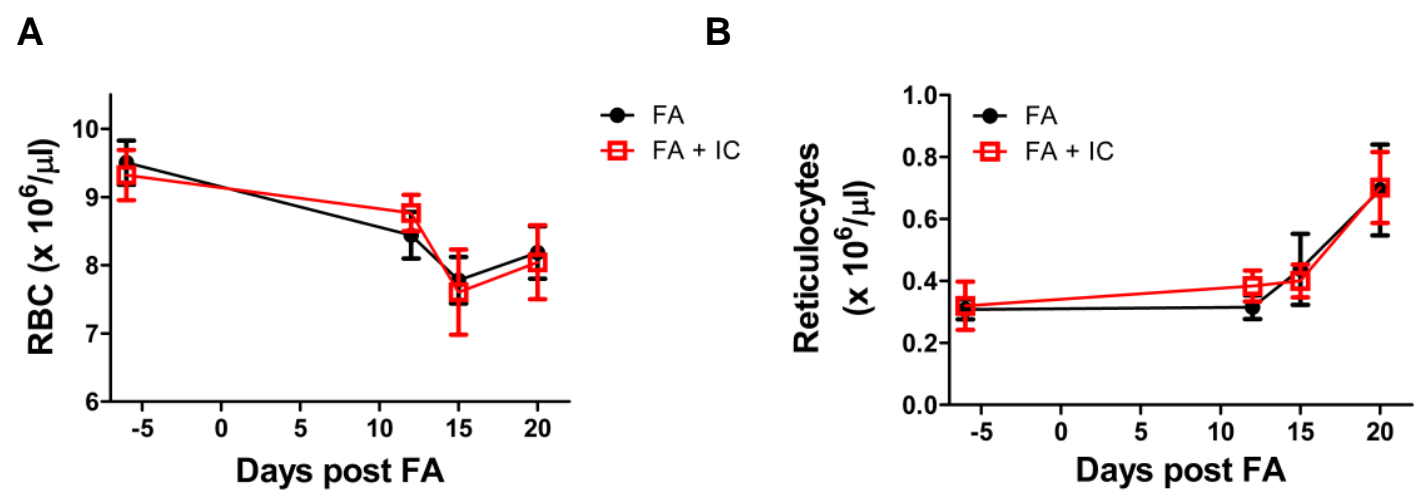


\section{Materials and Methods}

\section{Cell culture and reagents}

Peripheral blood mobilized, purified human $\mathrm{CD} 34^{+}$cells were obtained from the Hematopoietic Cell Processing Core at the Fred Hutchinson Cancer Center (Seattle, WA) [87]. After 72 hours in multi-lineage serum-free medium (prestimulation medium), the cells were seeded in serum-free erythroid differentiation medium as described in Chapter 2. SL0101 was purchased from Tocris (Ellisville, MO); Bi-D1870 from the Cohen Lab at the University of Dundee (Scotland, United Kingdom); isocitrate from Sigma; okadaic acid from Roche (Indianapolis, IN); 1,9-dideoxy forskolin from Biomol-Enzo Life Sciences (Farmingdale, NY).

\section{Plasmids}

pKH3 RSK2, pKH3 RSK2 K100A and pKH3 RSK2 Y707A were obtained from Dr. Deborah Lannigan (University of Virginia, Charlottesville, VA). pEYFP was from Clontech (Mountain View, CA), pcDNA3.1(+) from Invitrogen (Carlsbad, CA).

\section{Nucleofection}


$\mathrm{CD}_{3} 4^{+}$cells cultured in prestimulation medium for 48 hours were transfected using the Amaxa nucleofection technology (Lonza, Walkersville, MD). Briefly, 1 to $1.8 \times 10^{6}$ cells resuspended in $100 \mu \mathrm{L}$ of nucleofection solution provided in the Amaxa human CD34 cell nucleofector kit were combined with $4 \mu \mathrm{g}$ total DNA, transferred to the provided cuvettes, and electroporated using program U-08. The DNA consisted of $3 \mu \mathrm{g}$ of each expression construct and $1 \mu \mathrm{g}$ of the pEYFP-C1 marker plasmid (Clontech, San Diego, CA). The pcDNA3.1(+) plasmid was used as an empty vector control. Immediately after electroporation, cells were transferred to $3 \mathrm{~mL}$ of prewarmed erythroid differentiation medium in a six-well plate followed by culturing for the indicated durations.

\section{SiRNA knockdowns}

Knockdown of RSK1 and RSK2 in primary erythroid cells employed ON-TARGETplus small interfering RNA (siRNA) SMARTpool against either human RSK1 (L-003025-00) or human RSK2 (L-003026-00) purchased from Thermo Scientific Dharmacon (Lafayette, CO). A non-targeting siRNA was used as a control (D-001810-10). $0.8 \times 10^{5}$ $\mathrm{CD} 4^{+}$cells were maintained in erythroid medium for 24 hours prior to transfection with up to $100 \mathrm{nM}$ siRNA using TransIT-TKO reagent (Mirus Bio LLC, Madison, WI), according to the manufacturer's instructions (day 1). Cells underwent a second round of transfection the following day (day 2) and were allowed to differentiate in erythroid medium for an additional 48 hours (day 4). 


\section{Flow Cytometry}

Cells were analyzed as described in Chapter 2.

\section{Immunoblot}

Immunblot analysis was performed as described in Chapter 2. Antibodies against Elk-1, p-Ser388-Elk-1, PARP, and SOD2 were from Santa Cruz Biotechnologies. Anti-LDH was from Cell Signaling Technologies, anti-pSer228/231-MEK1/2 from Epitomics, and anti-histone $\mathrm{H} 3$ from Abcam (Cambridge, MA)

\section{Subcellular fractionation}

Untreated and $50 \mu \mathrm{M}$ FA-treated day 3 erythroid cultures were harvested and fractionated using the NE-PER Nuclear and Cytosolic Extraction Kit according to the manufacturer's instructions (Thermo Scientific, Waltham, MA). Cultures were also treated with $10 \mu \mathrm{M}$ U0126 prior to harvest as indicated. Extracted materials were resolved by SDS-PAGE electrophoresis and analyzed by immunoblotting.

\section{Mice}

ERK1 -/- mice were kindly provided by Dr. Gary Landreth (Case Western Reserve University, Cleveland, OH). Aged-matched wild type mice were purchased from Jackson 
Laboratories (Bar Harbor, ME). For continuous in vivo infusions, a sodium fluoroacetate (FA) solution prepared in $0.9 \%$ saline was delivered subcutaneously using Alzet osmotic pumps model 1004 (Durect) as described in Chapter 2. For isocitrate rescue experiment, adult female C57B1/6 mice were placed on a continuous FA infusion ( $2 \mathrm{mg} / \mathrm{kg} / \mathrm{day})$ and onset of anemia was monitor by retro-orbital eye bleeds. Isocitrate was dissolved in $0.9 \%$ saline to make a $200 \mathrm{mg} / \mathrm{kg}$ solution as previously described [87] and filtered through a $0.2 \mu \mathrm{m}$ filter. Isocitrate and $0.9 \%$ saline vehicle solutions were administered by intraperitoneal injections using 27 gauge tuberculin syringes (BD Biosciences). 
CHAPTER 4:

General Discussion 


\subsection{Aconitase activity regulates erythropoiesis}

\subsubsection{Inhibition of aconitase leads to decreased red cell production}

The central aim of this project was to investigate a role for aconitase during erythropoiesis. The data presented herein strongly show that aconitase activity modulates the production of red blood cells. This previously unappreciated function of aconitase is not secondary to global metabolic dysfunction but rather due to discrete effects on EPOdriven signaling events. Pharmacologic inhibition of aconitase activity impairs erythroid proliferation and maturation in vitro (Figure 2.7), and causes a dose-dependent normochromic, normocytic anemia in mice (Tables 2.1 and 2.2). In FA-treated mice, the reticulocyte count was decreased while EPO levels were elevated (Figure 2.2). The percentage of circulating reticulocytes is a direct indication of the rate of erythropoietic activity in the marrow [23]. Thus a low reticulocyte count suggests that the marrow was unable to ramp up erythroid production in FA-infused mice despite increased EPO stimulation. Analysis of the marrow erythroid compartment showed no absolute block in differentiation but instead an accumulation of cells at an intermediate stage of differentiation characterized by CD71 ${ }^{\text {intermediate }}$ Ter1 $19^{\text {bright }}$ staining intensities (Figures 2.2 and 2.11). A possible interpretation is that cells are slowed in their transit from a CD71 ${ }^{\text {intermediate }}$ Ter119 ${ }^{\text {bright }}$ phenotype to a more mature CD71 ${ }^{\text {dim }}$ Ter119 $9^{\text {bright }}$ phenotype, thereby resulting in less circulating red cells and anemia, as reflected in the CBC. The marrow Ter $119^{+}$erythroid progenitors was independently analyzed using a new scheme 
that employs the cell surface marker CD44 and FSC to tease out five subpopulations of erythroid progenitors, with $\mathrm{CD}_{4}{ }^{-} \mathrm{FSC}^{\text {low }}$ being the most differentiated of the five subgroups [175]. Marrows of FA-treated mice had significantly more cells per femur in the CD44-FSC ${ }^{\text {Low }}$ compartment (subpopulation V) (Figure 2.3D). This alternative approach to CD71 and Ter119 analysis has been touted as better able to resolve erythroid progenitors at distinct differentiation stages. While CD44 expression levels are distinct in the five subpopulations, there is overlap in CD71 expression levels, making it a less useful marker [175]. Furthermore, CD71 expression may be regulated by cellular iron levels via the IRP system, regardless of the differentiation stage of the erythroid progenitor [123]. Similarly, STAT5a/b can regulate mRNA levels of TfR1 [237]. It is difficult to evaluate whether the CD71 $1^{\text {intermediate }}$ Ter $119^{\text {bright }} \mathrm{R} 3$ population that is increased in FA-treated marrows corresponds to the CD44-FSC ${ }^{\text {Low }}$ subpopulation. Nevertheless, our data underscores the importance of conducting multiparametric FACS analysis when assessing erythroid maturation.

The notion of a generalized slowing of differentiation is further supported by in vitro data. FA-treatment of primary erythroid progenitors resulted in a significant proportion of cells expressing both CD34 and GPA, two markers thought to be mutually exclusive (Figure 2.5E-F). Cell cycle data showed partial accumulation of cells in $\mathrm{G}_{0} / \mathrm{G}_{1}$ with FA treatment, but no discrete cell cycle arrest (Figure 2.8B-C). It is tempting to speculate that a slow-down of the cell cycle causes the cells to proliferate and differentiate at a slower pace, resulting in aberrant co-expression of CD34 and GPA on the cell surface. In vivo, this may translate into cells spending more time at the 
CD71 ${ }^{\text {intermediate }}$ Ter $119^{\text {bright }}$ stage; a stage through which cells normally transit very rapidly. In support of this idea, studies investigating the consequences of chelator-mediated iron depletion in primary erythroid cells suggest that the decreased proliferation in iron deficiency is due to slowing of the cell cycle. Specifically, cells cultured in the presence of the iron chelator deferoxamine underwent three to four fewer rounds of cell division and were less like to be in S phase [238]. Another possibility is that aconitase inhibition regulates the lifespan of red blood cells, and that the anemia seen in vivo with FA infusion is due to increased turnover. In vitro characterization of the effects of FA revealed no increase in cell death or evidence of caspase-3 cleavage (Figure 2.5C), arguing against this mechanism. This could be tested in mock-treated and FA-treated mice by measuring red cell lifespan using a biotin-based approach [239].

\subsubsection{The erythroid lineage is highly sensitive to changes in aconitase activity}

An important finding of this study is that erythroid progenitors are exquisitely sensitive to a modest amount of aconitase inhibition. The dose of FA that robustly inhibited in vitro erythroid proliferation and differentiation, $50 \mu \mathrm{M}$, did not cause a drop in cellular ATP levels or activation of the energy sensor AMPK (Figure 2.6A-B). It is possible that progenitor cells compensated for decreased ATP synthesis via oxidative phosphorylation by upregulating glycolytic activity. This erythroid-specific sensitivity was confirmed in mice treated with a $4 \mathrm{mg} / \mathrm{kg} /$ day FA infusion, a dose that had a profound effect on red cell parameters, but inconsistent effects on white blood cell (Table 
2.1). A low $2 \mathrm{mg} / \mathrm{kg} /$ day dose was also found to be effective, with no discernable impact on platelet, neutrophil, and lymphocyte counts (Table 2.2). Collectively, these data argue that erythroid progenitors depend on a high threshold of aconitase activity and that partial inhibition of aconitase may target the erythroid compartment exclusively, an important consideration in thinking about potential therapeutic applications of this approach. In support of this notion, the murine erythroid G1-ER cell line arrested at the proerythroblast stage was also very sensitive to FA, with $10 \mu \mathrm{M}$ causing an increase in $\mathrm{p}$ ERK1/2 levels (not shown). This was not the case in non-erythroid cells lines. In HEK293T cells, a $500 \mu \mathrm{M}$ FA dose was necessary to significantly impair their proliferation (not shown), and the murine pro-B lymphocyte cell lines Ba/F3 EPOR and EPOR JAK2V617F were extremely resistant to the antiproliferative effects of FA (Figure 2.12A-B).

\section{$\underline{4.1 .3}$ Iron deficiency and FA blockade of aconitase}

We have previously reported on the impact of iron deficiency-mediated aconitase inactivation [87]. Similar to FA treatment, iron-restricted erythroid progenitor cultures were impaired in their proliferation and differentiation, two hallmarks of defective erythropoiesis. Iron restriction was associated with cell death, which was not seen with FA (Figure 2.5B). We did not observe cell cycle defects in erythroid progenitors cultured in low iron, but in one study, deferoxamine treatment reduced the percentage of erythroid progenitors in S phase $[87,238]$. Thus iron deficiency may have similar effects than FA 
on cell cycle kinetics, i.e. partial accumulation of cells in $\mathrm{G}_{0} / \mathrm{G}_{1}$ (Figure $2.8 \mathrm{~B}-\mathrm{C}$ ). Iron restriction in vivo caused a hypochromic, microcytic anemia, while FA blockade resulted in a normochromic, normocytic anemia (Table 2.1). Thus, the effects of iron deficiency and FA are not identical. This could be explained by the fact that iron restriction affects a large number of cellular processes while FA is specific for aconitase.

Isocitrate was shown to rescue erythroid differentiation in vivo and to correct anemia in a mouse model of dietary iron deficiency [87]. It seemed plausible that isocitrate could also reverse the effects of FA, either by bypassing the need for aconitase or by competing with FA for binding to the aconitase active site. Exogenous isocitrate failed to rescue FA-induced defects in maturation when used at a dose proven to restore maturation under low iron conditions (Figure 2.3.13). Similarly, intraperitoneal injections of $200 \mathrm{mg} / \mathrm{kg} /$ day isocitrate for five consecutive days in mice with documented reduction in red cell count due to ongoing FA treatment had no effect on red cell parameters (Figure 3.14). These data further argue that inactivation of aconitase by low iron and FA is not equivalent. Additionally, the failure of isocitrate to correct FAinduced anemia supports the notion that isocitrate functions in a non-metabolic way in IDA [87]. If that were the case, flooding of the cells with excess isocitrate should have corrected the FA-induced differentiation defect, at least partially. A recent body of literature supports non-traditional functions for metabolic intermediates. Wellen and colleagues have shown that acetyl CoA generated from citrate by ATP citrate lyase functions to regulate histone acetylation in response to growth factor stimulation [240]. In glioblastomas, gain of function mutations in isocitrate dehydrogenase 1 (IDH1) enable 
the enzyme to convert $\alpha$-ketoglutarate to 2 -hydroxyglutarate (2-HG), a metabolite known to be elevated in gliomas [241]. 2-HG was previously shown to accumulate in the central nervous system of patients with 2-hydroxyglutarate dehydrogenase deficiency; these patients are at an increased risk for brain tumors [242,243]. These new roles of acetyl CoA and 2-HG provide a link between metabolic status and differentiation and tumorigenesis, respectively. It is thus reasonable to conceive of isocitrate as a potential second messenger and of aconitase functioning as a nexus between metabolic status and differentiation signals during erythropoesis.

\subsection{A role for ERK signaling during erythropoiesis}

\subsubsection{Inhibition of ERK activation impairs erythropoiesis in vitro}

Published data posit both positive and negative roles for ERK signaling during erythroid development. Our studies argue that the outcome of ERK activation is not all positive or all negative, but rather the result of the integration of multiple activating and inhibitory signals. Initial observations suggested that ERK signaling had a positive effect on erythropoiesis. Chronic aconitase inhibition correlated with increased ERK1/2 phosphorylation without concomitant increase in RSK1 phosphorylation on a ERK1/2 target residue, threonine 573 [208,244] (Figure 2.7A). Docking of ERK1/2 onto the Cterminus of RSK1 and phosphorylation at T573 are believed to be the first steps leading to full activation of RSK1 [245]. When ERK signaling was induced with short term EPO 
stimulation after prolonged cytokine starvation, p-ERK1/2 levels in FA-treated cultures were similar to those in untreated cultures (Figure 2.7B-D). Again, aconitase inhibition correlated with severely decreased RSK1 activation (Figure 2.7B-D). Thus, aconitase inhibition specifically impairs ERK-mediated RSK activation under both chronic and acute EPO stimulus. The consequences of diminished RSK activation are discussed below (see Section 4.2.2.1). The increase in p-ERK1/2 levels seen with long term EPO and FA treatment, but not with short term EPO stimulation, may reflect a delayed compensatory mechanism in FA-treated cells whereby ERK1/2 activity is enhanced in an attempt to restore signaling downstream.

Inhibition of ERK activation by the MEK inhibitor U0126 reduced proliferation and differentiation of primary erythroid progenitors (Figure 2.8), also arguing for a positive role of ERK signaling during erythropoiesis. These data are in line with several previous studies. MEK inhibition was shown to inhibit formation of CFU-e colonies from wild type bone marrow extracts plated on semi-solid medium [66]. The negative effects caused by enforced ERK signaling secondary to constitutive Ras activation can be dampened by U0126 treatment, specifically restoring erythroid differentiation [46]. In erythroleukemic cells, expression of a constitutively active form of MEK1 inhibited $\gamma$ globin expression, but U0126 treatment reactivated gene expression and promoted synthesis of hemoglobin F [246]. In our studies, the negative effects of MEK inhibition on erythropoiesis paralleled those of FA, providing correlative evidence for a connection between aconitase inhibition and ERK signaling (Figure 2.8). 
A recently published study suggested that ERK1 is a negative regulator of erythropoiesis during stress erythropoiesis [68]. ERK1 -/- mice were treated with FA to assess the role of this ERK isoform in the response to aconitase blockade. We observed no differences between baseline red cell parameters of WT and ERK1 -/- mice, except for a statistically significant decrease in hematocrit and MCV (Figure 3.12). Since the hematocrit parameter takes into account the number and volume of the red cells, the low $\mathrm{MCV}$, rather than low red cell number, explained the lower hematocrit. These results differ from those reported by Guihard et al., who observed elevated RBC, hemoglobin, hematocrit, and MCV in their ERK1 -/- cohort at baseline [68]. It is possible that the smaller number of mice used in this study, 8 mice per group instead of 20 , precluded us from measuring statistically significant differences. However this in unlikely to be the case since our study was adequately powered to detect differences in hematocrit and $\mathrm{MCV}$. The ages of the animals may explain some the discrepancies observed on $\mathrm{CBC}$ at baseline. The study of Guihard et al. employed mice ranging in age from 2-4 months, while our cohort was 3 month-old at the start of the month-long experiment. Younger mice, i.e. 2-month old, may rely more heavily on ERK1 signaling. Sex-specific differences could also account for discrepancies on CBC. Our cohort was all female; the sex ratio of that of Guihard et al. is unknown. Lastly, differences in the approach chosen to generate the ERK1 -/- animals may explain the differences in baseline CBCs. The mice used in this study have a targeted deletion of exons 1-6 of the ERKl gene, eliminating expression of kinase subdomains I-X [234,247]. The study of Guihard and colleagues employed mice where exon 3 of the ERK1 gene was disrupted, such that 
truncated form of ERK1 protein containing exons 1 and 2 may still be expressed and retain function [235]. After a four week course of FA, ERK1 -/- mice had a slightly higher RBC count than the WT cohort, a statistically significant difference (Figure 3.12A). The hematocrit trended higher, but this was not significant, as was the case for the reticulocyte count (Figure 3.12B and C). These data suggest that ERK1 -/- mice were partially resistant to the negative effects of FA on red blood cells.

Thus, we obtained data supporting both positive and negative roles of ERK signaling during erythroid development. While seemingly contradictory, these findings suggest to us that downstream effectors of ERK signaling have pro-differentiation and anti-differentiation effects, and that the balance between these signals dictates outcome. Aconitase participates in the maintenance of this balance, the mechanism for which is not yet clear, and aconitase inhibition tips the balance in favor of diminished growth and maturation. Interestingly, U0126 treatment of erythroid progenitors and ERK1 knockout had opposite effects, with MEK inhibition impairing development and genetic knockout promoting resistance to an anemia-inducing drug. However, the latter approach is more targeted and preserved signaling via ERK2, while U1026 completely abrogated signaling by ERK1 and ERK2. These data may point to isoform-specific roles of ERK1 and ERK2 (see section 4.3.4). Further studies are needed to look comprehensively at the entire subset of downstream targets that are engaged upon ERK1/2 activation in erythroid progenitors. A phosphoproteomics approach would take an unbiased look at changes in the phosphorylation of all relevant ERK targets in erythroid cells. This technique would identify the subset of ERK targets relevant to erythropoiesis. After identification of 
candidate proteins, inhibitor studies with U0126 would be done to confirm that a particular target is relevant and would yield a manageable pool of ERK targets to further study. The next step would then look at how phosphorylation of these targets is modulated by aconitase inhibition. Such an approach was developed in HeLa cells to probe the phosphoproteome in response to acute EGF stimulation [248]. An interesting finding of that particular study was that while a large number of proteins are phosphorylated, only a small subset those phosphorylation events are sensitive to EGF stimulation. Because a phosphoproteomics approach is likely to yield a fairly large number of potentially relevant targets, further analysis could use gene set enrichment analysis to cluster ERK target genes that belong to known biological pathways [249].

\subsubsection{Signaling downstream of ERK1/2}

\subsubsection{RSK family of kinases}

Initial observations that aconitase blockade caused increased p-ERK1/2 levels in steady state conditions (Figure 2.7A) prompted the study of the activation status of downstream targets phosphorylated by ERK1/2. Survey of the literature indicated numerous nuclear targets for ERK1/2, including the transcription factor Elk-1 (See section 4.2.2.2). Activated ERK1/2 can also be retained in the cytoplasm and phosphorylate cytoplasmic targets, including the RSK family of kinases. These kinases were appealing candidates as potential mediators of the effects of FA during erythroid development because of their known role in proliferation, cell cycle regulation, and 
survival signaling [209,250-253]. An erythroid-specific role for RSK has not yet been ascribed, but RSK signaling is relevant to hematopoiesis. In erythroleukemic cells, activation of an ERK-RSK-NF- $\kappa$ B signaling axis by phorbol ester promoted megakaryocytic differentiation [254]. Two recent studies indicate that RSK activation, either by the tyrosine kinase receptor FGFR3 or the FMS-like tyrosine kinase 3 internal tandem duplication mutant, participates in hematopoietic transformation $[255,256]$.

A possible role for RSK signaling in erythropoiesis was investigated using several approaches. Collectively, our data in erythroid progenitors argue against a critical role for RSK signaling during erythroid differentiation. First, partial knockdown of both RSK1 and 2 did not affect erythroid differentiation (Figure 3.4). Second, overexpression of kinase-dead and constitutively active RSK2 mutants also had no impact on differentiation (Figure 3.3). Third, pharmacologic inhibition of RSKs yielded mixed results. The discrepancies observed between the two RSK inhibitors tested, SL0101 and Bi-D1870, remain unexplained. SL0101 significantly diminished both proliferation and differentiation while Bi-D1870 had a modest effect on growth and did not impair maturation (Figures 3.1 and 3.2 ). Both are ATP-competitive, reversible inhibitors, thought to be specific for RSKs. One study suggests that SL0101 is much less potent than Bi-D1870 [257]. It is possible that Bi-D1870 has a very narrow therapeutic window in $\mathrm{CD}_{3}{ }^{+}$cells, with $5 \mu \mathrm{M}$ being too low and $10 \mu \mathrm{M}$ too potent. A survey of the literature indicates that Bi-D1870 is well tolerated by cell lines at $10 \mu \mathrm{M}$ [258-260]. BiD1870 also inhibits Polo-Like Kinase 1 (PLK1) with almost equal potency than RSKs [257]. PLK1 is a protein kinase essential during mitosis, regulating entry into mitosis as 
well as centrosome assembly, cytokinesis, and more recently asymmetric cell division [261]. Although there are no published data on the role of PLK1 in erythroid development, it is possible that rapidly dividing erythroid progenitors have high levels of PLK1, causing Bi-D1870 to be toxic. SL0101 also inhibits the protein kinase Pim3, albeit somewhat less potently than RSKs [257]. Pim3 is a constitutively active kinase that promotes proliferation and has been associated with hematological malignancies [262]. Although the effects of SL0101 on RSKs have been extensively documented, it is possible that the defects observed in primary erythroid progenitor cultures are due to in part to Pim3 inhibition.

Further studies are needed to 1) implicate RSKs in erythroid development, 2) formally conclude whether RSKs are mediators or simply markers of aconitase-mediated erythropoiesis and 3) investigate the possibility of isoform-specific roles. Scanning of gene expression data deposited in the NCBI Gene Expression Omnibus database suggest that RSK1 and 2 are the predominantly expressed isoforms in human erythroid progenitors. Nevertheless, it would relevant to assess protein levels of all four RSK isoforms in these cells. Isoform-specific antibodies for each RSK are now commercially available. These studies may shed light on the RSK1 and RSK2 siRNA knockdown experiment, in particular if RSK3 and/or 4 are expressed in erythroid progenitors. One possibility is that RSK3 is able to compensate for diminished RSK1 and RSK2. Combined transient knockdown of RSK1 and RSK2 yielded approximately a 50\% reduction in the levels of each protein. It is possible that the residual kinase activity was sufficient to maintain RSK function. This could explain why the knockdown cells 
differentiated as well as control cells (Figure 3.4). Stable knockdown using retrovirallytransduced short-hairpin RNAs may yield stronger RSK knockdown and unmask important differences. Stable cells could also be assessed for growth over time, a parameter that cannot be measured in transiently transfected cells. Alternatively, the in vivo relevance of RSKs to erythropoiesis could be gathered from knockout mice. RSK2 knockout mice have been generated after mutations in human $R S K 2$ were found to cause Coffin-Lowry Syndrome (CLS), a neurological disease inherited in an X-linked dominant manner and characterized by mental retardation, small brain volumes, cardiovascular defects, and skeletal abnormalities [263,264]. RSK2 -/- mice show growth retardation as well as impaired learning and coordination [265]. Osteoclast differentiation defects in these animals have been subsequently reported [266]. Neither group has reported on the red cell parameters of these mice; CLS is not associated with anemia [264]. Triple RSK1/2/3 -/- animals have been generated and appear to be viable, but no phenotypic data has been reported [267]. It is possible that RSKs are dispensable during steady state erythropoiesis but necessary for the erythroid response to stress caused by aconitase inhibition, iron deficiency, or another stressor. Studying the effects of PHZ and FA in RSK -/- mice, either alone or in combination, as was done in WT mice, could help tease out important contribution of RSKs during erythropoiesis.

\subsubsection{Elk-1}

Elk-1 is one of the best characterized nuclear targets of ERK signaling. Elk-1, a member of the Ets family of transcription factors, is phosphorylated by ERK1/2 within 
minutes of stimulation and regulates gene transcription [268]. Among the genes regulated by Elk-1 is $c$-fos, an extensively studied effector of growth factor signaling [269]. The FA-mediated disruption in ERK signaling led us to hypothesize that Elk-1 activation may also be defective. This however was not the case. Robust p-Elk-1 levels were detected at baseline in cytokine-starved cells, and these levels were not modulated by acute EPO stimulation (Figure 3.6). While surprising at first, these results may be explained by the fact that multiple upstream kinases can phosphorylate Elk-1, including p38 and JNK [270,271]. Unlike p-ERK1/2 levels, FA did not affect the levels of p-p38 and p-JNK in erythroid progenitors (not shown). Thus it is conceivable that Elk-1 activation is carried out by $\mathrm{p} 38$ and/or JNK in erythroid progenitors. Additional control of phosphorylation of Elk-1 occurs via calcium-mediated activation of calcineurin, a phosphatase that can dephosphorylate Elk-1 regardless of the upstream kinase involved in its activation [272]. An alternative interpretation may be that the subcellular location of the ERK1/2 target dictates whether Elk-1 is able to be activated (see section 4.3.2).

\subsubsection{Evidence for MEK-independent activation of ERK1}

An unexpected finding of our study was that robust activation of ERK occurred without a corresponding increased in MEK activation (Figure 3.5). In fact, while cytokine starvation of erythroid progenitors resulted in barely detectable amounts of pERK1/2, p-MEK1/2 levels were detectable at baseline and did not change with short-term EPO treatment. These results raise several questions. First, how are basal p-MEK1/2 
levels maintained in the absence of growth factor stimulus? Second, since MEK is thought to be the only kinase able to phosphorylate ERK, how is the surge in p-ERK1/2 level achieved without increased MEK activation? Is there a previously unrecognized kinase capable of activating ERK?

Our observations that EPO stimulation following cytokine starvation does not dynamically regulate p-MEK1/2 levels are unexpected and do not concord with published data. In a study using starved primary erythroid progenitors (PEPs), Schmidt and colleagues showed increased p-MEK1/2 levels after 10 minutes of EPO [183]. Furthermore, the level of MEK1/2 activation was dependent on the dose of EPO used. A possibly explanation may reside in the manner in which the erythroid progenitors were derived. In our study, $\mathrm{CD} 34^{+}$multipotent progenitors were isolated from the peripheral blood of healthy donors who had received granulocyte-macrophage colony-stimulating factor injections to mobilize bone marrow progenitors (GM-CSF). These cells were expanded in multi-lineage supporting medium containing stem cell factor (SCF), thrombopoietin, Flt3-ligand, and IL-3 prior to a three day culture period in erythroidspecific serum-free medium supplemented with EPO and SCF. In contrast, Schmidt and colleagues used cord-blood derived $\mathrm{CD} 34^{+}$cells. Cells were initially expanded for 7 days in serum-free medium composed of IL-3, IL-6, and SCF, and separated by FACS based on $\mathrm{CD} 36$ expression. $\mathrm{CD}^{+} 6^{+}$cells were further cultured for 4 days in medium containing IL-3, IL-6, SCF, and EPO [183]. Thus not only the source of the progenitors, marrow vs. cord blood, but also the culture conditions varied significantly between the two studies and could account for the differences observed in response to EPO stimulation. The 
source of early progenitor cells and culture conditions are known to affect ex vivo expansion and marrow repopulation upon transplant [273,274]. Alternatively, MEKindependent ERK activation may be specific to bone marrow $\mathrm{CD} 34^{+}$progenitors.

Conventional activation of the Raf-MEK-ERK signaling cascade by a given growth factor is thought to proceed in a linear fashion, but an accumulating body of evidence strongly suggests that additional players can modulate MAPK signaling. In particular, PI3K and the classical PKCs are thought to be involved [183,275,276]. Sustained ERK signaling in response to Platelet-Derived Growth Factor stimulation was inhibited when cells were treated with the PI3K inhibitor Wortmannin [275]. In those studies, down-regulation of the classical PKCs had a similar effect. In the abovementioned study by Schmidt and colleagues, Wortmannin, as well as another unrelated PI3K inhibitor, LY294002, inhibited ERK1/2 phosphorylation in response to EPO stimulation [183]. In parallel, activation of MEK and ERK was detected in PEPs stimulated with low-dose EPO, despite pre-treatment with a Raf inhibitor. Inhibition of the classical PKCs, however, impaired MEK and ERK activation with low dose EPO. From these data the authors concluded that PI3K, and possible PKC, can activate MEK and ERK in a Raf-independent manner [183]. Specifically, PI3K $\gamma$ seems to the primary P3IK isoform capable of Raf-independent MEK/ERK activation. This was elegantly suggested by using caffeine, a PI3K inhibitor that has little selectivity toward the $\gamma$ isoform. While phosphorylation of Akt was potently inhibited by low-dose caffeine, higher doses were required to prevent phosphorylation of ERK1/2. Thus a mechanism can be envisaged where by $\mathrm{PI} 3 \mathrm{~K}_{\alpha, \beta, \delta}$ function to activate Akt while PI3K $\gamma$ specifically 
acts on MEK and ERK activation. In our own studies, phosphorylation of Akt was robustly enhanced by EPO stimulation and was insensitive to the effects of aconitase inhibition (Figure 2.7C). It is conceivable that in the presence of FA, Raf-independent, PI3K $\gamma$-dependent ERK1/2 activation is inhibited, thereby resulting in decreased ERK1/2 signaling. Future experiments in our primary erythroid progenitor cultures could use caffeine in conjunction with FA. If PI3K $\gamma$ mediates some of the effects of FA, caffeine may further sensitize the cells to the aconitase inhibitor and worsen the growth and maturation defects. Alternatively, overexpression of a constitutively active form of the kinase may rescue some of the anti-proliferative effects of FA. Additional evidence for non-canonical activation of ERK signaling comes from studies looking at the role of peroxynitrite in MAPK signaling. Treatment of rat fibroblasts with peroxynitrite increased ERK activation in a MEK-independent manner [277]. The authors go on to show that this activation is dependent on $\mathrm{PKC} \alpha, \mathrm{PKC} \varepsilon$, and calcium, with translocation of the $\mathrm{PKC}$ isoforms to the plasma membrane following treatment with peroxynitrite. In summary, while there is no definite precedent for the seemingly MEK-independent activation of ERK seen in erythroid progenitors acutely stimulated with EPO, there are several lines of evidence supporting the existence of an alternative pathway, possibly involving PI3-kinase. It is worth mentioning however, that scaffolding, rather than an alternative signaling pathway, could explain changes in ERK activation without changes in MEK phosphorylation status. For instance, the recruitment of ERK1/2 to assembled pre-activated MEK1/2 complexes could be regulated by high EPO. According to such a model, in the absence of EPO, erythroid progenitors have constant low p-MEK1/2 and no 
p-ERK1/2 due to physical separation of the two kinases. With addition of EPO, ERK1/2 is actively recruited to poised p-MEK1/2 modules and a surge of p-ERK1/2 occurs. Evidence for such a model is minimal, but the well-documented presence of dedicated MAPK scaffolds in several cell types suggests such a mechanism could be in place. An alternative explanation may be that FA-mediated aconitase inhibition results in ROS accumulation, which could in turn lead to inactivation of cytosolic phosphatases responsible for ERK1/2 dephosphorylation. ROS have been shown to mediate oxidation of a critical cysteine residue in the catalytic site of phosphatases [278]. We, however, did not observe any changes in ROS levels in erythroid cultured treated with FA for several days and ruled out PP2A as a relevant player (Figures 2.6C, 3.8, and 3.9), making this mechanism less appealing.

\subsection{Mechanistic insights}

\section{$\underline{4.3 .1}$ A novel aconitase-ERK interaction}

The physical interaction between aconitase and ERK1/2 described herein is the first report of such an association in a mammalian system. The interaction was first reported in a high throughpout study in S. cerevisiae that employed mass spectrometry to define new protein complexes [279]. The functional consequences of aconitase interacting with the yeast homolog of ERK were not investigated. In HEK293T cells, both aconitase isoforms were found to interact with ERK2 (Figure 2.9A). The m- 
aconitase-ERK2 interaction was more robust and sensitive to FA. If the two proteins are capable of interacting directly, a domain of the m-aconitase sequence must serve as a docking site for ERK. Two unrelated ERK interacting-domains have been detected in multiple ERK targets; DEF domains and D-Domains [208,280]. Interestingly, Elk-1, the nuclear transcription factor, interacts with ERK via a DEF domain, while RSK, the cytosolic ERK target, uses a D-Domain. Since aconitase inhibition disrupted ERKmediated RSK activation, but not Elk-1, the possibility that aconitase inhibition specifically affected D-Domain-mediated interactions was appealing. Scanning of the protein sequence of $\mathrm{c}$ - and $\mathrm{m}$-aconitase revealed the presence of a consensus D-Domain in both isoforms. Co-immunoprecipitation experiments using an ERK2 mutant defective in D-Domain interaction (ERK2 D319N) did not, however, show defective interaction of this mutant with m-aconitase (Figure 2.9B). The ERK2 L198/232A mutant, unable to interact with DEF Domain-containing proteins, was bound to m-aconitase as well as wild type ERK2 (not shown). It is thus unclear presently how the interaction between aconitase and ERK is facilitated. The interaction may employ an alternate domain on aconitase. Not all ERK targets contain DEF or D-domains. An alternative explanation is that the interaction is indirect, and that an unidentified protein serves as a linker between aconitase and ERK. In HEK293T cells, the interaction did not rely on activation of ERK, since acute stimulation with EGF prior to harvest did not modulate the strength of the aconitase-ERK interaction (Figure 2.9B).

While investigating the significance of this novel aconitase-ERK interaction, 
Casar et al. published a study demonstrating a critical role for ERK dimerization onto protein scaffolds for the activation of cytosolic targets [188]. Failure of ERK to dimerize and activate cytosolic targets led to decreased proliferation. Enforced nuclear localization of ERK had no significant impact on growth. Based on these results, we hypothesized that aconitase may function as a dimerization platform for ERK in the cytosol and that FA bound to aconitase prevents dimerization. However, assessment of ERK2 dimers in HEK293T cells acutely stimulated with EGF showed no defect in dimerization when the cells were pre-treated with FA (Figure 2.10). Furthermore, in primary erythroid progenitors, the majority of ERK2 was found to be in the dimer configuration even when the cells were starved of cytokines (Figure 3.12). There was no further dimerization observed when EPO was added acutely. It would appear that ERK2 molecules exist almost exclusively as dimers in $\mathrm{CD} 34^{+}$cells, an important difference from HEK293T cells, where EGF stimulation induces robust dimerization. Collectively, these data suggest that aconitase and ERK are normally found in complex with each other in HEK293T cells. Further studies should include endogenous pull-down experiments in HEK293T cells. The interaction should also be tested in an erythroid model system. If $\mathrm{CD}_{3}{ }^{+}$cells are not amenable to these experiments due to their limited proliferative capacity, the murine cell line G1-ER may be a useful resource. This erythroid cell line harbors a gene fusion between the master erythroid regulator gene GATA-1 and the estrogen receptor and is arrested at the proerythroblast stage unless exogenous estrogen is added [18]. 


\subsubsection{Subcellular site of ERK activation: location matters}

ERK1/2 can interact with a large variety of targets located in various cellular compartments. In fact, subcellular localization provides an elegant mechanism to finetune the outcome of ERK activation. Early on, ERK1/2 was understood to shuttle between the cytosol and the nucleus, where key transcription factor targets reside. It has become very clear however that ERK1/2 can translocate to additional subcellular compartments, including mitochondria, endosomes, Golgi, and plasma membrane. ERK1/2 can be targeted to endosomes via interactions with MP1 and p14, lipid rafts via p18, and the Golgi the scaffold protein Sef $[169,170,281,282]$. Compartmentalization between cytosol and nucleus relies in part on $\beta$-arrestin-2 and PEA-15. The earlier acts as a scaffold for Raf-MEK-ERK activation, but also mediates targeting of G-coupled protein receptors to early endosomes, thereby restricting ERK1/2 to the cytosol [283,284]. PEA-15 is a small cytosolic protein that also sequesters ERK1/2 to the cytosol. Of particular relevance is recent work showing that PEA-15 specifically brings ERK1/2 and RSK2 together to promote RSK2 phosphorylation and activation of the transcription factor CREB [285]. This scaffolding function of PEA-15 may be regulated by phosphorylation. Importantly, PEA-15 did not enhance ERK1/2-mediated activation of other cytosolic targets such as Stathmin and MNK. Since we observed a severe defect in ERK to RSK signaling in FA-treated erythroid progenitors (Figure 2.7B), it is tempting to speculate that aconitase activity may also regulate ERK/RSK interactions, either at the level of, or in parallel to, PEA-15. It would be of interest to assess total and 
phosphorylated levels of PEA-15 in erythroid progenitors $\pm 50 \mu \mathrm{M}$ FA. PEA-15 -/- mice have phenotypic abnormalities, but show no differences in red cell parameters [286].

\subsubsection{A mitochondrial signaling complex?}

The interaction data presented herein argues that the mitochondrial isoform of aconitase principally interacts with ERK1 and ERK2 (Figure 2.9 and not shown). This is further supported by the in vivo data in mice lacking cytosolic aconitase. IRP1 -/- status does not affect the response to FA infusion or to PHZ-induced acute anemic stress (Figures 2.13 and 2.4C-D). While the co-immunprecipitation experiments do not rule out the possibility of a third, unidentified player mediating an indirect interaction between maconitase and ERK1/2, it is tempting to speculate that a portion of cytosolic ERK1/2 either resides in the mitochondria and is activated within that compartment or translocates to the mitochondria upon activation. ERK1/2 has been localized to mitochondria in many different cell types. Neuronal SH-S5Y5 cells treated with 6-Hydroxydopamine have increased levels of activated ERK2 located in the mitochondria, which correlates with increased mitophagy [287]. In HeLa cells, mitochondrially located ERK1 was found to interact with a variety of proteins, including histone proteins associated with the outer mitochondrial membrane, and mitochondrial transport proteins such as VDAC [172]. ERK1/2 is also present in murine heart mitochondria and activation of ERK by a PKA/MEK module regulates steroidogenesis in MA-10 Leydig cells [200,288]. At the time of writing, localization of ERK1/2 to the mitochondria in erythroid progenitors has 
not been reported. One approach to address this question would be to use immunofluorescence in primary erythroid progenitors to study the subcellular distribution of ERK1/2 under steady-state, starved, and acute EPO stimulation conditions. A variety of fluorescent markers, such as MitoTracker CMX ROS used in this study to assess mitochondrial membrane potential (Figure 2.6D), could be used to identify specific organelles. Since the interaction between m-aconitase and ERK1/2 was down-regulated by FA, immunofluorescence studies should include FA-treated cells in parallel with untreated cells. Alternatively, subcellular fractionation using sucrose density gradient could be used to isolate mitochondria. The presence of ERK1/2 could be assessed in the collected fractions by immunoblotting. Raf -1 and MEK1/2 have also been shown to be targeted to the mitochondria [289,290]. In erythroid cells, it may be the case that aconitase serves as a scaffold to retain ERK1/2 in the mitochondria, and that mitochondrially-generated ERK1/2 signals are important for proliferation and differentiation. Disruption of this interaction by inhibiting the enzymatic activity of aconitase may therefore prevent ERK signaling to a small, specific subset of targets. It is unclear how FA disrupts this interaction. Our data suggest that the putative D-Domain of aconitase identified by scanning of its protein sequence does not participate in this interaction since an ERK2 D-Domain-interacting mutant, ERK2D319N, coimmunoprecipitated with aconitase (Figure 2.9). Lastly, ERK1/2 can interact with components of the cytoskeleton, mainly actin and microtubules. These interactions can be direct or rely on adaptor proteins such as calponin, IQGAP1, and LSP1 [291-293]. An intriguing function of the cytoskeleton in regulating MAPK signaling was demonstrated 
in embryonic stem cells, where addition of retinoic acid (RA) induced differentiation at the expense of proliferation by preventing activation of nuclear Elk-1 and c-fos while preserving activation of RSK in the cytosol [294]. Inhibitors of microtubule polymerization and actin filaments can re-activate c-fos-mediated transcription, indicating that the cytoskeleton acts to restrict nuclear entry of p-ERK1/2. This model is appealing and may be relevant to erythroid progenitors. Yeast interaction studies have identified two myosin motor proteins, MLC-1, a light chain for Myo1, and Myo4, as an interacting partner of Aco2, the yeast homolog of m-aconitase [295]. One model would posit that ERK-aconitase interactions with myosin help target activated ERK1/2 to downstream targets. In the presence of FA, aconitase is no longer able to bind ERK1/2 or myosin motors, which may results in mislocalization of activated ERK1/2 and diminished nuclear signaling, ultimately resulting in decreased proliferation. A role for the cytoskeleton in mediating some of the effects of FA could be investigated by immunofluorescence as well as inhibitor studies in the manner described by Smith and colleagues [294].

\subsubsection{ERK1 vs. ERK2}

An important future direction for this study will be to assess possible specific roles for ERK1 and ERK2 during erythropoiesis. The partial resistance to the effects of FA seen in ERK1 -/- mice (Figure 3.12) raises the possibility of isoform-specific contributions. According to Guihard and colleagues, levels of total and activated ERK2 
in the ERK1 -/- animals are similar to those of ERK1 +/+ animals, indicating that ERK2 does not compensate for lack of ERK1 and that the effects are indeed ERK1-specific [68]. In addition to the previously described dramatic differences between ERK1 -/- and ERK2 -/- animals (see Chapter 3), emerging data point to specific functions for ERK1 and ERK2. In rat hepatocytes, ERK2 is exclusively involved in cell cycle progression and proliferation [296]. ERK1, but not ERK2, regulates adipocyte differentiation in vitro and in vivo [297]. More generally, the proliferative effects of ERK signaling appear to be largely mediated by ERK2, while ERK1 has a negative effect on proliferation [298]. Ectopic expression of ERK1 impaired proliferation and transformation in NIH 3T3 fibroblasts co-expressing mutant H-Ras. ERK1 also attenuated Ras-dependent tumor formation in nude mice [298]. These data suggest that ERK1 modulate the final output of ERK activation by antagonizing ERK2 activity. Except for the work of Guihard and colleagues, it is unknown whether there are erythroid-specific functions of ERK1 and ERK2 [68]. Future studies could use the siRNA approach of Frémin et al. to study ERK1- and ERK2-specific roles in CD34 ${ }^{+}$erythroid cultures in response to a variety of stimuli, including EPO [296]. Inactivation of aconitase by FA or by iron deficiency could further help dissect the contributions of each isoform to erythroid development. Alternatively, ERK1 -/- splenic erythroblasts from ERK1-/-mice could be expanded ex vivo and transfected with shRNA against ERK2. Additionally, given that ERK1 may exert negative effects on proliferation, it would be of great interest to test the impact of ERK1 overexpression in a cell line with constitutive JAK2 signaling. Constitutively activated JAK2 does result in increased ERK1/2 activation [146]. This could be assessed 
by transiently overexpressing wild-type ERK1 or constitutively active MEK1 in Ba/F3 JAK2V617F cells. The kinase-deficient ERK1 K72R would provide adequate control. Alternatively, knockdown of ERK2 in Ba/F3 JAK2V617F could unmask the antiproliferative effects of ERK1. If ERK1 is shown to negatively regulate proliferation in the Ba/F3 JAK2V617F cell line, then crossing the ERK1 -/- mice with the PV mice should result in enhanced erythrocytosis and confirm ERK1 as a negative regulation of proliferation in red cell progenitors.

\subsubsection{A role for aconitase in unexplained anemia of the elderly?}

Multiple studies have linked erythroid dysplasia with mitochondrial dysfunction [196-198]. The underlying cause of mitochondrial dysfunction, however, can vary. Diwan and colleagues showed that defective autophagy secondary to disruption of the Nix gene in mice caused anemia and growth retardation [197]. Inoue et al. and Chen et al. independently provided strong evidence showing anemia in mice secondary to alterations in mitochondrial DNA $[175,196]$. Of particular interest is the fact that each group showed that the anemia was accompanied by an increase in the proportion of

CD71 ${ }^{\text {intermediate }}$ Ter $119^{\text {bright }}$ erythroid progenitors in the marrow. This finding is analogous to our own observations in FA-treated mice (Figure 2.2C and 2.11B), providing compelling evidence for a critical role of mitochondrial function in supporting bone marrow erythropoiesis. Whether aconitase function is specifically affected in these models of erythroid dysplasia is unknown but would be worth investigating. 
Mitochondrial dysfunction occurs naturally with aging. Accumulation of reactive oxygen species in particular has long been proposed to mediate some aspects of aging by interfering with essential mitochondrial functions such as cellular respiration [299]. Aconitase activity is very sensitive to ROS through its ISC (see section 1.4.2). Our work and that of others suggest that alterations in mitochondrial function are intimately associated with onset of anemia. Anemia in the elderly population was diagnosed in about $10 \%$ of individuals aged 65 or greater in a recent nation-wide study [300]. An underlying cause could not be ascertained for a third of these patients, and after bone marrow analysis, 25\% still have unexplained anemia (UA). A similar prevalence of UA in older patients was reported in at least two additional studies [301,302]. Anemia in the elderly is an independent risk factor for all-cause mortality [303]. Some studies suggest that unrecognized myelodysplastic syndromes (MDS) may explain a portion of UA cases [301,302]. MDS are age-related pathologies characterized in part by clonal abnormalities in primitive marrow progenitor cells, and mitochondrial dysfunction being increasingly considered as a possible disease mechanism [304]. Drugs known to impair mitochondrial protein synthesis, chloramphenicol and linezolid, can cause dyserythropoiesis [305,306]. Mutations in mitochondrial DNA have been detected in bone marrow cells isolated from MDS patients [307], and changes in transcription of mitochondrial genes in $\mathrm{CD}_{3} 4^{+}$cells of MDS patients have been reported [308]. Although m-aconitase is a nuclear-encoded gene, its function in the mitochondria may be perturbed in MDS and contribute to dyserythropoieisis. One could envision a mechanism whereby accumulation of mutations 
in mitochondrial DNA, along with ROS-mediated inactivation of proteins such as aconitase could reach a threshold level and contribute to impaired erythropoiesis.

\subsection{Clinical perspectives}

\subsubsection{Targeting aconitase in polycythemia vera}

Our studies suggest that direct modulation of aconitase activity can control erythroid production in mice harboring the mutant $J A K 2 V 617 F$ allele. FA infusion rapidly normalized RBC counts in PV mice to those of wild type mice mock-treated with saline (Figure 2.11A). Stable aconitase inhibition in the FA-infused mice was documented by an increase in serum citrate levels (Figure 2.1), suggesting that monitoring of citrate levels in the serum, or possibly in the urine, could be used clinically to assess response to treatment and titrate dosage. The current therapeutic standard for PV remains phlebotomy. While the exact mechanism explaining the effects of phlebotomy remains unclear, it is likely to be effective by inducing a state of functional iron deficiency following removal of a portion of circulating red cells. This acute depletion of iron, combined with the fact that reacquiring iron by dietary means takes time, blunts erythroid production, possibly by causing inactivation of ISC-containing enzymes such as aconitase. Thus, it may be possible to target aconitase and diminish the need for repeated phlebotomy, which would decrease the probability of undesired side 
effects, including reactive thrombocytosis. Indeed, FA-treated PV mice displayed no changes in their platelet counts (Table 2.3).

\subsubsection{Augmenting or preserving erythroid-specific ERK signaling to treat IDA}

A potential application of the research findings presented herein pertains to the treatment of anemia. Preserving aconitase function appears to be critical for erythroid development both in vitro and in vivo. Although exogenous isocitrate administration failed to rescue FA-induced anemia in mice, isocitrate has been shown to rescue irondeficiency anemia in cells and in mice [87]. Furthermore, primary erythroid progenitors cultured in low iron conditions show increased ERK activation, a defect that can be reversed by addition of isocitrate (not shown). Further dissection of the mechanism connecting aconitase activity to ERK signaling may identify key mediators of this pathway that could be targeted pharmacologically. Agonists of this pathway may help restore erythropoiesis and lessen the need for exogenous EPO injections. 


\subsection{Summary of future directions}

- Signaling studies

- Investigate further the contribution of RSKs to erythropoiesis

- Assess protein levels of RSK isoforms 1-4 in erythroid progenitor cultures

- shRNA knockdown of relevant RSK isoforms in $\mathrm{CD}_{3} 4^{+}$cells to assess impact on proliferation and maturation

- If RSK2 appears relevant in vitro studies, assess RSK 2 -/- mice for anemia after FA challenge and/or PHZ challenge

○ Role of PI3K

- Treat erythroid progenitor cultures with Wortmannin, LY, and caffeine

- Assess p-ERK1/2, p-RSK levels

- Assess differentiation by FACS using Ter119 and CD71

○ Phosphoproteomics screen

- Identify ERK1/2 targets modulated by EPO in erythroid progenitors

- Use gene set enrichment analysis to cluster targets into biologically relevant pathways to inform downstream analysis

- Validate targets in erythroid cultures pre-treated with U1026 prior to EPO stimulation

- Assess phosphorylation status of candidate targets by immunoblot

- Assess impact of FA on phosphorylation of candidate targets

- Aconitase-ERK interaction

- Investigate a role of PEA-15

○ Attempt endogenous reciprocal pull downs in HEK293T cells

- Attempt endogenous pull downs in erythroid progenitor cultures 
- Assess possible role of cytoskeleton in mediating the aconitase-ERK interaction

- Inhibitor studies with nocodazole and cytochalasin D

- ERK subcellularlocalization in erythroid progenitors

○ Immunofluorescence studies to establish ERK1/2, pERK1/2 subcellular localization

- Fractionation by sucrose density gradient to isolate mitochondria and probe for ERK via immunoblot

- Dissect the relative contributions of ERK1 and ERK2 to erythropoiesis

- Measure ERK2 mRNA and protein levels in ERK1 -/- mice

- Isolate ERK1 -/- splenic erythroblasts by cell sorting and culture ex vivo \pm FA.

- Develop mitochondrial aconitase knockout models

○ Culture m-aconitase -/- embryonic stem (ES) cells and assess viability and growth

- Induce differentiation of parental and m-aconitase -/- ES cells into embryoid bodies

- Generate m-aconitase -/- murine embryonic fibroblasts (MEFs) for biochemistry studies

- assess ERK signaling by immunoblot

- Compare proliferation rates of control MEFs and m-aconitase -/MEFs 
CHAPTER 5:

References 
1. Bryder D, Rossi DJ, Weissman IL (2006) Hematopoietic stem cells: the paradigmatic tissue-specific stem cell. Am J Pathol 169: 338-346.

2. Till JE, Mc CE (1961) A direct measurement of the radiation sensitivity of normal mouse bone marrow cells. Radiat Res 14: 213-222.

3. Weissman IL (2002) The road ended up at stem cells. Immunological Reviews 185: 159-174.

4. Zhu J, Emerson SG (2002) Hematopoietic cytokines, transcription factors and lineage commitment. Oncogene 21: 3295-3313.

5. Akashi K, Traver D, Miyamoto T, Weissman IL (2000) A clonogenic common myeloid progenitor that gives rise to all myeloid lineages. Nature 404: 193-197.

6. Kondo M, Weissman IL, Akashi K (1997) Identification of clonogenic common lymphoid progenitors in mouse bone marrow. Cell 91: 661-672.

7. Adolfsson J, Mansson R, Buza-Vidas N, Hultquist A, Liuba K, et al. (2005) Identification of Flt3+ lympho-myeloid stem cells lacking erythromegakaryocytic potential a revised road map for adult blood lineage commitment. Cell 121: 295-306.

8. Pevny L, Simon MC, Robertson E, Klein WH, Tsai S-F, et al. (1991) Erythroid differentiation in chimaeric mice blocked by a targeted mutation in the gene for transcription factor GATA-1. Nature 349: 257-260.

9. Tsang AP, Fujiwara Y, Hom DB, Orkin SH (1998) Failure of megakaryopoiesis and arrested erythropoiesis in mice lacking the GATA-1 transcriptional cofactor FOG. Genes \& Development 12: 1176-1188.

10. Elagib KE, Racke FK, Mogass M, Khetawat R, Delehanty LL, et al. (2003) RUNX1 and GATA-1 coexpression and cooperation in megakaryocytic differentiation. Blood 101: 4333-4341.

11. Vegiopoulos A, Garcia P, Emambokus N, Frampton J (2006) Coordination of erythropoiesis by the transcription factor c-Myb. Blood 107: 4703-4710.

12. Miller IJ, Bieker JJ (1993) A novel, erythroid cell-specific murine transcription factor that binds to the CACCC element and is related to the Kruppel family of nuclear proteins. Mol Cell Biol 13: 2776-2786. 
13. Olthof SG, Fatrai S, Drayer AL, Tyl MR, Vellenga E, et al. (2008) Downregulation of Signal Transducer and Activator of Transcription 5 (STAT5) in CD34+ Cells Promotes Megakaryocytic Development, Whereas Activation of STAT5 Drives Erythropoiesis. Stem Cells 26: 1732-1742.

14. Goldfarb AN (2007) Transcriptional control of megakaryocyte development. Oncogene 26: 6795-6802.

15. Shivdasani RA, Orkin SH (1996) The transcriptional control of hematopoiesis. Blood 87: 4025-4039.

16. Migliaccio AR, Rana RA, Sanchez M, Lorenzini R, Centurione L, et al. (2003) GATA-1 as a Regulator of Mast Cell Differentiation Revealed by the Phenotype of the GATA-1low Mouse Mutant. The Journal of Experimental Medicine 197: 281-296.

17. Yu C, Cantor AB, Yang H, Browne C, Wells RA, et al. (2002) Targeted Deletion of a High-Affinity GATA-binding Site in the GATA-1 Promoter Leads to Selective Loss of the Eosinophil Lineage In Vivo. The Journal of Experimental Medicine 195: 1387-1395.

18. Weiss MJ, Yu C, Orkin SH (1997) Erythroid-cell-specific properties of transcription factor GATA-1 revealed by phenotypic rescue of a gene-targeted cell line. Mol Cell Biol 17: 1642-1651.

19. Orkin SH, Zon LI (2008) Hematopoiesis: An Evolving Paradigm for Stem Cell Biology. Cell 132: 631-644.

20. Suda T, Suda J, Ogawa M (1983) Proliferative kinetics and differentiation of murine blast cell colonies in culture: evidence for variable $\mathrm{G}_{0}$ periods and constant doubling rates of early pluripotent hemopoietic progenitors. J Cell Physiol 117: 308-318.

21. Krause DS, Fackler MJ, Civin CI, May WS (1996) CD34: structure, biology, and clinical utility. Blood 87: 1-13.

22. Simpson CF, Kling JM (1967) The Mechanism of Denucleation in Circulating Erythroblasts. The Journal of Cell Biology 35: 237-245. 
23. Koury MJ, Koury ST, Kopsombut P, Bondurant MC (2005) In vitro maturation of nascent reticulocytes to erythrocytes. Blood 105: 2168-2174.

24. Lee JC, Gimm JA, Lo AJ, Koury MJ, Krauss SW, et al. (2004) Mechanism of protein sorting during erythroblast enucleation: role of cytoskeletal connectivity. Blood 103: 1912-1919.

25. Spivak JL (2005) The anaemia of cancer: death by a thousand cuts. Nat Rev Cancer 5: 543-555.

26. Socolovsky M, Nam H, Fleming MD, Haase VH, Brugnara C, et al. (2001) Ineffective erythropoiesis in Stat5a(-/-)5b(-/-) mice due to decreased survival of early erythroblasts. Blood 98: 3261 - 3273.

27. Bernard J (1991) The erythroblastic island: past and future. Blood Cells 17: 5-10.

28. Allen TD, Dexter TM (1982) Ultrastructural Aspects of Erythropoietic Differentiation in Long-term Bone Marrow Culture. Differentiation 21: 86-94.

29. Sadahira Y, Yoshino T, Monobe Y (1995) Very late activation antigen 4-vascular cell adhesion molecule 1 interaction is involved in the formation of erythroblastic islands. J Exp Med 181: 411-415.

30. Manwani D, Bieker JJ, James JB (2008) Chapter 2 The Erythroblastic Island. Current Topics in Developmental Biology: Academic Press. pp. 23-53.

31. Koury ST, Bondurant MC, Koury MJ (1988) Localization of erythropoietin synthesizing cells in murine kidneys by in situ hybridization. Blood 71: 524-527.

32. Lin FK, Suggs S, Lin CH, Browne JK, Smalling R, et al. (1985) Cloning and expression of the human erythropoietin gene. Proceedings of the National Academy of Sciences 82: 7580-7584.

33. D'Andrea AD, Lodish HF, Wong GG (1989) Expression cloning of the murine erythropoietin receptor. Cell 57: 277-285.

34. D'Andrea AD, Yoshimura A, Youssoufian H, Zon LI, Koo JW, et al. (1991) The cytoplasmic region of the erythropoietin receptor contains nonoverlapping positive and negative growth-regulatory domains. Mol Cell Biol 11: 1980-1987. 
35. Witthuhn BA, Quelle FW, Silvennoinen O, Yi T, Tang B, et al. (1993) JAK2 associates with the erythropoietin receptor and is tyrosine phosphorylated and activated following stimulation with erythropoietin. Cell 74: 227 - 236.

36. Damen JE, Mui AL, Puil L, Pawson T, Krystal G (1993) Phosphatidylinositol 3kinase associates, via its Src homology 2 domains, with the activated erythropoietin receptor. Blood 81: 3204-3210.

37. Klingmuller U, Bergelson S, Hsiao JG, Lodish HF (1996) Multiple tyrosine residues in the cytosolic domain of the erythropoietin receptor promote activation of STAT5. Proceedings of the National Academy of Sciences 93: 8324-8328.

38. Wu H, Liu X, Jaenisch R, Lodish HF (1995) Generation of committed erythroid BFU-E and CFU-E progenitors does not require erythropoietin or the erythropoietin receptor. Cell 83: 59-67.

39. Lin CS, Lim SK, D'Agati V, Costantini F (1996) Differential effects of an erythropoietin receptor gene disruption on primitive and definitive erythropoiesis. Genes \& Development 10: 154-164.

40. Parganas E, Wang D, Stravopodis D, Topham DJ, Marine J-C, et al. (1998) Jak2 Is Essential for Signaling through a Variety of Cytokine Receptors. Cell 93: 385395.

41. Sawada K, Krantz SB, Dai CH, Koury ST, Horn ST, et al. (1990) Purification of human blood burst-forming units-erythroid and demonstration of the evolution of erythropoietin receptors. Journal of Cellular Physiology 142: 219-230.

42. Quelle FW, Wang D, Nosaka T, Thierfelder WE, Stravopodis D, et al. (1996) Erythropoietin induces activation of Stat5 through association with specific tyrosines on the receptor that are not required for a mitogenic response. Mol Cell Biol 16: 1622-1631.

43. Wakao H, Gouilleux F, Groner B (1994) Mammary gland factor (MGF) is a novel member of the cytokine regulated transcription factor gene family and confers the prolactin response. EMBO J 13: 2182-2191. 
44. Teglund S, McKay C, Schuetz E, van Deursen JM, Stravopodis D, et al. (1998) Stat5a and Stat5b Proteins Have Essential and Nonessential, or Redundant, Roles in Cytokine Responses. Cell 93: 841-850.

45. Socolovsky M, Fallon AE, Wang S, Brugnara C, Lodish HF (1999) Fetal anemia and apoptosis of red cell progenitors in Stat5a-/-5b-/- mice: a direct role for Stat5 in Bcl-X(L) induction. Cell 98: 181 - 191.

46. Zhang J, Lodish HF (2004) Constitutive activation of the MEK/ERK pathway mediates all effects of oncogenic H-ras expression in primary erythroid progenitors. Blood 104: 1679-1687.

47. Jacobs-Helber SM, Ryan JJ, Sawyer ST (2000) JNK and p38 are activated by erythropoietin (EPO) but are not induced in apoptosis following EPO withdrawal in EPO-dependent HCD57 cells. Blood 96: 933-940.

48. Uddin S, Ah-Kang J, Ulaszek J, Mahmud D, Wickrema A (2004) Differentiation stage-specific activation of p38 mitogen-activated protein kinase isoforms in primary human erythroid cells. Proc Natl Acad Sci U S A 101: 147-152.

49. Jacobs-Helber SM, Sawyer ST (2004) Jun N-terminal kinase promotes proliferation of immature erythroid cells and erythropoietin-dependent cell lines. Blood 104: 696-703.

50. Huddleston H, Tan B, Yang FC, White H, Wenning MJ, et al. (2003) Functional p85alpha gene is required for normal murine fetal erythropoiesis. Blood 102: 142145.

51. Bouscary D, Pene F, Claessens YE, Muller O, Chretien S, et al. (2003) Critical role for PI 3-kinase in the control of erythropoietin-induced erythroid progenitor proliferation. Blood 101: 3436-3443.

52. Myklebust JH, Blomhoff HK, Rusten LS, Stokke T, Smeland EB (2002) Activation of phosphatidylinositol 3-kinase is important for erythropoietin-induced erythropoiesis from CD34(+) hematopoietic progenitor cells. Experimental Hematology 30: 990 - 1000. 
53. Myklebust JH, Smeland EB, Josefsen D, Sioud M (2000) Protein kinase C-alpha isoform is involved in erythropoietin-induced erythroid differentiation of CD34(+) progenitor cells from human bone marrow. Blood 95: 510 - 518.

54. von Lindern M, Parren-van Amelsvoort M, van Dijk T, Deiner E, van den Akker E, et al. (2000) Protein Kinase C alpha Controls Erythropoietin Receptor Signaling. Journal of Biological Chemistry 275: 34719-34727.

55. Liao H-J, Kume T, McKay C, Xu M-J, Ihle JN, et al. (2002) Absence of Erythrogenesis and Vasculogenesis in Plcg1-deficient Mice. Journal of Biological Chemistry 277: 9335-9341.

56. Tong Q, Hirschler-Laszkiewicz I, Zhang W, Conrad K, Neagley DW, et al. (2008) TRPC3 Is the Erythropoietin-regulated Calcium Channel in Human Erythroid Cells. Journal of Biological Chemistry 283: 10385-10395.

57. Gregoli PA, Bondurant MC (1997) The roles of Bcl-X(L) and apopain in the control of erythropoiesis by erythropoietin. Blood 90: 630-640.

58. Zang H, Sato K, Nakajima H, McKay C, Ney PA, et al. (2001) The distal region and receptor tyrosines of the Epo receptor are non-essential for in vivo erythropoiesis. EMBO J 20: 3156-3166.

59. Menon MP, Karur V, Bogacheva O, Bogachev O, Cuetara B, et al. (2006) Signals for stress erythropoiesis are integrated via an erythropoietin receptorphosphotyrosine-343-Stat5 axis. J Clin Invest 116: 683-694.

60. Barber DL, Corless CN, Xia K, Roberts TM, D'Andrea AD (1997) Erythropoietin Activates Raf1 by an Shc-Independent Pathway in CTLL-EPO-R Cells. Blood 89: $55-64$.

61. Johnson L, Greenbaum D, Cichowski K, Mercer K, Murphy E, et al. (1997) K-ras is an essential gene in the mouse with partial functional overlap with N-ras. Genes \& Development 11: 2468-2481.

62. Darley RL, Hoy TG, Baines P, Padua RA, Burnett AK (1997) Mutant N-RAS Induces Erythroid Lineage Dysplasia in Human CD34+ Cells. The Journal of Experimental Medicine 185: 1337-1348. 
63. Zhang J, Socolovsky M, Gross AW, Lodish HF (2003) Role of Ras signaling in erythroid differentiation of mouse fetal liver cells: functional analysis by a flow cytometry-based novel culture system. Blood 102: 3938-3946.

64. Uchida M, Kirito K, Shimizu R, Miura Y, Ozawa K, et al. (2001) A functional role of mitogen-activated protein kinases, erk1 and erk2, in the differentiation of a human leukemia cell line, UT-7/GM: a possible key factor for cell fate determination toward erythroid and megakaryocytic lineages. Int J Hematol 73: 78-83.

65. Fichelson S, Freyssinier J-M, Picard Fo, Fontenay-Roupie M, Guesnu M, et al. (1999) Megakaryocyte Growth and Development Factor-Induced Proliferation and Differentiation Are Regulated by the Mitogen-Activated Protein Kinase Pathway in Primitive Cord Blood Hematopoietic Progenitors. Blood 94: 1601-1613.

66. Abutin RM, Chen J, Lung TK, Lloyd JA, Sawyer ST, et al. (2009) Erythropoietininduced phosphorylation/degradation of BIM contributes to survival of erythroid cells. Experimental Hematology 37: 151-158.

67. Chen Z, Gibson TB, Robinson F, Silvestro L, Pearson G, et al. (2001) MAP Kinases. Chemical Reviews 101: 2449-2476.

68. Guihard S, Clay D, Cocault L, Saulnier N, Opolon P, et al. (2010) The MAPK ERK1 is a negative regulator of the adult steady-state splenic erythropoiesis. Blood 115: 3686-3694.

69. Hatano N, Mori Y, Oh-hora M, Kosugi A, Fujikawa T, et al. (2003) Essential role for ERK2 mitogen-activated protein kinase in placental development. Genes to Cells 8: 847-856.

70. Shaul YD, Seger R (2007) The MEK/ERK cascade: from signaling specificity to diverse functions. Biochim Biophys Acta 1773: 1213-1226.

71. Ebert BL, Bunn HF (1999) Regulation of the Erythropoietin Gene. Blood 94: 18641877.

72. Jaakkola P, Mole DR, Tian Y-M, Wilson MI, Gielbert J, et al. (2001) Targeting of HIF-1a to the von Hippel-Lindau Ubiquitylation Complex by O2-Regulated Prolyl Hydroxylation. Science 292: 468-472. 
73. Ivan M, Kondo K, Yang H, Kim W, Valiando J, et al. (2001) HIFa Targeted for VHL-Mediated Destruction by Proline Hydroxylation: Implications for O2 Sensing. Science 292: 464-468.

74. Maxwell PH, Wiesener MS, Chang G-W, Clifford SC, Vaux EC, et al. (1999) The tumour suppressor protein VHL targets hypoxia-inducible factors for oxygendependent proteolysis. Nature 399: 271-275.

75. Mastrogiannaki M, Matak P, Keith B, Simon MC, Vaulont S, et al. (2009) HIF2alpha, but not HIF-1alpha, promotes iron absorption in mice. The Journal of Clinical Investigation 119: 1159-1166.

76. Nicolas Gl, Chauvet C, Viatte L, Danan JL, Bigard X, et al. (2002) The gene encoding the iron regulatory peptide hepcidin is regulated by anemia, hypoxia, and inflammation. The Journal of Clinical Investigation 110: 1037-1044.

77. Yamashita T, Ohneda O, Sakiyama A, Iwata F, Ohneda K, et al. (2008) The microenvironment for erythropoiesis is regulated by HIF-2alpha through VCAM1 in endothelial cells. Blood 112: 1482-1492.

78. Peyssonnaux C, Zinkernagel AS, Schuepbach RA, Rankin E, Vaulont S, et al. (2007) Regulation of iron homeostasis by the hypoxia-inducible transcription factors (HIFs). J Clin Invest 117: 1926-1932.

79. Yoon D, Pastore YD, Divoky V, Liu E, Mlodnicka AE, et al. (2006) Hypoxiainducible Factor-1 Deficiency Results in Dysregulated Erythropoiesis Signaling and Iron Homeostasis in Mouse Development. Journal of Biological Chemistry 281: 25703-25711.

80. Hentze MW, Muckenthaler MU, Andrews NC (2004) Balancing Acts: Molecular Control of Mammalian Iron Metabolism. Cell 117: 285-297.

81. Collawn JF, Stangel M, Kuhn LA, Esekogwu V, Jing SQ, et al. (1990) Transferrin receptor internalization sequence YXRF implicates a tight turn as the structural recognition motif for endocytosis. Cell 63: 1061-1072.

82. Sheftel AD, Zhang A-S, Brown C, Shirihai OS, Ponka P (2007) Direct interorganellar transfer of iron from endosome to mitochondrion. Blood 110: 125-132. 
83. Forejtnikova H, Vieillevoye M, Zermati Y, Lambert M, Pellegrino RM, et al. (2010) Transferrin receptor 2 is a component of the erythropoietin receptor complex and is required for efficient erythropoiesis. Blood 116: 5357-5367.

84. Levy JE, Jin O, Fujiwara Y, Kuo F, Andrews N (1999) Transferrin receptor is necessary for development of erythrocytes and the nervous system. Nat Genet 21: 396-399.

85. Finch CA (1982) Erythropoiesis, erythropoietin, and iron. Blood 60: 1241-1246.

86. Kimura H, Finch CA, Adamson JW (1986) Hematopoiesis in the rat: quantitation of hematopoietic progenitors and the response to iron deficiency anemia. J Cell Physiol 126: 298-306.

87. Bullock GC, Delehanty LL, Talbot A-L, Gonias SL, Tong W-H, et al. (2010) Iron control of erythroid development by a novel aconitase-associated regulatory pathway. Blood 116: 97-108.

88. Vig M, Kinet J-P (2009) Calcium signaling in immune cells. Nat Immunol 10: 21-27. 89. Hensold JO, Dubyak G, Housman DE (1991) Calcium ionophore, A23187, induces commitment to differentiation but inhibits the subsequent expression of erythroid genes in murine erythroleukemia cells. Blood 77: 1362-1370.

90. Gillo B, Ma YS, Marks AR (1993) Calcium influx in induced differentiation of murine erythroleukemia cells. Blood 81: 783-792.

91. Miller BA, Scaduto RC, Tillotson DL, Botti JJ, Cheung JY (1988) Erythropoietin stimulates a rise in intracellular free calcium concentration in single early human erythroid precursors. The Journal of Clinical Investigation 82: 309-315.

92. Miller BA, Barber DL, Bell LL, Beattie BK, Zhang M-Y, et al. (1999) Identification of the Erythropoietin Receptor Domain Required for Calcium Channel Activation. Journal of Biological Chemistry 274: 20465-20472.

93. Hirschler-Laszkiewicz I, Tong Q, Waybill K, Conrad K, Keefer K, et al. (2011) The transient receptor potential channel TRPC3 TRP domain and AMP-actvated protein kinase binding site are required for TRPC 3 activation by erythropoietin. Journal of Biological Chemistry. 
94. Berridge MJ (2009) Inositol trisphosphate and calcium signalling mechanisms.

Biochimica et Biophysica Acta (BBA) - Molecular Cell Research 1793: 933-940.

95. Nagata S, Golstein P (1995) The Fas death factor. Science 267: 1449-1456.

96. Maciejewski J, Selleri C, Anderson S, Young NS (1995) Fas antigen expression on CD34+ human marrow cells is induced by interferon gamma and tumor necrosis factor alpha and potentiates cytokine-mediated hematopoietic suppression in vitro. Blood 85: 3183-3190.

97. De Maria R, Lenti L, Malisan F, d'Agostino F, Tomassini B, et al. (1997)

Requirement for GD3 Ganglioside in CD95- and Ceramide-Induced Apoptosis. Science 277: 1652-1655.

98. De Maria R, Testa U, Luchetti L, Zeuner A, Stassi G, et al. (1999) Apoptotic Role of Fas/Fas Ligand System in the Regulation of Erythropoiesis. Blood 93: 796-803.

99. Liu Y, Pop R, Sadegh C, Brugnara C, Haase VH, et al. (2006) Suppression of FasFasL coexpression by erythropoietin mediates erythroblast expansion during the erythropoietic stress response in vivo. Blood 108: 123-133.

100. De Maria R, Zeuner A, Eramo A, Domenichelli C, Bonci D, et al. (1999) Negative regulation of erythropoiesis by caspase-mediated cleavage of GATA-1. Nature 401: 489-493.

101. Beinert H, Kennedy MC, Stout CD (1996) Aconitase as Iron-Sulfur Protein, Enzyme, and Iron-Regulatory Protein. Chemical Reviews 96: 2335-2374.

102. Sparkes RS, Mohandas T, Sparkes MC, Shulkin JD (1978) Aconitase (E.C. 4.2.1.3) mitochondrial locus mapped to human chromosome 22: Studies with Chinese hamster-human somatic cell hybrids. Biochemical Genetics 16: 751-756.

103. Shows TB, Brown JA (1977) Mapping $A K_{1} A C O N_{s}$, and $A K_{3}$ to chromosome 9 in man employing an X/9 translocation and somatic cell hybrids. Cytogenetic and Genome Research 19: 26-37.

104. Srere PA (1959) The citrate cleavage enzyme. I. Distribution and purification. J Biol Chem 234: 2544-2547.

105. Hatzivassiliou G, Zhao F, Bauer DE, Andreadis C, Shaw AN, et al. (2005) ATP citrate lyase inhibition can suppress tumor cell growth. Cancer Cell 8: 311-321. 
106. Tong WH, Rouault TA (2007) Metabolic regulation of citrate and iron by aconitases: role of iron-sulfur cluster biogenesis. Biometals 20: 549-564.

107. Rotig A, de Lonlay P, Chretien D, Foury F, Koenig M, et al. (1997) Aconitase and mitochondrial iron-sulphur protein deficiency in Friedreich ataxia. Nat Genet 17: 215-217.

108. Napoli E, Taroni F, Cortopassi GA (2006) Frataxin, iron-sulfur clusters, heme, ROS, and aging. Antioxid Redox Signal 8: 506-516.

109. Bulteau AL, Lundberg KC, Ikeda-Saito M, Isaya G, Szweda LI (2005) Reversible redox-dependent modulation of mitochondrial aconitase and proteolytic activity during in vivo cardiac ischemia/reperfusion. Proc Natl Acad Sci U S A 102: 59875991.

110. Castro L, Rodriguez M, Radi R (1994) Aconitase is readily inactivated by peroxynitrite, but not by its precursor, nitric oxide. Journal of Biological Chemistry 269: 29409-29415.

111. Zhang S-J, Sandstrom ME, Lanner JT, Thorell A, Westerblad H, et al. (2007) Activation of aconitase in mouse fast-twitch skeletal muscle during contractionmediated oxidative stress. Am J Physiol Cell Physiol 293: C1154-1159.

112. Lin G, Brownsey R, MacLeod K (2009) Regulation of mitochondrial aconitase by phosphorylation in diabetic rat heart. Cellular and Molecular Life Sciences 66: 919-932.

113. Pitula JS, Deck KM, Clarke SL, Anderson SA, Vasanthakumar A, et al. (2004) Selective inhibition of the citrate-to-isocitrate reaction of cytosolic aconitase by phosphomimetic mutation of serine-711. Proc Natl Acad Sci U S A 101: 1090710912.

114. Peters R, Wakelin RW, Buffa P (1953) Biochemistry of Fluoroacetate Poisoning The Isolation and some Properties of the Fluorotricarboxylic Acid Inhibitor of Citrate Metabolism. Proceedings of the Royal Society of London Series B Biological Sciences 140: 497-506.

115. Morrison JF, Peters RA (1954) Biochemistry of fluoroacetate poisoning: the effect of fluorocitrate on purified aconitase. Biochem J 58: 473-479. 
116. Kent TA, Emptage MH, Merkle H, Kennedy MC, Beinert H, et al. (1985)

Mossbauer studies of aconitase. Substrate and inhibitor binding, reaction intermediates, and hyperfine interactions of reduced 3Fe and 4Fe clusters. Journal of Biological Chemistry 260: 6871-6881.

117. Lauble H, Kennedy MC, Emptage MH, Beinert H, Stout CD (1996) The reaction of fluorocitrate with aconitase and the crystal structure of the enzyme-inhibitor complex. Proceedings of the National Academy of Sciences of the United States of America 93: 13699-13703.

118. Kun E, Kirsten E, Sharma ML (1977) Enzymatic formation of glutathione-citryl thioester by a mitochondrial system and its inhibition by (-)erythrofluorocitrate. Proceedings of the National Academy of Sciences 74: 4942-4946.

119. Proudfoot AT BS, and Vale JA (2006) Sodium Fluoroacetate poisoning. Toxicological Reviews 25: 213-219.

120. Goncharov NV, Jenkins RO, Radilov AS (2006) Toxicology of fluoroacetate: a review, with possible directions for therapy research. Journal of Applied Toxicology 26: 148-161.

121. Swanson RA, Graham SH (1994) Fluorocitrate and fluoroacetate effects on astrocyte metabolism in vitro. Brain Research 664: 94-100.

122. B. K. Matheson JLA, J. Zou, R. Patel, R. B. Franklin (2007) Effect of metabolic inhibitors on ATP and citrate content in PC3 prostate cancer cells. The Prostate 67: 1211-1218.

123. Pantopoulos K (2004) Iron Metabolism and the IRE/IRP Regulatory System: An Update. Annals of the New York Academy of Sciences 1012: 1-13.

124. Haile DJ, Rouault TA, Tang CK, Chin J, Harford JB, et al. (1992) Reciprocal control of RNA-binding and aconitase activity in the regulation of the ironresponsive element binding protein: role of the iron-sulfur cluster. Proc Natl Acad Sci U S A 89: 7536-7540.

125. Hentze MW, Caughman SW, Rouault TA, Barriocanal JG, Dancis A, et al. (1987) Identification of the iron-responsive element for the translational regulation of human ferritin mRNA. Science 238: 1570-1573. 
126. Casey JL, Di Jeso B, Rao K, Klausner RD, Harford JB (1988) Two genetic loci participate in the regulation by iron of the gene for the human transferrin receptor. Proc Natl Acad Sci U S A 85: 1787-1791.

127. Recalcati S, Minotti G, Cairo G (2010) Iron Regulatory Proteins: From Molecular Mechanisms to Drug Development. Antioxidants \& Redox Signaling 13: 15931616.

128. LaVaute T, Smith S, Cooperman S, Iwai K, Land W, et al. (2001) Targeted deletion of the gene encoding iron regulatory protein-2 causes misregulation of iron metabolism and neurodegenerative disease in mice. Nat Genet 27: 209-214.

129. Cooperman SS, Meyron-Holtz EG, Olivierre-Wilson H, Ghosh MC, McConnell JP, et al. (2005) Microcytic anemia, erythropoietic protoporphyria, and neurodegeneration in mice with targeted deletion of iron-regulatory protein 2. Blood 106: 1084-1091.

130. Meyron-Holtz EG, Ghosh MC, Iwai K, LaVaute T, Brazzolotto X, et al. (2004) Genetic ablations of iron regulatory proteins 1 and 2 reveal why iron regulatory protein 2 dominates iron homeostasis. EMBO J 23: 386-395.

131. Kim H-Y, LaVaute T, Iwai K, Klausner RD, Rouault TA (1996) Identification of a Conserved and Functional Iron-responsive Element in the 5'-Untranslated Region of Mammalian Mitochondrial Aconitase. J Biol Chem 271: 24226-24230.

132. Schalinske KL, Chen OS, Eisenstein RS (1998) Iron Differentially Stimulates Translation of Mitochondrial Aconitase and Ferritin mRNAs in Mammalian Cells. Journal of Biological Chemistry 273: 3740-3746.

133. Martin RB (1986) Citrate binding of Al3+ and Fe3+. Journal of Inorganic Biochemistry 28: 181-187.

134. Chen XJ, Wang X, Kaufman BA, Butow RA (2005) Aconitase Couples Metabolic Regulation to Mitochondrial DNA Maintenance. Science 307: 714-717.

135. Chen XJ, Wang X, Butow RA (2007) Yeast aconitase binds and provides metabolically coupled protection to mitochondrial DNA. Proceedings of the National Academy of Sciences 104: 13738-13743.

136. Ponka P (1999) Cell biology of heme. Am J Med Sci 318: 241-256. 
137. Andrews NC (2008) Forging a field: the golden age of iron biology. Blood 112: 219-230.

138. Heath CW, Strauss MB, Castle WB (1932) Quantitative aspects of iron deficiency in hypochromic anemia: The Parenteral Administration of Iron. The Journal of Clinical Investigation 11: 1293-1312.

139. Mims MP, Guan Y, Pospisilova D, Priwitzerova M, Indrak K, et al. (2005)

Identification of a human mutation of DMT1 in a patient with microcytic anemia and iron overload. Blood 105: 1337-1342.

140. Raja KB, Pountney DJ, Simpson RJ, Peters TJ (1999) Importance of Anemia and Transferrin Levels in the Regulation of Intestinal Iron Absorption in Hypotransferrinemic Mice. Blood 94: 3185-3192.

141. Trenor CC, Campagna DR, Sellers VM, Andrews NC, Fleming MD (2000) The molecular defect in hypotransferrinemic mice. Blood 96: 1113-1118.

142. Marignani M, Angeletti S, Bordi C, Malagnino F, Mancino C, et al. (1997) Reversal of long-standing iron deficiency anaemia after eradication of Helicobacter pylori infection. Scand J Gastroenterol 32: 617-622.

143. Al-Sheikh M, Mazurier E, Gardie B, Casadevall N, Galacteros F, et al. (2008) A study of 36 unrelated cases with pure erythrocytosis revealed three new mutations in the erythropoietin receptor gene. Haematologica 93: 1072-1075.

144. Ang SO, Chen H, Hirota K, Gordeuk VR, Jelinek J, et al. (2002) Disruption of oxygen homeostasis underlies congenital Chuvash polycythemia. Nat Genet 32: 614-621.

145. Patnaik MM, Tefferi A (2009) The complete evaluation of erythrocytosis: congenital and acquired. Leukemia 23: 834-844.

146. James C, Ugo V, Le Couedic JP, Staerk J, Delhommeau F, et al. (2005) A unique clonal JAK2 mutation leading to constitutive signalling causes polycythaemia vera. Nature 434: 1144-1148.

147. Spivak JL (2002) Polycythemia vera: myths, mechanisms, and management. Blood 100: 4272-4290. 
148. Lengfelder EMD, Merx KMD, Hehlmann RMD (2006) Diagnosis and Therapy of Polycythemia Vera. Seminars in Thrombosis and Hemostasis: 267-275.

149. Elliott MA, Tefferi A (2005) Thrombosis and haemorrhage in polycythaemia vera and essential thrombocythaemia. British Journal of Haematology 128: 275-290.

150. Wernig G, Mercher T, Okabe R, Levine RL, Lee BH, et al. (2006) Expression of Jak2V617F causes a polycythemia vera-like disease with associated myelofibrosis in a murine bone marrow transplant model. Blood 107: 4274-4281.

151. Xing S, Wanting TH, Zhao W, Ma J, Wang S, et al. (2008) Transgenic expression of JAK2V617F causes myeloproliferative disorders in mice. Blood 111: 5109-5117.

152. Tiedt R, Hao-Shen H, Sobas MA, Looser R, Dirnhofer S, et al. (2008) Ratio of mutant JAK2-V617F to wild-type Jak2 determines the MPD phenotypes in transgenic mice. Blood 111: 3931-3940.

153. Akada H, Yan D, Zou H, Fiering S, Hutchison RE, et al. (2010) Conditional expression of heterozygous or homozygous Jak2V617F from its endogenous promoter induces a polycythemia vera-like disease. Blood 115: 3589-3597.

154. Scott LM, Tong W, Levine RL, Scott MA, Beer PA, et al. (2007) JAK2 Exon 12 Mutations in Polycythemia Vera and Idiopathic Erythrocytosis. New England Journal of Medicine 356: 459-468.

155. Green A, Beer P (2010) Somatic Mutations of IDH1 and IDH2 in the Leukemic Transformation of Myeloproliferative Neoplasms. New England Journal of Medicine 362: 369-370.

156. Delhommeau F, Dupont S, Valle VD, James C, Trannoy S, et al. (2009) Mutation in TET2 in Myeloid Cancers. New England Journal of Medicine 360: 2289-2301.

157. Stohlawetz PJ, Dzirlo L, Hergovich N, Lackner E, Mensik C, et al. (2000) Effects of erythropoietin on platelet reactivity and thrombopoiesis in humans. Blood 95: 2983-2989.

158. Tefferi A, Pardanani A (2011) JAK inhibitors in myeloproliferative neoplasms: rationale, current data and perspective. Blood Reviews In Press, Corrected Proof. 
159. Ludwig H, Fritz E, Kotzmann H, Hocker P, Gisslinger H, et al. (1990) Erythropoietin Treatment of Anemia Associated with Multiple Myeloma. New England Journal of Medicine 322: 1693-1699.

160. Goodnough LT, Skikne B, Brugnara C (2000) Erythropoietin, iron, and erythropoiesis. Blood 96: 823-833.

161. Drueke T (2001) Hyporesponsiveness to recombinant human erythropoietin. Nephrology, Dialysis, Transplantation 16: 25-28.

162. Katodritou E, Zervas K, Terpos E, Brugnara C (2008) Use of erythropoiesis stimulating agents and intravenous iron for cancer and treatment-related anaemia: the need for predictors and indicators of effectiveness has not abated. $\mathrm{Br} \mathbf{J}$ Haematol 142: 3-10.

163. Nulton-Persson AC, Szweda LI (2001) Modulation of Mitochondrial Function by Hydrogen Peroxide. Journal of Biological Chemistry 276: 23357-23361.

164. Deck KM, Vasanthakumar A, Anderson SA, Goforth JB, Kennedy MC, et al. (2009) Evidence That Phosphorylation of Iron Regulatory Protein 1 at Serine 138 Destabilizes the [4Fe-4S] Cluster in Cytosolic Aconitase by Enhancing 4Fe-3Fe Cycling. Journal of Biological Chemistry 284: 12701-12709.

165. Arcasoy MO, Jiang X (2005) Co-operative signalling mechanisms required for erythroid precursor expansion in response to erythropoietin and stem cell factor. British Journal of Haematology 130: 121-129.

166. Halupa A, Chohan M, Stickle NH, Beattie BK, Miller BA, et al. (2005) Erythropoietin receptor Y479 couples to ERK1/2 activation via recruitment of phospholipase C[gamma]. Experimental Cell Research 309: 1-11.

167. Klingmüller U (1997) The Role of Tyrosine Phosphorylation in Proliferation and Maturation of Erythroid Progenitor Cells. European Journal of Biochemistry 249: 637-647.

168. Casar B, Arozarena I, Sanz-Moreno V, Pinto A, Agudo-Ibanez L, et al. (2009) Ras Subcellular Localization Defines Extracellular Signal-Regulated Kinase 1 and 2 Substrate Specificity through Distinct Utilization of Scaffold Proteins. Mol Cell Biol 29: 1338-1353. 
169. Teis D, Wunderlich W, Huber LA (2002) Localization of the MP1-MAPK Scaffold Complex to Endosomes Is Mediated by p14 and Required for Signal Transduction. Developmental Cell 3: 803-814.

170. Torii S, Kusakabe M, Yamamoto T, Maekawa M, Nishida E (2004) Sef Is a Spatial Regulator for Ras/MAP Kinase Signaling. Developmental Cell 7: 33-44.

171. Jaaro H, Rubinfeld H, Hanoch T, Seger R (1997) Nuclear translocation of mitogenactivated protein kinase kinase (MEK1) in response to mitogenic stimulation. Proceedings of the National Academy of Sciences of the United States of America 94: 3742-3747.

172. Galli S, Jahn O, Hitt R, Hesse D, Opitz L, et al. (2009) A New Paradigm for MAPK: Structural Interactions of hERK1 with Mitochondria in HeLa Cells. PLoS ONE 4: e7541.

173. Vannucchi AM, Guglielmelli P, Tefferi A (2009) Advances in Understanding and Management of Myeloproliferative Neoplasms. CA: A Cancer Journal for Clinicians 59: 171-191.

174. Isken F, Schulz TJ, Möhlig M, Pfeiffer AFH, Ristow M (2006) Chemical Inhibition of Citrate Metabolism Alters Glucose Metabolism in Mice. Hormone and Metabolic Research: 543-545.

175. Chen K, Liu J, Heck S, Chasis JA, An X, et al. (2009) Resolving the distinct stages in erythroid differentiation based on dynamic changes in membrane protein expression during erythropoiesis. Proceedings of the National Academy of Sciences 106: 17413-17418.

176. Pop R, Shearstone JR, Shen Q, Liu Y, Hallstrom K, et al. (2010) A Key Commitment Step in Erythropoiesis Is Synchronized with the Cell Cycle Clock through Mutual Inhibition between PU.1 and S-Phase Progression. PLoS Biol 8: e1000484.

177. Hardie DG, Hawley SA, Scott JW (2006) AMP-activated protein kinase-development of the energy sensor concept. J Physiol 574: 7-15.

178. Setsukinai K-i, Urano Y, Kakinuma K, Majima HJ, Nagano T (2003) Development of Novel Fluorescence Probes That Can Reliably Detect Reactive Oxygen Species 
and Distinguish Specific Species. Journal of Biological Chemistry 278: 31703175.

179. Poot M, Zhang YZ, Kramer JA, Wells KS, Jones LJ, et al. (1996) Analysis of mitochondrial morphology and function with novel fixable fluorescent stains. $\mathbf{J}$ Histochem Cytochem 44: 1363-1372.

180. Haq R, Halupa A, Beattie BK, Mason JM, Zanke BW, et al. (2002) Regulation of erythropoietin-induced STAT serine phosphorylation by distinct mitogenactivated protein kinases. J Biol Chem 277: 17359-17366.

181. Magocsi M, Apati A, Gati R, Kolonics A (1999) Signalling mechanisms and the role of calcineurin in erythropoiesis. Immunol Lett 68: 187-195.

182. Einar Andreas S, Marit Elise H, Kristine Bjerve G, Guri D, Erlend Bremertun S, et al. (2006) PI3K/Akt-dependent Epo-induced signalling and target genes in human early erythroid progenitor cells. British Journal of Haematology 135: 117-128.

183. Schmidt E, Fichelson S, Feller S (2004) PI3 kinase is important for Ras, MEK and Erk activation of Epo-stimulated human erythroid progenitors. BMC Biology 2: 7.

184. Anjum R, Blenis J (2008) The RSK family of kinases: emerging roles in cellular signalling. Nat Rev Mol Cell Biol 9: 747-758.

185. Stark C, Breitkreutz B-J, Reguly T, Boucher L, Breitkreutz A, et al. (2005) BioGRID: a general repository for interaction datasets. Nucleic Acids Research 34: D535-D539.

186. Obenauer JC, Cantley LC, Yaffe MB (2003) Scansite 2.0: proteome-wide prediction of cell signaling interactions using short sequence motifs. Nucl Acids Res 31: 3635-3641.

187. Philipova R, Whitaker M (2005) Active ERK1 is dimerized in vivo: bisphosphodimers generate peak kinase activity and monophosphodimers maintain basal ERK1 activity. J Cell Sci 118: 5767-5776.

188. Casar B, Pinto A, Crespo P (2008) Essential role of ERK dimers in the activation of cytoplasmic but not nuclear substrates by ERK-scaffold complexes. Mol Cell 31: 708-721. 
189. Quintas-Cardama A, Vaddi K, Liu P, Manshouri T, Li J, et al. (2010) Preclinical characterization of the selective JAK1/2 inhibitor INCB018424: therapeutic implications for the treatment of myeloproliferative neoplasms. Blood 115: 31093117.

190. Zou H, Yan D, Mohi G (2011) Differential biological activity of disease-associated JAK2 mutants. FEBS Letters 585: 1007-1013.

191. Greer JP (2008) Wintrobe's Clinical Hematology. Hagerstown: Lippincott, Williams \& Wilkins. 3232 p.

192. Lyoumi S, Abitbol M, Andrieu V, Henin D, Robert E, et al. (2007) Increased plasma transferrin, altered body iron distribution, and microcytic hypochromic anemia in ferrochelatase-deficient mice. Blood 109: 811-818.

193. Savarie PJ (1984) Toxic characteristics of fluorocitrate, the toxic metabolite of compound 1080. In: D.O. C, editor. Proceedings Eleventh Vertebrate Pest Conference. Lincoln. pp. 132-137.

194. Sankaran VG, Orkin SH, Walkley CR (2008) Rb intrinsically promotes erythropoiesis by coupling cell cycle exit with mitochondrial biogenesis. Genes and Development 22: 463-475.

195. Leiter LM, Thatte HS, Okafor C, Marks PW, Golan DE, et al. (1999) Chloramphenicol-induced mitochondrial dysfunction is associated with decreased transferrin receptor expression and ferritin synthesis in K562 cells and is unrelated to IRE-IRP interactions. J Cell Physiol 180: 334-344.

196. Inoue S-I, Yokota M, Nakada K, Miyoshi H, Hayashi J-I (2007) Pathogenic mitochondrial DNA-induced respiration defects in hematopoietic cells result in anemia by suppressing erythroid differentiation. FEBS Letters 581: 1910-1916.

197. Diwan A, Koesters AG, Odley AM, Pushkaran S, Baines CP, et al. (2007) Unrestrained erythroblast development in Nix-/- mice reveals a mechanism for apoptotic modulation of erythropoiesis. Proceedings of the National Academy of Sciences 104: 6794-6799. 
198. Chen ML, Logan TD, Hochberg ML, Shelat SG, Yu X, et al. (2009) Erythroid dysplasia, megaloblastic anemia, and impaired lymphopoiesis arising from mitochondrial dysfunction. Blood 114: 4045-4053.

199. Alonso M, Melani M, Converso D, Jaitovich A, Paz C, et al. (2004) Mitochondrial extracellular signal-regulated kinases $1 / 2$ (ERK1/2) are modulated during brain development. Journal of Neurochemistry 89: 248-256.

200. Poderoso C, Converso DP, Maloberti P, Duarte A, Neuman I, et al. (2008) A Mitochondrial Kinase Complex Is Essential to Mediate an ERK1/2-Dependent Phosphorylation of a Key Regulatory Protein in Steroid Biosynthesis. PLoS ONE 3: e1443.

201. Wernig G, Gonneville JR, Crowley BJ, Rodrigues MS, Reddy MM, et al. (2008) The Jak2V617F oncogene associated with myeloproliferative diseases requires a functional FERM domain for transformation and for expression of the Myc and Pim proto-oncogenes. Blood 111: 3751-3759.

202. Elagib KE, Xiao M, Hussaini IM, Delehanty LL, Palmer LA, et al. (2004) Jun Blockade of Erythropoiesis: Role for Repression of GATA-1 by HERP2. Molecular and Cellular Biology 24: 7779-7794.

203. Choi Y, Elagib KE, Delehanty LL, Goldfarb AN (2006) Erythroid inhibition by the leukemic fusion AML1-ETO is associated with impaired acetylation of the major erythroid transcription factor GATA-1. Cancer Res 66: 2990-2996.

204. Seger R, Pinto A, Crespo P (2010) Analysis of ERKs’ Dimerization by Electrophoresis. MAP Kinase Signaling Protocols: Humana Press. pp. 335-342. 205. Nobes PR, Carter AB (1990) Reticulocyte counting using flow cytometry. J Clin Pathol 43: 675-678.

206. Deak M, Clifton AD, Lucocq JM, Alessi DR (1998) Mitogen- and stress-activated protein kinase-1 (MSK1) is directly activated by MAPK and SAPK2/p38, and may mediate activation of CREB. EMBO J 17: 4426-4441.

207. Chen RH, Sarnecki C, Blenis J (1992) Nuclear localization and regulation of erkand rsk-encoded protein kinases. Mol Cell Biol 12: 915-927. 
208. Smith JA, Poteet-Smith CE, Malarkey K, Sturgill TW (1999) Identification of an Extracellular Signal-regulated Kinase (ERK) Docking Site in Ribosomal S6 Kinase, a Sequence Critical for Activation by ERK in Vivo. Journal of Biological Chemistry 274: 2893-2898.

209. Smith JA, Poteet-Smith CE, Xu Y, Errington TM, Hecht SM, et al. (2005) Identification of the first specific inhibitor of p90 ribosomal S6 kinase (RSK) reveals an unexpected role for RSK in cancer cell proliferation. Cancer Res 65: 1027-1034.

210. Sapkota GP, Cummings L, Newell FS, Armstrong C, Bain J, et al. (2007) BI-D1870 is a specific inhibitor of the p90 RSK (ribosomal S6 kinase) isoforms in vitro and in vivo. Biochem J 401: 29-38.

211. Poteet-Smith CE, Smith JA, Lannigan DA, Freed TA, Sturgill TW (1999) Generation of Constitutively Active p90 Ribosomal S6 Kinase in Vivo. Journal of Biological Chemistry 274: 22135-22138.

212. Hanlon M, Sturgill TW, Sealy L (2001) ERK2- and p90Rsk2-dependent Pathways Regulate the CCAAT/Enhancer-binding Protein-beta Interaction with Serum Response Factor. Journal of Biological Chemistry 276: 38449-38456.

213. Keerthivasan G, Small S, Liu H, Wickrema A, Crispino JD (2010) Vesicle trafficking plays a novel role in erythroblast enucleation. Blood 116: 3331-3340.

214. Wang X, Flynn A, Waskiewicz AJ, Webb BLJ, Vries RG, et al. (1998) The Phosphorylation of Eukaryotic Initiation Factor eIF4E in Response to Phorbol Esters, Cell Stresses, and Cytokines Is Mediated by Distinct MAP Kinase Pathways. Journal of Biological Chemistry 273: 9373-9377.

215. Ueda T, Watanabe-Fukunaga R, Fukuyama H, Nagata S, Fukunaga R (2004) Mnk2 and Mnk1 Are Essential for Constitutive and Inducible Phosphorylation of Eukaryotic Initiation Factor 4E but Not for Cell Growth or Development. Mol Cell Biol 24: 6539-6549.

216. Knauf U, Tschopp C, Gram H (2001) Negative Regulation of Protein Translation by Mitogen-Activated Protein Kinase-Interacting Kinases 1 and 2. Mol Cell Biol 21: 5500-5511. 
217. Shaw PE, Saxton J (2003) Ternary complex factors: prime nuclear targets for mitogen-activated protein kinases. The International Journal of Biochemistry \& Cell Biology 35: 1210-1226.

218. Shipley J, Sheer D, Dalton S, Treisman R, Patel K (1994) Mapping of the Human SAP1 (SRF Accessory Protein 1) Gene and SAP2, a Gene Encoding a Related Protein, to Chromosomal Bands 1q32 and 12q23, Respectively. Genomics 23: 710-711.

219. Haagenson KK, Wu GS (2010) Mitogen activated protein kinase phosphatases and cancer. Cancer Biology \& Therapy 9: 337-340.

220. Owens DM, Keyse SM (2007) Differential regulation of MAP kinase signalling by dual-specificity protein phosphatases. Oncogene 26: 3203-3213.

221. Alonso A, Sasin J, Bottini N, Friedberg I, Friedberg I, et al. (2004) Protein Tyrosine Phosphatases in the Human Genome. Cell 117: 699-711.

222. Janssens V, Goris J (2001) Protein phosphatase 2A: a highly regulated family of serine/threonine phosphatases implicated in cell growth and signalling. Biochem $\mathrm{J}$ 353: 417-439.

223. Muda M, Theodosiou A, Rodrigues N, Boschert U, Camps M, et al. (1996) The Dual Specificity Phosphatases M3/6 and MKP-3 Are Highly Selective for Inactivation of Distinct Mitogen-activated Protein Kinases. Journal of Biological Chemistry 271: 27205-27208.

224. Marchetti S, Gimond C, Chambard J-C, Touboul T, Roux D, et al. (2005) Extracellular Signal-Regulated Kinases Phosphorylate Mitogen-Activated Protein Kinase Phosphatase 3/DUSP6 at Serines 159 and 197, Two Sites Critical for Its Proteasomal Degradation. Mol Cell Biol 25: 854-864.

225. Abraham D, Podar K, Pacher M, Kubicek M, Welzel N, et al. (2000) Raf-1associated Protein Phosphatase 2A as a Positive Regulator of Kinase Activation. Journal of Biological Chemistry 275: 22300-22304.

226. Alessi DR, Gomez N, Moorhead G, Lewis T, Keyse SM, et al. (1995) Inactivation of p42 MAP kinase by protein phosphatase 2A and a protein tyrosine phosphatase, but not CL100, in various cell lines. Current Biology 5: 283-295. 
227. Sontag E, Fedorov S, Kamibayashi C, Robbins D, Cobb M, et al. (1993) The interaction of SV40 small tumor antigen with protein phosphatase 2A stimulates the map kinase pathway and induces cell proliferation. Cell 75: 887-897.

228. Favre B, Zolnierowicz S, Turowski P, Hemmings BA (1994) The catalytic subunit of protein phosphatase $2 \mathrm{~A}$ is carboxyl-methylated in vivo. Journal of Biological Chemistry 269: 16311-16317.

229. Palanivel R, Veluthakal R, Kowluru A (2004) Regulation by glucose and calcium of the carboxylmethylation of the catalytic subunit of protein phosphatase $2 \mathrm{~A}$ in insulin-secreting INS-1 cells. American Journal of Physiology - Endocrinology And Metabolism 286: E1032-E1041.

230. Sjoholm A, Lehtihet M, Efanov AM, Zaitsev SV, Berggren P-O, et al. (2002) Glucose Metabolites Inhibit Protein Phosphatases and Directly Promote Insulin Exocytosis in Pancreatic $\mathbf{I}^{2}$-Cells. Endocrinology 143: 4592-4598.

231. Seamon K, Daly JW (1981) Activation of adenylate cyclase by the diterpene forskolin does not require the guanine nucleotide regulatory protein. Journal of Biological Chemistry 256: 9799-9801.

232. Neviani P, Santhanam R, Trotta R, Notari M, Blaser BW, et al. (2005) The tumor suppressor PP2A is functionally inactivated in blast crisis CML through the inhibitory activity of the BCR/ABL-regulated SET protein. Cancer Cell 8: 355368.

233. Fernandez JJ, Candenas ML, Souto ML, Trujillo MM, Norte M (2002) Okadaic acid, useful tool for studying cellular processes. Curr Med Chem 9: 229-262.

234. Nekrasova T, Shive C, Gao Y, Kawamura K, Guardia R, et al. (2005) ERK1deficient mice show normal $\mathrm{T}$ cell effector function and are highly susceptible to experimental autoimmune encephalomyelitis. J Immunol 175: 2374-2380.

235. Pages G, Guein S, Grall D, Bonino F, Smith A, et al. (1999) Defective Thymocyte Maturation in p44 MAP Kinase (Erk 1) Knockout Mice. Science 286: 1374-1377. 236. Saba-El-Leil MK, Vella FD, Vernay B, Voisin L, Chen L, et al. (2003) An essential function of the mitogen-activated protein kinase Erk2 in mouse trophoblast development. EMBO Rep 4: 964-968. 
237. Kerenyi MA, Grebien F, Gehart H, Schifrer M, Artaker M, et al. (2008) Stat5 regulates cellular iron uptake of erythroid cells via IRP-2 and TfR-1. Blood 112: 3878-3888.

238. Tanno T, Noh S-J, Byrnes C, Bhupatiraju A, Meier ER, et al. (2008) Iron Depleted Erythropoiesis: Slow but Effective. ASH Annual Meeting Abstracts 112: 418-.

239. Boas FE, Forman L, Beutler E (1998) Phosphatidylserine exposure and red cell viability in red cell aging and in hemolytic anemia. Proc Natl Acad Sci U S A 95: 3077-3081.

240. Wellen KE, Hatzivassiliou G, Sachdeva UM, Bui TV, Cross JR, et al. (2009) ATPCitrate Lyase Links Cellular Metabolism to Histone Acetylation. Science 324: 1076-1080.

241. Dang L, White DW, Gross S, Bennett BD, Bittinger MA, et al. (2009) Cancerassociated IDH1 mutations produce 2-hydroxyglutarate. Nature 462: 739-744.

242. Struys EA, Salomons GS, Achouri Y, Van Schaftingen E, Grosso S, et al. (2005) Mutations in the d-2-Hydroxyglutarate Dehydrogenase Gene Cause d-2Hydroxyglutaric Aciduria. The American Journal of Human Genetics 76: 358360.

243. Aghili M, Zahedi F, Rafiee E (2009) Hydroxyglutaric aciduria and malignant brain tumor: a case report and literature review. Journal of Neuro-Oncology 91: 233236.

244. Dalby KN, Morrice N, Caudwell FB, Avruch J, Cohen P (1998) Identification of Regulatory Phosphorylation Sites in Mitogen-activated Protein Kinase (MAPK)activated Protein Kinase-1a/p90 rsk That Are Inducible by MAPK. Journal of Biological Chemistry 273: 1496-1505.

245. Roux PP, Richards SA, Blenis J (2003) Phosphorylation of p90 Ribosomal S6 Kinase (RSK) Regulates Extracellular Signal-Regulated Kinase Docking and RSK Activity. Molecular and Cellular Biology 23: 4796-4804.

246. McElveen RL, Lou TF, Reese K, Xia S, Baliga BS, et al. (2005) Erk pathway inhibitor U0126 induces gamma-globin expression in erythroid cells. Cell Mol Biol (Noisy-le-grand) 51: 215-227. 
247. Zhang F, Strand A, Robbins D, Cobb MH, Goldsmith EJ (1994) Atomic structure of the MAP kinase ERK2 at 2.3 A resolution. Nature 367: 704-711.

248. Olsen JV, Blagoev B, Gnad F, Macek B, Kumar C, et al. (2006) Global, In Vivo, and Site-Specific Phosphorylation Dynamics in Signaling Networks. Cell 127: 635-648.

249. Subramanian A, Tamayo P, Mootha VK, Mukherjee S, Ebert BL, et al. (2005) Gene set enrichment analysis: A knowledge-based approach for interpreting genomewide expression profiles. Proceedings of the National Academy of Sciences of the United States of America 102: 15545-15550.

250. Clark DE, Errington TM, Smith JA, Frierson HF, Weber MJ, et al. (2005) The Serine/Threonine Protein Kinase, p90 Ribosomal S6 Kinase, Is an Important Regulator of Prostate Cancer Cell Proliferation. Cancer Research 65: 3108-3116.

251. Palmer A, Gavin A-C, Nebreda AR (1998) A link between MAP kinase and p34cdc2/cyclin B during oocyte maturation: p90rsk phosphorylates and inactivates the p34cdc2 inhibitory kinase Myt1. EMBO J 17: 5037-5047.

252. Bonni A, Brunet A, West AE, Datta SR, Takasu MA, et al. (1999) Cell Survival Promoted by the Ras-MAPK Signaling Pathway by Transcription-Dependent and -Independent Mechanisms. Science 286: 1358-1362.

253. Anjum R, Roux PP, Ballif BA, Gygi SP, Blenis J (2005) The Tumor Suppressor DAP Kinase Is a Target of RSK-Mediated Survival Signaling. Current Biology 15: 1762-1767.

254. Kim K-W, Kim S-H, Lee E-Y, Kim ND, Kang H-S, et al. (2001) Extracellular Signal-regulated Kinase/90-kDa Ribosomal S6 Kinase/Nuclear Factor- $\hat{I}^{\circ} \mathrm{B}$ Pathway Mediates Phorbol 12-Myristate 13-Acetate-induced Megakaryocytic Differentiation of K562 Cells. Journal of Biological Chemistry 276: 1318613191.

255. Kang S, Dong S, Gu T-L, Guo A, Cohen MS, et al. (2007) FGFR3 Activates RSK2 to Mediate Hematopoietic Transformation through Tyrosine Phosphorylation of RSK2 and Activation of the MEK/ERK Pathway. Cancer Cell 12: 201-214. 
256. Elf S, Blevins D, Jin L, Chung T-W, Williams IR, et al. (2011) p90RSK2 is essential for FLT3-ITD- but dispensable for BCR-ABL-induced myeloid leukemia. Blood 117: 6885-6894.

257. Bain J, Plater L, Elliott M, Shpiro N, Hastie CJ, et al. (2007) The selectivity of protein kinase inhibitors: a further update. Biochem J 408: 297-315.

258. Kuang E, Tang Q, Maul GG, Zhu F (2008) Activation of p90 Ribosomal S6 Kinase by ORF45 of Kaposi's Sarcoma-Associated Herpesvirus and Its Role in Viral Lytic Replication. J Virol 82: 1838-1850.

259. Chen S, MacKintosh C (2009) Differential regulation of NHE1 phosphorylation and glucose uptake by inhibitors of the ERK pathway and p90RSK in 3T3-L1 adipocytes. Cellular Signalling 21: 1984-1993.

260. Fonseca BD, Alain T, Finestone LK, Huang BPH, Rolfe M, et al. (2011)

Pharmacological and Genetic Evaluation of Proposed Roles of Mitogen-activated Protein Kinase/Extracellular Signal-regulated Kinase Kinase (MEK), Extracellular Signal-regulated Kinase (ERK), and p90RSK in the Control of mTORC1 Protein Signaling by Phorbol Esters. Journal of Biological Chemistry 286: 27111-27122.

261. Takaki T, Trenz K, Costanzo V, Petronczki M (2008) Polo-like kinase 1 reaches beyond mitosis--cytokinesis, DNA damage response, and development. Current Opinion in Cell Biology 20: 650-660.

262. Nawijn MC, Alendar A, Berns A (2011) For better or for worse: the role of Pim oncogenes in tumorigenesis. Nat Rev Cancer 11: 23-34.

263. Lowry B, Miller JR, Fraser FC (1971) A new dominant gene mental retardation syndrome. Association with small stature, tapering fingers, characteristic facies, and possible hydrocephalus. Am J Dis Child 121: 496-500.

264. Marques Pereira P, Schneider A, Pannetier S, Heron D, Hanauer A (2010) CoffinLowry syndrome. Eur J Hum Genet 18: 627-633.

265. Dufresne SD, Bjorbak C, El-Haschimi K, Zhao Y, Aschenbach WG, et al. (2001) Altered Extracellular Signal-Regulated Kinase Signaling and Glycogen 
Metabolism in Skeletal Muscle from p90 Ribosomal S6 Kinase 2 Knockout Mice. Mol Cell Biol 21: 81-87.

266. Yang X, Matsuda K, Bialek P, Jacquot S, Masuoka HC, et al. (2004) ATF4 Is a Substrate of RSK2 and an Essential Regulator of Osteoblast Biology: Implication for Coffin-Lowry Syndrome. Cell 117: 387-398.

267. Dumont J, Umbhauer M, Rassinier P, Hanauer A, Verlhac M-H (2005) p90Rsk is not involved in cytostatic factor arrest in mouse oocytes. The Journal of Cell Biology 169: 227-231.

268. Gille H, Kortenjann M, Thomae O, Moomaw C, Slaughter C, et al. (1995) ERK phosphorylation potentiates Elk-1-mediated ternary complex formation and transactivation. EMBO J 14: 951-962.

269. Buchwalter G, Gross C, Wasylyk B (2004) Ets ternary complex transcription factors. Gene 324: 1-14.

270. Cavigelli M, Dolfi F, Claret FX, Karin M (1995) Induction of c-fos expression through JNK-mediated TCF/Elk-1 phosphorylation. EMBO J 14: 5957-5964.

271. Price MA, Cruzalegui FH, Treisman R (1996) The p38 and ERK MAP kinase pathways cooperate to activate Ternary Complex Factors and c-fos transcription in response to UV light. EMBO J 15: 6552-6563.

272. Tian J, Karin M (1999) Stimulation of Elk1 Transcriptional Activity by Mitogenactivated Protein Kinases Is Negatively Regulated by Protein Phosphatase 2B (Calcineurin). Journal of Biological Chemistry 274: 15173-15180.

273. van den Oudenrijn S, von dem Borne AEGK, de Haas M (2000) Differences in megakaryocyte expansion potential between CD34+ stem cells derived from cord blood, peripheral blood, and bone marrow from adults and children. Experimental Hematology 28: 1054-1061.

274. Michejda M (2004) Which stem cells should be used for transplantation? Fetal Diagn Ther 19: 2-8.

275. Grammer TC, Blenis J (1997) Evidence for MEK-independent pathways regulating the prolonged activation of the ERK-MAP kinases. Oncogene 14: 1635-1642. 
276. Aksamitiene E, Kholodenko BN, Kolch W, Hoek JB, Kiyatkin A (2010) PI3K/Aktsensitive MEK-independent compensatory circuit of ERK activation in ERpositive PI3K-mutant T47D breast cancer cells. Cellular Signalling 22: 13691378.

277. Bapat S, Verkleij A, Post JA (2001) Peroxynitrite activates mitogen-activated protein kinase (MAPK) via a MEK-independent pathway: a role for protein kinase C. FEBS Letters 499: 21-26.

278. Kamata H, Honda S-i, Maeda S, Chang L, Hirata H, et al. (2005) Reactive Oxygen Species Promote TNF[alpha]-Induced Death and Sustained JNK Activation by Inhibiting MAP Kinase Phosphatases. Cell 120: 649-661.

279. Ho Y, Gruhler A, Heilbut A, Bader GD, Moore L, et al. (2002) Systematic identification of protein complexes in Saccharomyces cerevisiae by mass spectrometry. Nature 415: 180-183.

280. Murphy LO, Smith S, Chen R-H, Fingar DC, Blenis J (2002) Molecular interpretation of ERK signal duration by immediate early gene products. Nat Cell Biol 4: 556-564.

281. Wunderlich W, Fialka I, Teis D, Alpi A, Pfeifer A, et al. (2001) A Novel 14Kilodalton Protein Interacts with the Mitogen-Activated Protein Kinase Scaffold Mp1 on a Late Endosomal/Lysosomal Compartment. The Journal of Cell Biology 152: 765-776.

282. Nada S, Hondo A, Kasai A, Koike M, Saito K, et al. (2009) The novel lipid raft adaptor p18 controls endosome dynamics by anchoring the MEK-ERK pathway to late endosomes. EMBO J 28: 477-489.

283. DeFea KA, Zalevsky J, Thoma MS, DÃ@ry O, Mullins RD, et al. (2000) BArrestin-Dependent Endocytosis of Proteinase-Activated Receptor 2 Is Required for Intracellular Targeting of Activated Erk1/2. The Journal of Cell Biology 148: 1267-1282.

284. Tohgo A, Choy EW, Gesty-Palmer D, Pierce KL, Laporte S, et al. (2003) The Stability of the G Protein-coupled Receptor- $\hat{\mathrm{I}}^{2}$-Arrestin Interaction Determines the 
Mechanism and Functional Consequence of ERK Activation. Journal of Biological Chemistry 278: 6258-6267.

285. Vaidyanathan H, Opoku-Ansah J, Pastorino S, Renganathan H, Matter ML, et al. (2007) ERK MAP kinase is targeted to RSK2 by the phosphoprotein PEA-15. Proceedings of the National Academy of Sciences 104: 19837-19842.

286. Pastorino S, Renganathan H, Caliva MJ, Filbert EL, Opoku-Ansah J, et al. (2010) The death effector domain protein PEA-15 negatively regulates T-cell receptor signaling. The FASEB Journal 24: 2818-2828.

287. Dagda RK, Zhu J, Kulich SM, Chu CT (2008) Mitochondrially localized ERK2 regulates mitophagy and autophagic cell stress: implications for Parkinson's disease. Autophagy 4: 770-782.

288. Baines CP, Zhang J, Wang G-W, Zheng Y-T, Xiu JX, et al. (2002) Mitochondrial PKC $\{$ epsilon $\}$ and MAPK Form Signaling Modules in the Murine Heart: Enhanced Mitochondrial PKC \{epsilon\}-MAPK Interactions and Differential MAPK Activation in PKC \{epsilon\}-Induced Cardioprotection. Circ Res 90: 390397.

289. Wang H-G, Rapp UR, Reed JC (1996) Bcl-2 Targets the Protein Kinase Raf-1 to Mitochondria. Cell 87: 629-638.

290. Galmiche A, Fueller J, Santel A, Krohne G, Wittig I, et al. (2008) Isoform-specific Interaction of C-RAF with Mitochondria. Journal of Biological Chemistry 283: 14857-14866.

291. Leinweber BD, Leavis PC, Grabarek Z, Wang CL, Morgan KG (1999) Extracellular regulated kinase $(\mathrm{ERK})$ interaction with actin and the calponin homology $(\mathrm{CH})$ domain of actin-binding proteins. Biochem J 344 Pt 1: 117-123.

292. Ren JG, Li Z, Sacks DB (2007) IQGAP1 modulates activation of B-Raf. Proc Natl Acad Sci U S A 104: 10465-10469.

293. Harrison RE, Sikorski BA, Jongstra J (2004) Leukocyte-specific protein 1 targets the ERK/MAP kinase scaffold protein KSR and MEK1 and ERK2 to the actin cytoskeleton. Journal of Cell Science 117: 2151-2157. 
294. Smith ER, Smedberg JL, Rula ME, Xu X-X (2004) Regulation of Ras-MAPK pathway mitogenic activity by restricting nuclear entry of activated MAPK in endoderm differentiation of embryonic carcinoma and stem cells. The Journal of Cell Biology 164: 689-699.

295. Krogan NJ, Cagney G, Yu H, Zhong G, Guo X, et al. (2006) Global landscape of protein complexes in the yeast Saccharomyces cerevisiae. Nature 440: 637-643.

296. Frémin C, Ezan F, Boisselier P, Bessard A, Pagès G, et al. (2007) ERK2 but not ERK1 plays a key role in hepatocyte replication: An RNAi-mediated ERK2 knockdown approach in wild-type and ERK1 null hepatocytes. Hepatology 45: 1035-1045.

297. Bost F, Aouadi M, Caron L, Even P, Belmonte N, et al. (2005) The Extracellular Signal-Regulated Kinase Isoform ERK1 Is Specifically Required for In Vitro and In Vivo Adipogenesis. Diabetes 54: 402-411.

298. Vantaggiato C, Formentini I, Bondanza A, Bonini C, Naldini L, et al. (2006) ERK1 and ERK2 mitogen-activated protein kinases affect Ras-dependent cell signaling differentially. Journal of Biology 5: 14.

299. Harman D (1956) Aging: a theory based on free radical and radiation chemistry. J Gerontol 11: 298-300.

300. Guralnik JM, Eisenstaedt RS, Ferrucci L, Klein HG, Woodman RC (2004) Prevalence of anemia in persons 65 years and older in the United States: evidence for a high rate of unexplained anemia. Blood 104: 2263-2268.

301. Nilsson-Ehle H, Jagenburg R, Landahl S, Svanborg A, Westin J (1988)

Haematological abnormalities and reference intervals in the elderly. A crosssectional comparative study of three urban Swedish population samples aged 70, 75 and 81 years. Acta Med Scand 224: 595-604.

302. Tettamanti M, Lucca U, Gandini F, Recchia A, Mosconi P, et al. (2010) Prevalence, incidence and types of mild anemia in the elderly: the "Health and Anemia" population-based study. Haematologica 95: 1849-1856. 
303. Chaves PH, Xue QL, Guralnik JM, Ferrucci L, Volpato S, et al. (2004) What constitutes normal hemoglobin concentration in community-dwelling disabled older women? J Am Geriatr Soc 52: 1811-1816.

304. Tefferi A, Vardiman JW (2009) Myelodysplastic Syndromes. New England Journal of Medicine 361: 1872-1885.

305. Yunis AA, Smith US, Restrepo A (1970) Reversible bone marrow suppression from chloramphenicol. A consequence of mitochondrial injury. Arch Intern Med 126: 272-275.

306. Dawson MA, Davis A, Elliott P, Cole-Sinclair M (2005) Linezolid-induced dyserythropoiesis: chloramphenicol toxicity revisited. Intern Med J 35: 626-628.

307. Wulfert M, Küpper AC, Tapprich C, Bottomley SS, Bowen D, et al. (2008) Analysis of mitochondrial DNA in 104 patients with myelodysplastic syndromes. Experimental Hematology 36: 577-586.

308. Schildgen V, Wulfert M, Gattermann N (2011) Impaired mitochondrial gene transcription in myelodysplastic syndromes and acute myeloid leukemia with myelodysplasia-related changes. Experimental Hematology 39: 666-675.e661. 